\title{
B-cell antibody class switchings are pressuromodulated events: Part II, gene recombination
}

\author{
Hemant Sarin
}

\begin{abstract}
Background: The esebssiwaago $T_{\mathrm{Q}}$ method is applicable for the of study cell gene recombination events as the esebssiwaago $T_{\mathrm{Q}}$ is a measure of the intracellular pressure required to establish a horizontal reading frame for alignment of a gene and its intergene bases for maximal transcription and recombination enzyme activity. B-cell differentiation stages have recently been studied by gene esebssiwaago $T_{\mathrm{Q}}$-based pressuromodulation mapping. In this study, the B-cell differentiation stage pressuromodulation map is utilized as a template to simulate B-cell immunoglobulin locus recombination events that take place in the pressuromodulated state in vivo.

Methods: Chromosome 14 (-) strand location 105,566,277 and 106,879,844 germline genes were recombined after determination of gene esebssiwaago $T_{\mathrm{Q}} \mathrm{S}$ with respect to the germline, and then recombined genes were recombined further after determination of gene esebssiwaago $T_{\mathrm{Q}} \mathrm{S}$ with respect to rearranged configurations. For both alleles, first, IGHD_-_ to IGHJ_ was performed, and then IGHV_-_ to IGHD_-_-IGHJ_ was performed. For Allele 1 (IGHM), internal consensus recognition sequence (iCSR) and further CSR isotype switchings were performed; and for Allele 2 (IGHD), homologous recombination was performed and initial allelic exclusion determined.
\end{abstract}

Results: First, the esebssiwaagot $T_{Q}$ of a joining $\left(J_{-}\right)$and diversity $\left(D_{-}{ }_{-}\right)$gene in its native germline configuration is the basis for predictable subsequent gene rearrangement. Second, $D_{-}-{ }_{-}$to $J_{-}$gene recombination events are biallelic and mutually exclusive. Third, the entire process from beginning to end depends on the grade of the pressuromodulation effect, and as per the classical pathway it is an antigen presenting cell (APC)-dependent CD4R+ T-cell-mediated B-cell polarization process. Fourth, CD4R+ T-cells are positively pressuromodulated, while B-cells are subject to the effect of both positive and negative forms of antigen pressuromodulation. And fifth, B-cell to plasma cell transformation and the extra-nodal periphery/tissue nidus phase take place in the presence of antigen load and either positive or negative pressuromodulation of the cell to its recombined antibody gene expression intracellular pressure.

Conclusions: B-cell gene recombination rearrangement events can be predicted with a reasonable degree of certainty. It is envisioned that further esebssiwaago $T_{\mathrm{Q}}$-based study of the remaining B-cell variability gene recombinations isotype switching events will further our understanding of pressuromodulated basis for antigen selection including the evolutionary underpinnings of.

Keywords: Horizontal alignment, esebssiwaagoT, Pressurotopic, Anisotropy, Mesotropy, Stabilizing isotropy, Supra-pressuromodulated gene, Infra-pressuromodulated gene, Pressuromodulator, Cell polarization, V(D)J gene rearrangement, Internal, Consensus sequence recognition, Homologous recombination, Initial allelic exclusion, Immunoglobulin, Classical pathway, Non-classical pathway

Correspondence: hsmd74@hotmail.com

Freelance Investigator in Translational Science and Medicine (unaffiliated),

833 Carroll Road, Charleston, West Virginia, USA 


\section{Background}

Gene transcription is a pressuromodulated process, pressurotopic [1-3]. B-cell maturation has recently been studied by gene esebssiwaago $T_{\mathrm{Q}}$-based pressuromodulation mapping of B-cell differentiation stage markers [4]. The esebssiwaago $T_{\mathrm{Q}}$-based pressuromodulation map of B-cell differentiation stage genes accurately simulates Bcell maturation in vivo and applies to both the classical B-cell maturation pathway (2-allele T-cell mediated pressuromodulation effect pathway) and the parallel alternative non-classical B-cell maturation pathway (1-allele Tcell independent antigen-mediated pressuromodulation effect pathway).

The classical 2-allele T-cell dependent B-cell maturation pathway involves three phases.

The first phase is the antigen presenting cell (APC)more primed CD4R+ CD40LG T-cell-mediated CD40R Bcell cell membrane $(\mathrm{CM})$ polarization effect or less primed T-cell-mediated polarization effect myeloid bone marrow phase until the Immature B-cell stage (CM IgM+), during which there is Allele 1 (IGHM) VDJ and internal consensus sequence recognition (iCSR) (CM IgM+) and after which there is Allele 2 (IGHD) VDJ.

The second phase is the CD4R+ CD40LG T-cellmediated CD40R B-cell polarization effect lymph node phase until either the Mature naïve B-cell (CM IgM+ $\operatorname{IgD}+$ or $\operatorname{Ig} \mathrm{M}+\operatorname{Ig} \mathrm{M}+$ ) or Evolved mature naïve $\mathrm{B}$-cell $(\mathrm{CM}$ IgG_ + IgG_+) stage, during which there is Allele 2 (IGHD) post-V(D)J homologous recombination (HR) or initial allelic exclusion followed by delayed IgM iCSR and primary, secondary or further CSR isotype switching [5].

And, the third phase is the T-cell independent B-cell $\mathrm{CM}$ receptor antigenic pressuromodulation effectmediated extra-nodal periphery/tissue nidus secretory antibody phase upon exposure to either positive pressuromodulator antigens or negative pressuromodulator antigens, during which there is transformation from B-cell to B-pre-plasma cell followed by B-plasma cell node exiting diapedesis as a secretory long-lived B-plasma cell/plasmablast $[6,7]$.

The parallel alternate non-classical 1-allele T-cell independent B-cell maturation pathway involves two phases, the myeloid bone marrow phase during acute exposure to antigenic positve pressuromodulators, when the T-cell is out of intracellular pressure window for CD4OLG expression, making the myeloid phase a $\mathrm{T}$-cell independent process; and then the extra-nodal periphery/tissue nidus secretory anibody B-plasma cell phase, which is also a Tcell independent pressuromodulator antigen dependent process.

The non-classical pathway myeloid bone marrow phase involves B-cell CM toll-like receptor (TLR)-mediated endocytosis for example, which substitutes for the
T-cell-mediated B-cell polarization pressuromodulation effect in oscillating B-cell intracellular pressure in the marrow [ $\subset 0.26$ esebssiwaago $T_{\mathrm{Q}}$ units $(C D 40) \quad 0.36-$ 0.41 esebssiwaago $T_{\mathrm{Q}}$ units (PRDM1-0.41) 0.10-0.14 esebssiwaago $T_{\mathrm{Q}}$ units $\supset$ ] [4] through the Mature naïve B-cell stage (CM IgM+ IgD-). Thus, during the marrow phase, there is Allele 1 (IGHM) VDJ, iCSR (CM IgM+) and Allele 2 (IGHD) VDJ, which results in a circulating Allele 1 IgM+ only Mature (naïve) B-cell [8].

And, the non-classical pathway T-cell independent extra-marrow periphery/tissue nidus phase results in the IgM+ only (IgM+ IgD-) secretory B-plasma cell or in a further CSRing B-cell and a Ig_+ only (Ig_+ IgD-) secretory B-plasma cell, both short-lived B-plasma cells/ plasmablasts $[6,9]$.

The general intervals of B-cell gene recombination events are shown on the recently developed B-cell differentiation stage pressuromodulation map [4], as are general intervals of internal consensus sequence recognition (iCSR), homologous recombination and further CSR antibody isotype switchings. Thus, the B-cell differentiation stage pressuromodulation map serves as a template for predicting B-cell gene rearrangement events.

B-cell gene recombination events can be predicted for both B-cell differentiation pathways when the following points are considered:

(1) The discordant mechanisms of the recombination enzyme actions on DNA segments, (a) RAG1 and RAG2 recombinases facilitate the excision of intervening variability $\left(V_{-}-{ }_{-}\right)$, diversity $\left(D_{-}{ }_{-}\right)$and joining $\left(J_{-}\right)$genes, where $\mathrm{V}(\mathrm{D}) \mathrm{J}$ recombination does not require an esebssiwaago $T_{\mathrm{Q}}$ match [4] because the mechanism is as such [10], while (b) AICDA and APOBEC3A-G cytidine deaminases facilitate the excision of intervening heavy chain $\left(I_{G} H_{-}\right)$genes, where iCSR [10, 11], CSR recombination [10, 12, 13] and B-cell homologous recombination [14] do require an esebssiwaago $T_{\mathrm{Q}}$ match [4];

(2) B-cell gene recombination begins at greater intracellular pressure on Allele 1 (IGHM) and results in earlier assembly and presentation of IgM on the cell membrane (CM) as compared to Allele 2 (IGHD);

(3) $D_{-}$-_ to $J_{-}$gene rearrangement process of the VDJ $[10,15-17]$ is a limited step process for each allele and the number of steps depend on the grade of the positive antigen pressuromodulation effect;

(4) non-functional $D_{-}$-_ genes are not present in the VDJ [18], as they serve only the purpose of being stepping stone recombination genes for 2-step $D_{-}$to $J_{-}$, and include IGHD1-20 (nf), IGHD4-11 (nf) and IGHD5-18 ( $n f)$ with the exception of IGHD7$27(n f)$, which does not participate; and 
(5) the percentage of V(D)J IGHJ_ genes [18] for Allele 1 (IGHM) 2-step $D_{--}$- to $J_{-}$is $40 \%$ (IGHJ6) and Allele 1 (IGHM) 1 -step $D_{--}$to $J_{-}$is $10 \%$ (IGHJ5), and for Allele 2 (IGHD) 2-step $D_{--}$to $J_{-}$is $32 \%$ (IGHJ4) and Allele 2 (IGHD) 1-step $D_{-}-$to $J_{-}$is $8.5 \%$ (IGHJ3), 1.5\% (IGHJ2) and 8.5\% (IGHJ1).

In this study, B-cell gene recombination is studied by determining germline gene esebssiwaago $T_{\mathrm{Q}} \mathrm{S}$, and rearranging germline genes to simulate actual pressuromodulated in vivo gene recombination events including (1) $I G H D_{-}$- to $I G H J_{-}$, (2) IGHV - to IGHD_-_-IGHJ_, (3) internal consensus sequence recognition (iCSR) for Allele 1 (IGHM) and homologous recombination or initial allelic exclusion for Allele 2 (IGHD); and (4) further CSR isotype switchings for Allele 1 (IGHM) or for both alleles.

\section{Methods}

\section{Data mining}

Locations of germline Ig heavy chain locus genes between chromosome $14(-)$ strand location 105,566,277 and 106,879,844 [19], as well as locations of downstream and upstream genes were mined at GeneCards (https:// www.genecards.org/) genomic neighborhood GeneLoc genome locator database and at LNCipedia.org database (http://www.lncipedia.org/), pseudogenes included and enhancers excluded [2, 4] (Additional file 1: Table S1).

The $5^{\prime}$ - > 3' direction episodic sub-episode sums split-integrated weighted average-averaged gene overexpression tropy quotient (esebssiwaago $T_{\mathrm{Q}}$ ) method and overall approach to gene rearrangement

The downstream and upstream intergene base distances were tabulated, and then the final $5^{\prime}->3^{\prime}$ esebssiwaago $T_{\mathrm{Q}}$ (fract) for each gene was calculated in upstream anisotropic, upstream mesotropic, downstream anisotropic and downstream mesotropic parts. First, the $3^{\prime}->5^{\prime}$ and $5^{\prime}->3^{\prime}$ direction paired point tropy quotients $\left(\operatorname{prp} T_{\mathrm{Q}}\right.$; fract) were determined. Second, initial anisotropic and mesotropic subepisode blocks (SEB; ASEB, MSEB) were determined, which are constant per episode where the number of initial SEBs for establishing a gene category with $100 \%$ sensitivity and $100 \%$ specificity (100\% accuracy) are 5 initial SEBs for an Episode 2 category gene, 7 initial SEBs for an Episode 3 category gene, 9 initial SEBs for an Episode 4 gene, 11 initial SEBs for an Episode 5 gene, 13 initial SEBs for an Episode 6 gene. Third, on the basis of the initial SEBs, the final anisotropic and mesotropic sub-episode blocks (SEB; ASEB, MSEB) were determined, which are variable. And fourth, the $5^{\prime}->3^{\prime}$ direction esebssiwaago $T_{\mathrm{Q}} \mathrm{S}$ to the final esebssiwaa$g o T_{\mathrm{Q}}$ were determined, where a gene with an anisotropic final esebssiwaago $T_{\mathrm{Q}}$ for $<0.25$ is an infrapressuromodulated gene (Infra gene), and where a gene with a mesotropic final esebssiwaago $T_{\mathrm{Q}} \geq 0.25<0.75$ is a supra-pressuromodulated gene (Supra gene). The detailed esebssiwaago $T_{\mathrm{Q}}$ method is in references $[2,4]$.

Germline genes were recombined after determination of gene esebssiwaago $T_{\mathrm{Q}} \mathrm{S}$ with respect to the germline, and recombined genes were recombined further after determination of gene esebssiwaago $T_{\mathrm{Q}} \mathrm{S}$ with respect to the rearranged configuration. For both alleles, first, $I G H D_{-}$- $_{\text {- }}$ to $I G H J_{-}$recombination was performed, and

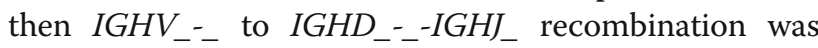
performed. For Allele 1 (IGHM), internal consensus sequence recognition (iCSR) and further CSR isotype switchings were performed; and for Allele 2 (IGHD), homologous recombination was performed and initial allelic exclusion determined.

Gene esebssiwaago $T_{\mathrm{Q}}$-based simulation of 2-step and 1-step IGHD_-_ and IGHJ_ recombinations for allele 1 (IGHM) and allele 2 (IGHD)

For both alleles, 2-step (1, 2a, 2b) and 1-step D_-_ $\leftrightarrow$ J_ recombination simulations were performed.

For the 2-step $\mathrm{D}_{-}$-_ $^{\mathrm{S}} \mathrm{J} \mathrm{J}_{-}$stimulation: first, the nonfunctional $(n f)$ germline genes were determined (Step 1 of 2 ) and the IGHJ_ gene was determined (Step 1 of 2) to yield the recombined gene, IGHD_-_ (nf)-IGHJ_; second, the esebssiwaago $T_{\mathrm{Q}} \mathrm{S}$ for the remaining $I G H D_{-}-$and $I G H J_{-}$ genes with respect to IGHD_-_ (nf)-IGHI_ were determined; and third, these genes with respect to IGHD_- $(n f)-I G H J_{-}$, the remaining $I G H D_{-}-$with respect to IGHD_- $(n f)-I G H I_{-}$ (Step $2 \mathrm{a}$ of 2 ) and $I G H I_{-}$genes with respect to IGHD_(nf)-IGHI_ (Step 2a of 2) were recombined to yield the final step recombined gene, IGHD_- $-I G H I_{-}$(Step 2b of 2), ready for $I G H V_{-}{ }_{-} \leftrightarrow I G H D_{-}-I_{-} I G H J_{-}$.

For the 1-step $\mathrm{D}_{-}-_{-} \leftrightarrow \mathrm{J}_{-}$stimulation, the germline $I G H D_{-}$- $_{-}$and $I G H J_{-}$genes were recombined to yield the final recombined gene, IGHD_- -IGHJ_ (Step 1of 1), ready for $I G H V_{-}{ }_{-} \leftrightarrow I G H D_{-}-_{-} I G H J_{-}$.

Gene esebssiwaago $T_{\mathrm{Q}}$-based simulation of $I G H V_{-}-$and IGHD_-_-IGHJ_ recombinations for allele 1 (IGHM) and allele 2 (IGHD)

The most common variable genes were sampled. These genes included IGHV1-3, IGHV3-23, IGHV4-28, IGHV3-48, IGHV4-59 and IGHV4-61 with the exception of IGHV5-51 [18]. The IGHV_- to $I G H D_{-}{ }_{-}-I G H J_{-}$recombination events through further CSR isotype switching recombinations were performed for $V 1-3, V 3-23$, and V5-51.

Gene esebssiwaago $T_{\mathrm{Q}}$-based simulation of internal consensus recognition sequence (iCSR) recombination for allele 1 (IGHM)

For Allele 1 (IGHM) [11], internal consensus sequence recognition (iCSR) recombination was performed between MIR4537, MIR4507/MIR4538 and MIR4539 with 
respect to IGHV_-_-IGHD_-_-(IGHJ5)-IGHJ6. The complete recombined $\mathrm{V}(\mathrm{D}) \mathrm{J}$ gene is VDJ6 irrespective of whether it is the 1-step recombined IGHV_-_ - D_-_-J5 gene or the 2-step recombined $I G H V_{--}-D_{-}-$-J5-J6 gene.

Gene esebssiwaago $T_{\mathrm{Q}}$-based simulation of homologous recombination for allele 2 (IGHD) and determination of initial allelic exclusion

For Allele 2 (IGHD) [14], homologous recombination (HR)

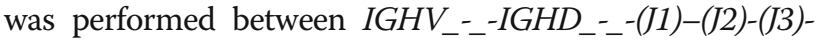
J4-(J5)-J6 (VDJ6) and IGHD with respect to IGHV--IGHD__-(J1)-(J2)-(J3)-J4-(J5)-J6. If there was no esebssiwaago $_{\mathrm{Q}}$ match (gene esebssiwaago $T_{\mathrm{Q}} \pm 0.015$ units) for homologous recombination, then further CSR was performed (as in Allele 1).

\section{Gene esebssiwaago $T_{\mathrm{Q}}$-based simulation of consensus recognition sequence (CSR) antibody isotype switchings for allele 1 (IGHM)}

For Allele 1 (IGHM) isotype switching [12, 13], consensus sequence recognition (CSR) recombination was performed if there was an esebssiwaago $T_{\mathrm{Q}}$ match between a downstream Ig heavy chain gene IGHG3, IGHG1, IGHA1, IGHG4/IGHG2, IGHE and IGHA2 with respect to VDJ6-IGHM ( \pm 0.015 units) and VDJ6-IGHM ( \pm 0.015 units). If there was an esebssiwaago $T_{\mathrm{Q}}$ match with a downstream gene for example with IGHG3, then the esebssiwaago $T_{\mathrm{Q}} \mathrm{s}$ for each remaining downstream Ig heavy chain gene IGHG1, IGHA1, IGHG4/IGHG2, IGHE and IGHA2 with respect to VDJ6-IGHG3 were determined, after which further esebssiwaago $T_{\mathrm{Q}}$ matches were determined and remaining downstream gene recombinations simulated.

\section{Results}

Germline IGH_genes

IGHA2 is a 3 episode, 7 initial SEB and final SEB gene that begins with a mesotropic SEB. IGHA2 has one instance of non-contributory anisotropy. IGHA2 is a $3 \mathrm{M}(7) \mathrm{NCA}$ gene with a final esebssiwaago $T_{\mathrm{Q}}$ of $0.10(0.099)$.

IGHE is a 3 episode, 7 initial SEB and final SEB gene that begins with an anisotropic SEB. IGHE is a 3 A (7) gene with a final esebssiwaago $T_{\mathrm{Q}}$ of $0.11(0.111)$.

IGHG4/IGHG2 is a 2 episode, 5 initial SEB and final SEB gene that begins with a mesotropic SEB. IGHG4/ IGHG2 has one instance of non-contributory anisotropy. IGHG4/IGHG2 is a $2 \mathrm{M}$ (5) NCA gene with a final esebssiwaago $_{\mathrm{Q}}$ of $0.16(0.163)$.

IGHA1 is a 3 episode, 7 initial SEB and final SEB gene that begins with a mesotropic SEB. IGHA1 is a $3 \mathrm{M}$ (7) gene with a final esebssiwaago $T_{\mathrm{Q}}$ of $0.13(0.131)$.
IGHG1 is a 3 episode, 7 initial SEB and final SEB gene that begins with an anisotropic SEB. IGHG1 is a 3 A (7) gene with a final esebssiwaago $T_{\mathrm{Q}}$ of $0.12(0.120)$.

IGHG3 is a 2 episode, 5 initial SEB and final SEB gene that begins with a anisotropic SEB. IGHG3 is a 2 A (5) gene with a final esebssiwaago $T_{\mathrm{Q}}$ of $0.08(0.075)$.

IGHD is a 3 episode, 7 initial SEB and final SEB gene that begins with a mesotropic SEB. IGHD is a $3 \mathrm{M}$ (7) gene with a final esebssiwaago $T_{\mathrm{Q}}$ of 0.09 (0.092).

$\boldsymbol{I G H} \boldsymbol{M}$ is a 3 episode, 7 initial SEB and final SEB gene that begins with anisotropic SEB. IGHM is a 3 A (7) gene with a final esebssiwaago $T_{\mathrm{Q}}$ of 0.09 (0.088).

MIR4539 is a 3 episode, 7 initial SEB and final SEB gene that begins with a mesotropic SEB. MIR4539 has one instance of non-contributory anisotropy. MIR4539 is a $3 \mathrm{M}(7) \mathrm{NCA}$ gene with a final esebssiwaago $T_{\mathrm{Q}}$ of 0.08 (0.076).

MIR4507/MIR4538 is a 3 episode, 7 initial SEB and final SEB gene that begins with an anisotropic SEB. MIR4507/MIR4538 has one instance of non-contributory anisotropy. MIR4507/MIR4538 is a 3 A (7) NCA gene with a final esebssiwaago $T_{\mathrm{Q}}$ of $0.08(0.081)$.

MIR4537 is a 3 episode, 7 initial SEB and final SEB gene that begins with a mesotropic SEB. MIR4537 has one instance of non-contributory anisotropy. MIR4537 is a $3 \mathrm{M}(7) \mathrm{NCA}$ gene with a final esebssiwaago $T_{\mathrm{Q}}$ of 0.06 (0.064) (Table 1, Additional file 2: Table S2).

\section{Germline IGHJ genes}

IGHJ6 is a 3 episode, 7 initial SEB and final SEB gene that begins with an anisotropic SEB. IGHJ6 is a 3 A (7) gene with a final esebssiwaago $T_{\mathrm{Q}}$ of 0.10 (0.097).

IGHJ5 is a 3 episode, 7 initial SEB and final SEB gene that begins with an anisotropic SEB. IGHJ5 is a 3 A (7) gene with a final esebssiwaago $T_{\mathrm{Q}}$ of 0.24 (0.235).

IGHJ4 is a 3 episode, 7 initial SEB and final SEB gene that begins with a mesotropic SEB. IGHJ4 has one instance of non-contributory anisotropy. IGHJ4 is a $3 \mathrm{M}$ (7) NCA gene with a final esebssiwaago $T_{\mathrm{Q}}$ of 0.11 (0.110).

IGHJ3 is a 3 episode, 7 initial SEB and final SEB gene that begins with a mesotropic SEB. IGHJ3 is a $3 \mathrm{M}$ (7) gene with a final esebssiwaago $T_{\mathrm{Q}}$ of 0.11 (0.112).

IGHJ2 is a 3 episode, 7 initial SEB and final SEB gene that begins with a mesotropic SEB. IGHJ2 is a $3 \mathrm{M}$ (7) gene with a final esebssiwaago $T_{\mathrm{Q}}$ of 0.11 (0.114).

IGHJ1 is a 3 episode, 7 initial SEB and final SEB gene that begins with a mesotropic SEB. IGHJ1 has one instance of non-contributory anisotropy. IGHJ1 is a $3 \mathrm{M}$ (7) NCA gene with a final esebssiwaago $T_{\mathrm{Q}}$ of 0.12 (0.116) (Table 2, Additional file 3: Table S3). 


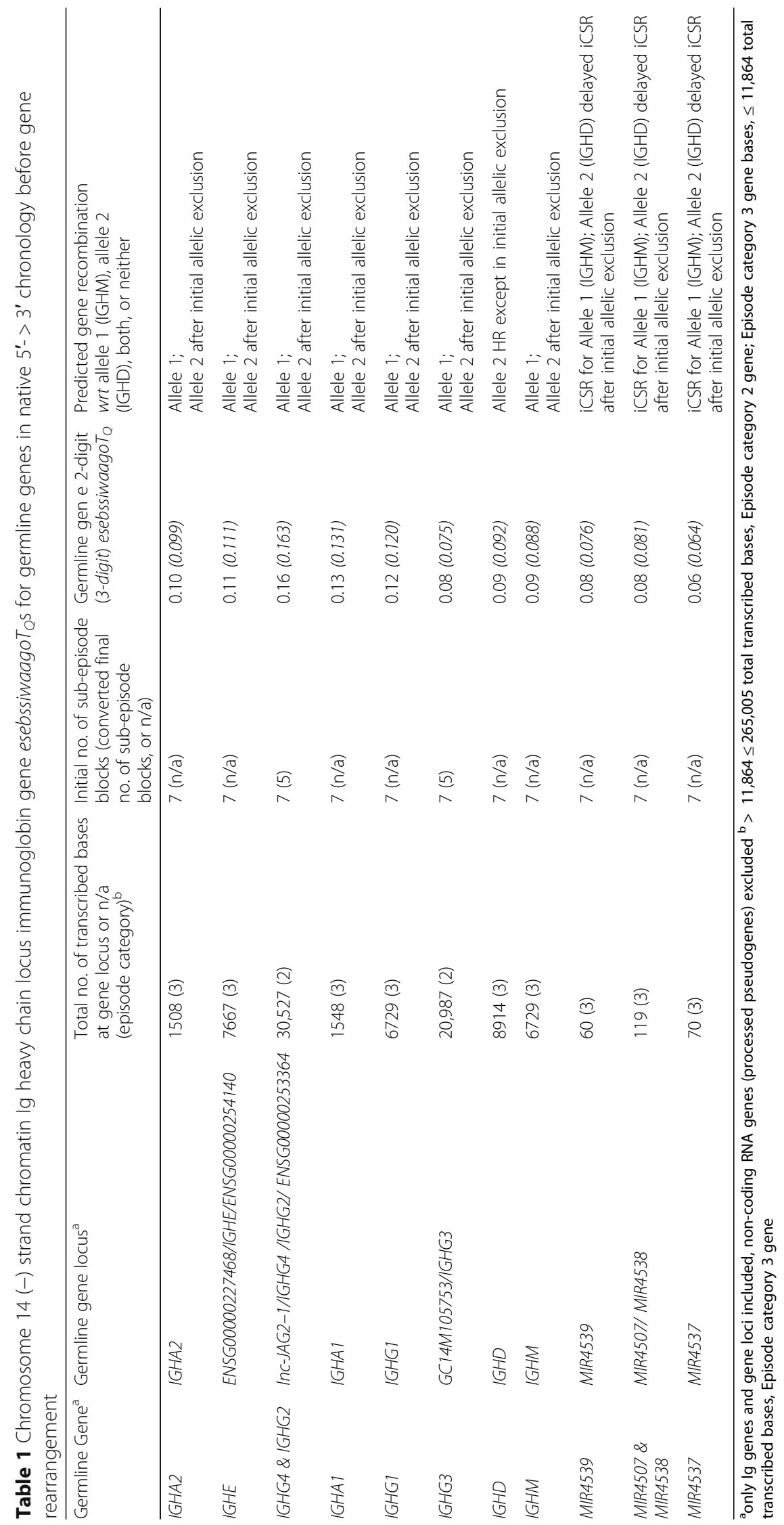


Germline non-functional IGHD1-20 (nf) gene for allele 1 (IGHM) 2-step (1 of 2 ) recombination

IGHD1-20 (nf) is a 3 episode, 7 initial SEB and $7^{*}$ final SEB gene that begins with an anisotropic SEB. IGHD1$20(n f)$ has one instance of non-contributory anisotropy at the ending. IGHD1-20 (nf) is a $3 \mathrm{~A}\left[7(+2): 7^{*}\right] \mathrm{NCA}^{*}$ gene with a final esebssiwaago $T_{\mathrm{Q}}$ of $0.41(0.406)$ (Table 3, Additional file 4: Table S4; Table 4).

\section{Germline functional IGHD_-_ genes un-involved in the} 1 st step of 2-step allele 1 (IGHM) recombination IGHD6-19 is a 3 episode, 7 initial and 5 final SEB gene that begins with a mesotropic SEB. IGHD6-19 has one instance of anisotropy converted-to-mesotropy, and one instance of indirect stIsotropy for mesotropy. IGHD6-19 is a $3 \mathrm{M}$ [7(-2): 5] ACM stIMfA gene with a final esebssiwaago $T_{\mathrm{Q}}$ of $0.31(0.309)$.

IGHD4-17 is a 3 episode, 7 initial and 9 final SEB gene that begins with an anisotropic SEB. IGHD4-17 has one instance of anisotropy converted-to-mesotropy. IGHD4-17 is a 3 A [7 (+2): 9] ACM with a final esebssiwaago $_{\mathrm{Q}}$ of $0.31(0.310)$.

IGHD3-16 is a 3 episode, 7 initial and final SEB gene that begins with a mesotropic SEB. IGHD3-16 has one instance of non-contributory anisotropy. IGHD3-16 is a $3 \mathrm{M}(7) \mathrm{NCA}$ gene with a final esebssiwaago $T_{\mathrm{Q}}$ of 0.31 (0.308).

IGHD3-10 is a 3 episode, 7 initial and final SEB gene that begins with an anisotropic SEB. IGHD3-10 has one instance of anisotropy converted-to-mesotropy, and non-contributory reverse/stIsotropy. IGHD3-10 is a $3 \mathrm{~A}$ (7) ACM NCstI gene with a final esebssiwaago $T_{\mathrm{Q}}$ of 0.34 (0.342).

IGHD2-8 is a 3 episode, 7 initial and final SEB gene that begins with a mesotropic SEB. IGHD2-8 has one instance of non-contributory reverse/ stIsotropy. IGHD2-8 is a $3 \mathrm{M}(7) \mathrm{NCstI}$ gene with a final esebssiwaago $_{\mathrm{Q}}$ of 0.30 . (0.295).
IGHD2-2 is a 3 episode, 7 initial and final SEB gene that begins with a mesotropic SEB. IGHD2-2 has one instance of non-contributory reverse/ stIsotropy. IGHD2-2 is a $3 \mathrm{M}$ (7) NCstI gene with a final esebssiwaago $_{\mathrm{Q}}$ of $0.30(0.301)$ (Table 3 , Additional file 4: Table S4; Table 4).

Germline non-functional IGHD4-11 (nf) gene and IGHD5-8 (nf) for allele 2 (IGHD) 2-step (1 of 2) recombination

IGHD4-11(nf) is a 3 episode, 7 initial SEB and final SEB gene that begins with an anisotropic SEB. IGHD4$11(n f)$ has one instance of anisotropy converted-tomesotropy IGHD4-11(nf) is a 3 A (7) ACM gene with a final esebssiwaago $T_{\mathrm{Q}}$ of 0.29 (0.293).

IGHD5-18 (nf) is a 3 episode, 7 initial and 6 final SEB gene that begins with a mesotropic SEB. IGHD5-18 (nf) has one instance of a noncontributory anisotropy, and one instance of indirect stIsotropy for anisotropy. IGHD5-18 (nf) is a $3 \mathrm{M}[7$ (-3): 4] NCA stIMfA gene with a final esebssiwaa$g o T_{\mathrm{Q}} 0.25$ (0.254) (Table 3, Additional file 4: Table S4; Table 5).

\section{Germline functional IGHD_-_ genes un-involved in the} 1st step of 2-step allele 2 (IGHD) recombination

IGHD3-9 is a 3 episode, 7 initial and 3 final SEB gene that begins with a mesotropic SEB. IGHD3-9 has one instance of anisotropy converted-to-mesotropy, one instance of non-contributory anisotropy, and one instance of non-contributory reverse/stIsotropy. IGHD3-9 is a $3 \mathrm{M}[7(-4): 3]$ ACM NCA NCstI gene with a final esebssiwaago $T_{\mathrm{Q}}$ of $0.24(0.239)$.

IGHD1-7 is a 3 episode, 7 initial and 5 final SEB gene that begins with an anisotropic SEB. IGHD1-7 has one instance of anisotropy converted-to-mesotropy, one instance of non-contributory anisotropy, and non-contributory reverse/stIsotropy. IGHD1-7 is a 3 A [7 (-2): 5] ACM NCA $\mathrm{NCstI}$ gene with a final esebssiwaago $T_{\mathrm{Q}}$ of $0.28(0.276)$.

Table 2 Chromosome $14(-)$ strand chromatin Ig heavy chain locus joining gene esebssiwaagoTos for germline genes in native 5'- > 3' chronology before gene rearrangement

\begin{tabular}{|c|c|c|c|c|c|}
\hline $\begin{array}{l}\text { Germline } \\
\text { Gene }^{a}\end{array}$ & $\begin{array}{l}\text { Germline gene } \\
\text { locus }^{\mathrm{a}}\end{array}$ & $\begin{array}{l}\text { Total no. of transcribed } \\
\text { bases at gene locus or } \\
\text { n/a (episode category) }\end{array}$ & $\begin{array}{l}\text { Initial no. of sub-episode } \\
\text { blocks (converted final } \\
\text { no. of sub-episode blocks, or n/a) }\end{array}$ & $\begin{array}{l}\text { Germline gen e } \\
\text { 2-digit (3-digit) } \\
\text { esebssiwaagotQ }\end{array}$ & $\begin{array}{l}\text { Predicted gene recombination } \\
\text { wrt allele } 1 \text { (IGHM), allele } 2 \\
\text { (IGHD), both, or neither }\end{array}$ \\
\hline IGHJ6 & IGHJ6 & $65(3)$ & $7(\mathrm{n} / \mathrm{a})$ & $0.10(0.097)$ & Allele 1 ( $\mathrm{D} \rightarrow$ J step 2 of 2 for IGHM) \\
\hline IGHJ5 & IGHJ5 & $53(3)$ & $7(n / a)$ & $0.24(0.235)$ & $\begin{array}{l}\text { Allele } 1(D \rightarrow \text { J step } 1 \text { of } 1 \& \text { step } 1 \text { of } \\
2 \text { for IGHM) }\end{array}$ \\
\hline IGHJ4 & IGHJ4 & $50(3)$ & $7(n / a)$ & $0.11(0.110)$ & Allele 2 ( $\mathrm{D} \rightarrow$ J step 2 of 2 for IGHD) \\
\hline IGHJ3 & IGHJ3 & $52(3)$ & $7(n / a)$ & $0.11(0.112)$ & Allele 2 ( $\mathrm{D} \rightarrow$ J step 1 of 1 for IGHD) \\
\hline IGHJ2 & $\mid G H J 2$ & $55(3)$ & $7(\mathrm{n} / \mathrm{a})$ & $0.11(0.114)$ & $\begin{array}{l}\text { Allele } 2(D \rightarrow \text { J step } 1 \text { of } 1 \& \text { step } 1 \text { of } \\
2 \text { for IGHD) }\end{array}$ \\
\hline |GHJI & $\mid G H J 1$ & $54(3)$ & $7(n / a)$ & $0.12(0.116)$ & Allele 2 ( $\rightarrow$ J step 1 of 1 for IGHD) \\
\hline
\end{tabular}

${ }^{a}$ only lg genes and gene loci included, non-coding RNA genes (processed pseudogenes) excluded ${ }^{\mathrm{b}}>11,864 \leq 265,005$ total transcribed bases, Episode category 2 gene; Episode category 3 gene bases, $\leq 11,864$ total transcribed bases, Episode category 3 gene 
Table 3 Chromosome 14 (-) strand chromatin Ig heavy chain locus diversity gene esebssiwaagot $S$ for germline genes in native $5^{\prime}->$ 3' chronology before gene rearrangement

\begin{tabular}{|c|c|c|c|c|c|}
\hline Germline Gene 1 nf & Germline gene locus $^{a}$ & $\begin{array}{l}\text { Total no. of transcribed } \\
\text { bases at gene locus or } \\
\text { n/a (episode category) }\end{array}$ & $\begin{array}{l}\text { Initial no. of sub-episode } \\
\text { blocks (converted final no. of } \\
\text { sub-episode blocks, or n/a) }\end{array}$ & $\begin{array}{l}\text { Germline gene } \\
\text { 2-digit (3-digit) } \\
\text { esebssiwaagoT } T_{Q}^{c}\end{array}$ & $\begin{array}{l}\text { Predicted gene recombination } \\
\text { wrt allele } 1(\mathrm{IGHM}) \text {, allele } 2 \\
\text { (IGHD), both, or neither }\end{array}$ \\
\hline IGHD7-27 & IGHD7-27 & $11(3)$ & $7(n / a)$ & $0.17(0.165)^{c}$ & Neither allele 1 nor allele 2 \\
\hline IGHD1-26 & IGHD1-26 & $20(3)$ & 7 (n/a) & $0.22(0.217)$ & $\begin{array}{l}\text { Allele } 2(D \rightarrow \text { J step } 1 \text { of } 1 \text { for } \\
\text { IGHD) }\end{array}$ \\
\hline IGHD6-25 & IGHD6-25 & $18(3)$ & $7(n / a)$ & $0.17(0.172)$ & $\begin{array}{l}\text { Allele } 2(\mathrm{D} \rightarrow \mathrm{J} \text { step } 1 \text { of } 1 \text { for } \\
\text { IGHD) }\end{array}$ \\
\hline $\begin{array}{l}\text { IGHD5-24, IGHD4- } \\
23 \& \text { IGHD3-22 }\end{array}$ & $\begin{array}{l}\text { Inc-BRF1-1 \{IGHD5-24; } \\
\text { IGHD4-23; IGHD3-22) }\end{array}$ & $2856(3)$ & $7(5)$ & $0.22(0.216)$ & $\begin{array}{l}\text { Allele } 2 \text { ( } D \rightarrow \text { J step } 1 \text { of } 1 \text { for } \\
\text { IGHD) }\end{array}$ \\
\hline IGHD2-21 & IGHD2-21 & $28(3)$ & $7(n / a)$ & $0.20(0.205)$ & $\begin{array}{l}\text { Allele } 2 \text { ( } D \rightarrow \text { J step } 1 \text { of } 1 \text { for } \\
\text { IGHD) }\end{array}$ \\
\hline $1 G H D 1-20^{\text {nf }}$ & IGHD1-20 & $17(3)$ & $7(7)$ & $0.41(0.406)$ & $\begin{array}{l}\text { Allele } 1 \text { non-functional (D } \rightarrow \text { J } \\
\text { step } 1 \text { of } 2 \text { for IGHM) }\end{array}$ \\
\hline IGHD6-19 & IGHD6-19 & $21(3)$ & $7(5)$ & $0.31(0.309)$ & $\begin{array}{l}\text { Allele } 1(\mathrm{D} \rightarrow \text { J step } 2 \text { of } 2 \text { for } \\
\text { IGHM wrt IGHJ5-IGHD1-20) }\end{array}$ \\
\hline IGHD5-18 & IGHD5-18 & $20(3)$ & $7(4)$ & $0.25(0.254)$ & $\begin{array}{l}\text { Allele } 2 \text { non-functional }(D \rightarrow J \\
\text { step } 1 \text { of } 2 \text { for } I G H D)\end{array}$ \\
\hline IGHD4-17 & IGHD4-17 & $16(3)$ & $7(9)$ & $0.31(0.310)$ & $\begin{array}{l}\text { Allele } 1(\mathrm{D} \rightarrow \text { J step } 2 \text { of } 2 \text { for } \\
\text { IGHM wrt IGHJ5-IGHD1-20) }\end{array}$ \\
\hline IGHD3-16 & IGHD3-16 & $37(3)$ & 7 (n/a) & $0.31(0.308)$ & $\begin{array}{l}\text { Allele } 1(\mathrm{D} \rightarrow \mathrm{J} \text { step } 2 \text { of } 2 \text { for } \\
\text { IGHM wrt IGHJ5-IGHD1-20) }\end{array}$ \\
\hline IGHD2-15 & IGHD2-15 & $31(3)$ & $7(\mathrm{n} / \mathrm{a})$ & $0.29(0.294)$ & $\begin{array}{l}\text { Allele } 1(\mathrm{D} \rightarrow \mathrm{J} \text { step } 1 \text { of } 1 \text { for } \\
\text { IGHM }\end{array}$ \\
\hline |GHD1-14 & IGHD1-14 & $17(3)$ & 7 (n/a) & $0.29(0.292)$ & $\begin{array}{l}\text { Allele } 1(\mathrm{D} \rightarrow \mathrm{J} \text { step } 1 \text { of } 1 \text { for } \\
\text { IGHM }\end{array}$ \\
\hline IGHD6-13 & IGHD6-13 & $21(3)$ & 7 (n/a) & $0.29(0.286)$ & $\begin{array}{l}\text { Allele } 2 \text { ( } D \rightarrow \text { J step } 1 \text { of } 1 \text { for } \\
\text { IGHM }\end{array}$ \\
\hline IGHD5-12 & IGHD5-12 & $23(3)$ & $7(n / a)$ & $0.22(0.218)$ & $\begin{array}{l}\text { Allele } 2 \text { (D } \rightarrow \text { J step } 1 \text { of } 1 \text { for } \\
\text { IGHD) }\end{array}$ \\
\hline $\mid G H D 4-11^{n f}$ & |GHD4-11 & $16(3)$ & 7 (n/a) & $0.29(0.293)$ & $\begin{array}{l}\text { Allele } 2 \text { non-functional }(D \rightarrow J \\
\text { step } 1 \text { of } 2 \text { for IGHD) }\end{array}$ \\
\hline IGHD3-10 & IGHD3-10 & $31(3)$ & 7 (n/a) & $0.34(0.342)$ & $\begin{array}{l}\text { Allele } 1(\mathrm{D} \rightarrow \mathrm{J} \text { step } 2 \text { of } 2 \text { for } \\
\text { IGHM wrt IGHJ5-IGHD1-20) }\end{array}$ \\
\hline IGHD3-9 & IGHD3-9 & $31(3)$ & $7(3)$ & $0.24(0.239)$ & $\begin{array}{l}\text { Allele } 2(\mathrm{D} \rightarrow \mathrm{J} \text { step } 2 \text { of } 2 \text { for } \\
\text { IGHD wrt IGHJ2-IGHD4-11) }\end{array}$ \\
\hline IGHD2-8 & IGHD2-8 & $31(3)$ & 7 (n/a) & $0.30(0.295)$ & $\begin{array}{l}\text { Allele } 1(\mathrm{D} \rightarrow \text { J step } 2 \text { of } 2 \text { for } \\
\text { IGHM wrt IGHJ5-IGHD1-20) }\end{array}$ \\
\hline IGHD1-7 & |GHD1-7 & $17(3)$ & $7(5)$ & $0.28(0.276)$ & $\begin{array}{l}\text { Allele } 2(\mathrm{D} \rightarrow \mathrm{J} \text { step } 2 \text { of } 2 \text { for } \\
\text { IGHD wrt IGHJ2-IGHD4-11) }\end{array}$ \\
\hline IGHD6-6 & IGHD6-6 & $18(3)$ & 7 (n/a) & $0.27(0.275)$ & $\begin{array}{l}\text { Allele } 2(\mathrm{D} \rightarrow \mathrm{J} \text { step } 2 \text { of } 2 \text { for } \\
\text { IGHD wrt IGHJ2-IGHD4-11) }\end{array}$ \\
\hline IGHD5-5 & IGHD5-5 & $20(3)$ & 7 (n/a) & $0.23(0.233)$ & $\begin{array}{l}\text { Allele } 2(\mathrm{D} \rightarrow \mathrm{J} \text { step } 2 \text { of } 2 \text { for } \\
\text { IGHD wrt IGHJ2-IGHD5-18) }\end{array}$ \\
\hline IGHD4-4 & IGHD4-4 & $16(3)$ & 7 (n/a) & $0.26(0.258)$ & $\begin{array}{l}\text { Allele } 2(\mathrm{D} \rightarrow \mathrm{J} \text { step } 2 \text { of } 2 \text { for } \\
\text { IGHD wrt IGHJ2-IGHD4-11) }\end{array}$ \\
\hline IGHD3-3 & IGHD3-3 & $31(3)$ & $7(9)$ & $0.24(0.243)$ & $\begin{array}{l}\text { Allele } 1(\mathrm{D} \rightarrow \mathrm{J} \text { step } 2 \text { of } 2 \text { for } \\
\text { IGHD wrt IGHJ2-IGHD4-11) }\end{array}$ \\
\hline IGHD2-2 & IGHD2-2 & $31(3)$ & $7(n / a)$ & $0.30(0.301)$ & $\begin{array}{l}\text { Allele } 1 \text { (D } \rightarrow \text { J step } 2 \text { of } 2 \text { for } \\
\text { IGHM wrt IGHJ5-IGHD1-20) }\end{array}$ \\
\hline IGHD1-1 & |GHDI-1 & $17(3)$ & $7(7)$ & $0.23(0.233)$ & $\begin{array}{l}\text { Allele } 2(\mathrm{D} \rightarrow \mathrm{J} \text { step } 2 \text { of } 2 \text { for } \\
\text { IGHD wrt IGHJ2-IGHD5-18) }\end{array}$ \\
\hline
\end{tabular}




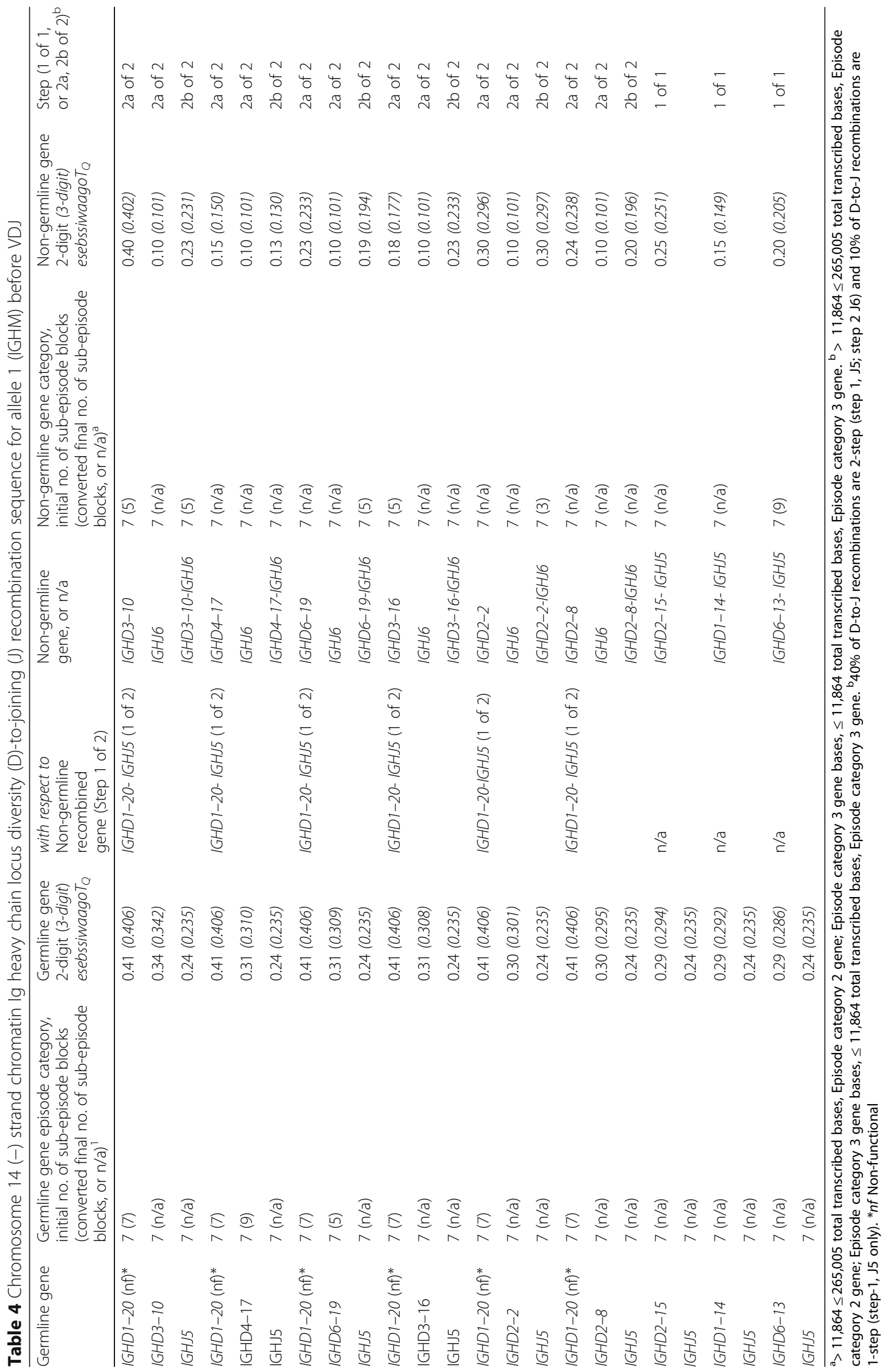




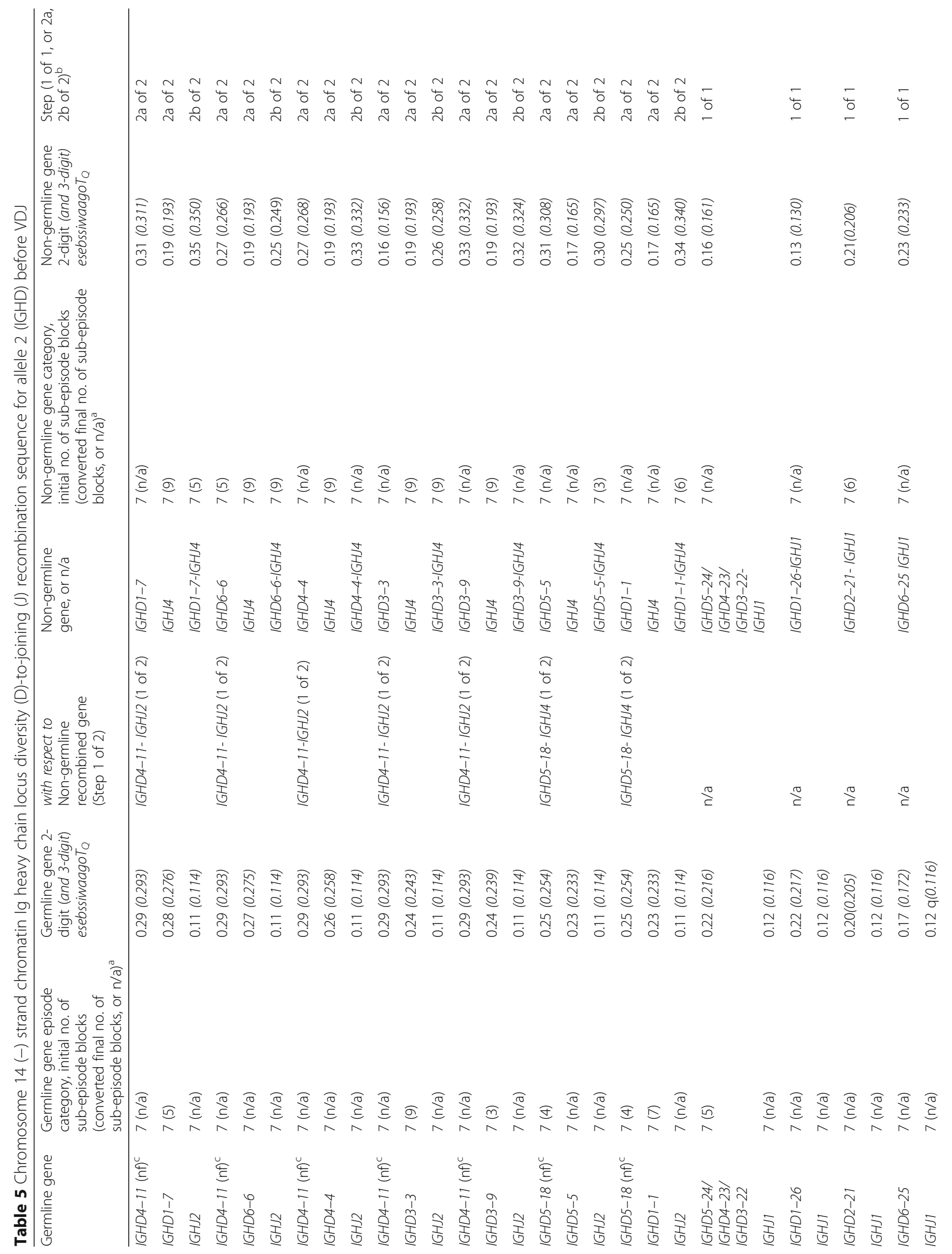




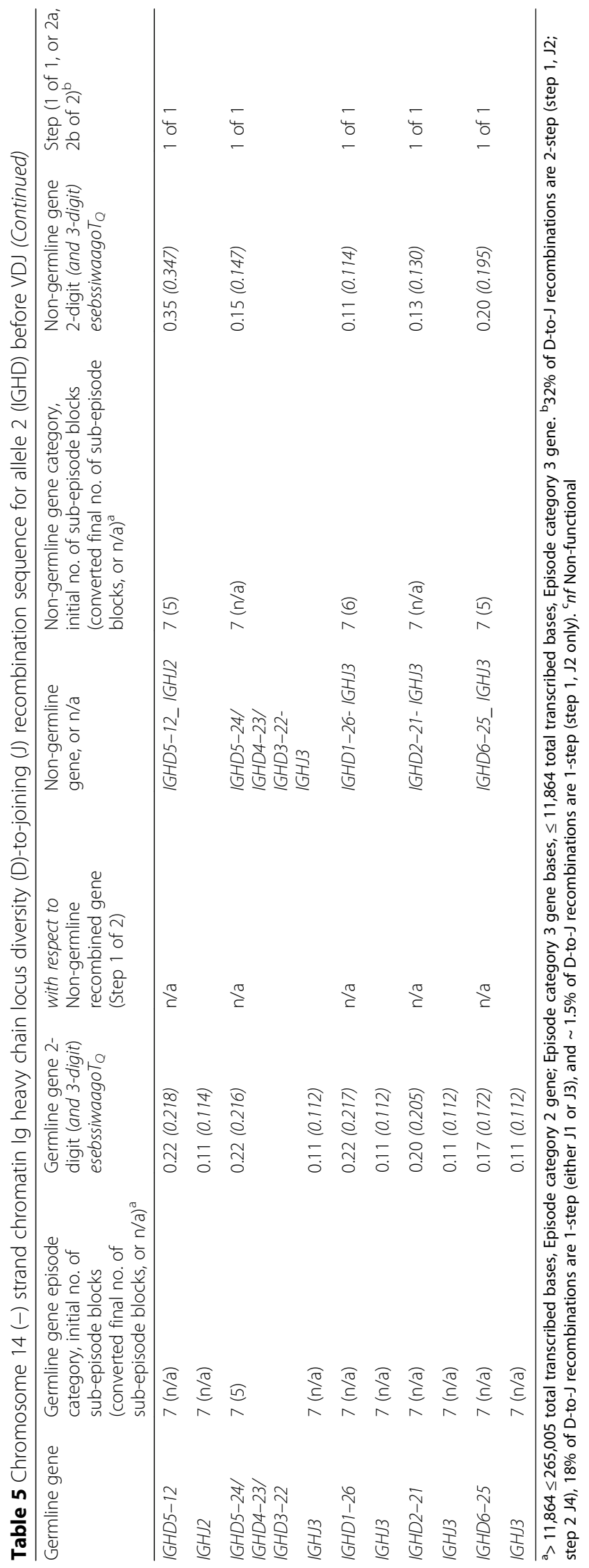


IGHD6-6 is a 3 episode, 7 initial and final SEB gene that begins with a mesotropic SEB. IGHD6-6 has one instance of indirect stIsotropy for mesotropy. IGHD6-19 is a $3 \mathrm{M}$ (7) stIMfM gene with a final esebssiwaago $T_{\mathrm{Q}}$ of $0.27(0.275)$.

IGHD5-5 is a 3 episode, 7 initial and final SEB gene that begins with a mesotropic SEB. IGHD5-5 has one instance of non-contributory reverse/stIsotropy. IGHD5-5 is a $3 \mathrm{M}(7) \mathrm{NCstI}$ gene with a final esebssiwaago $T_{\mathrm{Q}}$ of 0.23 (0.233).

IGHD4-4 is a 3 episode, 7 initial and final SEB gene that begins with a mesotropic SEB. IGHD4-4 has two instances of non-contributory reverse/stIsotropy. IGHD4-4 is a $3 \mathrm{M}(7) \mathrm{NCstI} \times 2$ gene with a final esebssiwaago $T_{\mathrm{Q}}$ of 0.26 (0.258).

IGHD3-3 is a 3 episode, 7 initial and 9 final SEB gene that begins with a mesotropic SEB. IGHD3-3 has one instance of non-contributory anisotropy, and one instance of non-contributory reverse/stIsotropy. IGHD3-3 is a $3 \mathrm{M}[7(+2): 9] \mathrm{NCA}$ NCstI gene with a final esebssiwaago $_{\mathrm{Q}}$ of $0.24(0.243)$.

IGHD1-1 is a 3 episode, 7 initial SEB and $7^{*}$ final SEB gene that begins with a mesotropic SEB. IGHD1-1 has one instance of anisotropy converted-to-mesotropy preceding ending confirmation (anisotropic SEB no. 8), and three instances of non-contributory reverse/stIsotropy. IGHD1-1 is a $3 \mathrm{M}\left[7(+1): 7^{*}\right] \mathrm{ACM}^{*} \mathrm{NCstI}$ gene with a final esebssiwaago $T_{\mathrm{Q}}$ of $0.23(0.233)$ (Table 3, Additional file 4: Table S4; Table 5).

\section{Germline non-functional IGHD_-_ gene}

IGHD7 $-27^{+}$is a 3 episode, 7 initial SEB and final SEB gene that begins with a mesotropic SEB. IGHD7-27 $7^{\dagger}$ is a $3 \mathrm{M}(7)$ gene with a final esebssiwaago $T_{\mathrm{Q}}$ of. $0.17(0.165)^{\dagger}$ (Table 3, Additional file 4: Table S4).

Germline IGHJ5 gene for allele 1 (IGHM) 2-step (1 of 2) recombination [and for allele 1 (IGHM) 1-step recombination] IGHJ5 is a $3 \mathrm{~A}(7)$ gene with a final esebssiwaago $T_{\mathrm{Q}}$ of 0.24 (0.235) (Table 2, Additional file 3: Table S3; Table 4).

\section{Germline non-functional IGHD1-20 (nf) gene for allele 1} (IGHM) 2-step (1 of 2 ) recombination

IGHD1-20 (nf) is a 3 A [7 (+2): 7*] NCA* gene with a final esebssiwaago $T_{\mathrm{Q}}$ of 0.41 (0.406) (Table 3, Additional file 4: Table S4; Table 4).

IGHJ6 gene with respect to IGHD1-20 (nf)-IGHJ5 for allele 1 (IGHM) 2-step (2a of 2 ) recombination

IGHJ6 with respect to IGHD1-20-IGHJ5 is a 3 episode, 7 initial and final SEB gene that begins with an anisotropic SEB. IGHJ6 with respect to IGHD1-20-IGHJ5 is a 3 A (7) gene with a final esebssiwaago $T_{\mathrm{Q}}$ of $0.10(0.101)$ (Table 4, Additional file 5: Table S5).
IGHD_-_ genes with respect to IGHD1-20 (nf)-IGHJ5 for allele 1 (IGHM) 2-step ( $2 \mathrm{a}$ of 2 ) recombination IGHD3-10 with respect to IGHD1-20-IGHJ5 is a 3 episode, 7 initial and 5 final SEB gene that begins with an anisotropic SEB. IGHD3-10 with respect to IGHD120-IGHJ5 has one instance of non-contributory anisotropy, and one instance of non-contributory reverse/stIsotropy. IGHD3-10 with respect to IGHD1-20-IGHJ5 is a 3 A [7(-2): 5] NCA NCstI gene with a final esebssiwaago $T_{\mathrm{Q}}$ of $0.40(0.402)$.

IGHD4-17 with respect to IGHD1-20-IGHJ5 is a 3 episode, 7 initial and final SEB gene that begins with a mesotropic SEB. IGHD4-17 with respect to IGHD1-20IGHJ5 has one instance of non-contributory anisotropy. IGHD4-17 with respect to IGHD1-20-IGHJ5 is a $3 \mathrm{M}$ (7) $\mathrm{NCA}$ gene with a final esebssiwaago $T_{\mathrm{Q}}$ of 0.15 (0.150).

IGHD6-19 with respect to IGHD1-20-IGHJ5 is a 3 episode, 7 initial and 3 final SEB gene that begins with a mesotropic SEB. IGHD6-19 with respect to IGHD1-20IGHJ5 is a $3 \mathrm{M}(7)$ gene with a final esebssiwaago $T_{\mathrm{Q}}$ of 0.23 (0.233).

IGHD3-16 with respect to IGHDI-20-IGHJ5 is a 3 episode, 7 initial and 5 final SEB gene that begins with a mesotropic SEB. IGHD3-16 with respect to IGHD1-20IGHJ5 has one instance of anisotropy converted-tomesotropy. IGHD3-16 with respect to IGHD1-20-IGHJ5 is a $3 \mathrm{M}$ [7(-2): 5] ACM gene with a final esebssiwaa$g o T_{\mathrm{Q}}$ of $0.18(0.177)$.

IGHD2-2 with respect to IGHD1-20-IGHJ5 is a 3 episode, 7 initial and 3 final SEB gene that begins with a mesotropic SEB. IGHD2-2 with respect to IGHD1-20IGHJ5 has one instance of non-contributory reverse/stIsotropy. IGHD3-9 is a $3 \mathrm{M}$ (7) NCstI gene with a final esebssiwaago $T_{\mathrm{Q}}$ of 0.30 (0.296).

IGHD2-8 with respect to IGHD1-20-IGHJ5 is a 3 episode, 7 initial and 3 final SEB gene that begins with a mesotropic SEB. IGHD2-8 with respect to IGHD1-20IGHJ5 has one instance of non-contributory anisotropy, and one instance of non-contributory reverse/stIsotropy. IGHD2-8 with respect to IGHD1-20-IGHJ5 is a $3 \mathrm{M}$ (7) NCA NCstI gene with a final esebssiwaago $T_{\mathrm{Q}}$ of 0.24 (0.238) (Table 4, Additional file 5: Table S5).

IGHD_-_-IGHJ_genes for allele 1 (IGHM) 2-step (2b of 2) recombination

IGHD3-10-IGHJ6 is a 3 episode, 7 initial and 5 final SEB gene that begins with an anisotropic SEB. IGHD310-IGHJ6 has one instance of anisotropy converted-tomesotropy. IGHD3-10-IGHJ6 is a 3 A [7(-2): 5] ACM gene with a final esebssiwaago $T_{\mathrm{Q}}$ of $0.23(0.231)$.

IGHD4-17-IGHJ6 is a 3 episode, 7 initial and final SEB gene that begins with a mesotropic SEB. IGHD417-IGHJ6 has one instance of non-contributory 
anisotropy. IGHD4-17-IGHJ6 is a $3 \mathrm{M}$ (7) NCA gene with a final esebssiwaago $T_{\mathrm{Q}}$ of $0.13(0.130)$.

IGHD6-19-IGHJ6 is a 3 episode, 7 initial and 5 final SEB gene that begins with a mesotropic SEB. IGHD619-IGHJ6 has one instance of anisotropy converted-tomesotropy. IGHD6-19-IGHJ6 is a $3 \mathrm{M}$ [7(-2): 5] ACM gene with a final esebssiwaago $T_{\mathrm{Q}}$ of 0.19 (0.194).

IGHD3-16-IGHJ6 is a 3 episode, 7 initial SEB and final SEB gene that begins with a mesotropic SEB. IGHD3-16-IGHJ6 is a $3 \mathrm{M}$ (7) gene with a final esebssiwaago $_{\mathrm{Q}}$ of $0.23(0.233)$.

IGHD2-2-IGHJ6 is a 3 episode, 7 initial and 3 final SEB gene that begins with a mesotropic SEB. IGHD2-2IGHJ6 has one instance of non-contributory anisotropy, and one instance of indirect stIsotropy for anisotropy. IGHD2-2-IGHJ6 is a $3 \mathrm{M}$ [7(-4): 3] NCA stIMfA gene with a final esebssiwaago $T_{\mathrm{Q}}$ of $0.30(0.297)$.

IGHD2-8-IGHJ6 is a 3 episode, 7 initial SEB and final SEB gene that begins with a mesotropic SEB. IGHD2-8-IGHJ6 is a $3 \mathrm{M}$ (7) gene with a final esebssiwagoo $_{\mathrm{Q}}$ of $0.20(0.196)$ (Table 4, Additional file 5: Table S5).

Germline IGHJ2 gene for allele 2 (IGHD) 2-step (1 of 2) [and for allele 2 (IGHD) 1-step recombination]

IGHJ2 is a $3 \mathrm{M}$ (7) gene with a final esebssiwaago $T_{\mathrm{Q}}$ of 0.11 (0.114) (Table 2, Additional file 3: Table S3; Table 5).

Germline non-functional IGHD_-_ (nf) genes for allele 2 (IGHD) 2-step (1 of 2) recombination

IGHD4-11(nf) is a 3 A (7) ACM gene with a final esebssiwaagot $_{\mathrm{Q}}$ of 0.29 (0.293).

IGHD5-18 (nf) is a $3 \mathrm{M}$ [7 (-3): 4] NCA stIMfA gene with a final esebssiwaago $T_{\mathrm{Q}} 0.25$ (0.254) (Table 3, Additional file 4: Table S4; Table 5).

IGHJ4 gene with respect to IGHD4-11 (nf)-IGHJ2 and IGHJ4 gene with respect to IGHD5-18 (nf)-IGHJ2 for allele 2 (IGHD) 2-step (2a of 2) recombination

IGHJ4 with respect to IGHD4-11-IGHJ2 is a 3 episode, 7 initial and 9 final SEB gene that begins with an mesotropic SEB. IGHJ4 with respect to IGHD4-11-IGHJ2 has one instance of anisotropy converted-to-mesotropy. IGHJ4 with respect to IGHD4-11-IGHJ2 is a $3 \mathrm{M}[7(+2): 9] \mathrm{ACM}$ with a final esebssiwaago $T_{\mathrm{Q}} 0.19$ (0.193).

IGHJ4 with respect to IGHD5-18-IGHJ4 is a 3 episode, 7 initial SEB and final SEB gene that begins with a mesotropic SEB. IGHJ4 with respect to IGHD5-18-IGHJ4 is a $3 \mathrm{M}(7)$ gene with a final esebssiwaago $T_{\mathrm{Q}}$ of 0.17 (0.165) (Table 5, Additional file 6: Table S6).
IGHD_-- genes with respect to IGHD_-- (nf)-IGHJ2 for allele 2 (IGHD) 2-step (2a of 2) recombination

IGHD1-7 with respect to IGHD4-11-IGHJ2 is a 3 episode, 7 initial and final SEB gene that begins with an anisotropic SEB. IGHD1-7 with respect to IGHD4-11$I G H J 2$ has one instance of non-contributory reverse/stIsotropy. IGHD1-7 with respect to IGHD4-11-IGHJ2 is a 3 A (7) NCstI gene with a final esebssiwaago $T_{\mathrm{Q}}$ of 0.31 (0.311).

IGHD6-6 with respect to IGHD4-11-IGHJ2 is a 3 episode, 7 initial and 5 final SEB gene that begins with a mesotropic SEB. IGHD6-6 with respect to IGHD4-11IGHJ2 has one instance of anisotropy converted-tomesotropy, one instance of non-contributory anisotropy, and one instance of non-contributory reverse/stIsotropy. IGHD6-6 with respect to IGHD4-11-IGHJ2 is a $3 \mathrm{M}$ [7(-2): 5] ACM NCA NCstI gene with a final esebssiwaago $T_{\mathrm{Q}}$ of $0.27(0.266)$.

IGHD4-4 with respect to IGHD4-11-IGHJ2 is a 3 episode, 7 initial and final SEB gene that begins with a mesotropic SEB. IGHD4-4 with respect to IGHD4-11$I G H J 2$ has one instance of non-contributory reverse/stIsotropy. IGHD4-4 with respect to IGHD4-11-IGHJ2 is a $3 \mathrm{M}$ (7) NCstI gene with a final esebssiwaago $T_{\mathrm{Q}}$ of 0.27 (0.268).

IGHD3-3 with respect to IGHD4-11-IGHJ2 is a 3 episode, 7 initial and final SEB gene that begins with a mesotropic SEB. IGHD3-3 with respect to IGHD4-11$I G H J 2$ has one instance of non-contributory reverse/stIsotropy. IGHD3-3 with respect to IGHD4-11-IGHJ2 is a $3 \mathrm{M}(7) \mathrm{NCstI}$ gene with a final esebssiwaago $T_{\mathrm{Q}}$ of 0.16 (0.156).

IGHD3-9 with respect to IGHD4-11-IGHJ2 is a 3 episode, 7 initial and final SEB gene that begins with a mesotropic SEB. IGHD3-9 with respect to IGHD4-11IGHJ2 has one instance of anisotropy converted-tomesotropy. IGHD3-9 with respect to IGHD4-11-IGHJ2 a $3 \mathrm{M}(7) \mathrm{ACM}$ gene with a final esebssiwaago $T_{\mathrm{Q}}$ of 0.33 (0.332).

IGHD5-5 with respect to IGHD5-18-IGHJ2 is a 3 episode, 7 initial and final SEB gene that begins with a mesotropic SEB. IGHD5-5 with respect to IGHD5-18IGHJ2 has one instance of anisotropy converted-tomesotropy, and one instance of non-contributory reverse/ stIsotropy. IGHD5-5 with respect to IGHD5-18-IGHJ2 is a $3 \mathrm{M}$ (7) ACM NCstI gene with a final esebssiwaago $T_{\mathrm{Q}}$ of $0.31(0.308)$.

IGHD1-1 with respect to IGHD5-18-IGHJ2 is a 3 episode, 7 initial and final SEB gene that begins with a mesotropic SEB. IGHD1-1 with respect to IGHD518-IGHJ2 has one instance of non-contributory reverse/stIsotropy, and one instance of indirect stIsotropy for mesotropy. IGHD1-1 with respect to IGHD5-18-IGHJ2 is a $3 \mathrm{M}$ (7) NCstI stIMfM gene 
with a final esebssiwaago $T_{\mathrm{Q}}$ of $0.25(0.250)$ (Table 5, Additional file 6: Table S6).

IGHD_-_-IGHJ_genes for allele 2 (IGHD) 2-step (2b of 2) recombination

IGHD1-7-IGHJ4 is a 3 episode, 7 initial and 5 final SEB gene that begins with an anisotropic SEB. IGHD1-7-IGHJ4 has one instance of anisotropy converted-to-mesotropy, and one instance of indirect stIsotropy for mesotropy. IGHD1-7-IGHJ4 is a $3 \mathrm{~A}$ [7(-2): 5] ACM stIMfM gene with a final esebssiwaa$g o T_{\mathrm{Q}}$ of 0.35 (0.350).

IGHD6-6-IGHJ4 is a 3 episode, 7 initial and 9 final SEB gene that begins with a mesotropic SEB. IGHD6-6IGHJ4 has one instance of anisotropy converted-tomesotropy. IGHD6-6-IGHJ4 is a $3 \mathrm{M}$ [7(+2): 9] ACM gene with a final esebssiwaago $T_{\mathrm{Q}}$ of $0.25(0.249)$.

IGHD4-4-IGHJ4 is a 3 episode, 7 initial and final SEB gene that begins with anisotropic SEB. IGHD4-4-IGHJ4 has one instance of anisotropy converted-to-mesotropy. IGHD4-4-IGHJ4 is a 3 A (7) ACM gene with a final esebssiwaago $T_{\mathrm{Q}}$ of $0.33(0.332)$.

IGHD3-3-IGHJ4 is a 3 episode, 7 initial and 9 final SEB gene that begins with a mesotropic SEB. IGHD3-3IGHJ4 has one instance of anisotropy converted-tomesotropy. IGHD3-3-IGHJ4 is a $3 \mathrm{M}$ [7(+2): 9] ACM gene with a final esebssiwaago $T_{\mathrm{Q}}$ of $0.26(0.258)$.

IGHD3-9-IGHJ4 is a 3 episode, 7 initial and final SEB gene that begins with a mesotropic SEB. IGHD3-3IGHJ4 is a $3 \mathrm{M}(7)$ gene with a final esebssiwaago $T_{\mathrm{Q}}$ of 0.32 (0.324).

IGHD5-5-IGHJ4 is a 3 episode, 7 initial and 3 final SEB gene that begins with a mesotropic SEB. IGHD5-5IGHJ4 has one instance of a non-contributory anisotropy, and one instance of indirect stIsotropy for anisotropy. IGHD5-5-IGHJ4 is a $3 \mathrm{M}$ [7(-4): 3] NCA stIMfA gene with a final esebssiwaago $T_{\mathrm{Q}}$ of $0.30(0.297)$.

IGHD1-1-IGHJ4 is a 3 episode, 7 initial and 6 final SEB gene that begins with an anisotropic SEB. IGHD11-IGHJ4 has one instance of a non-contributory anisotropy. IGHD1-1-IGHJ4 is a 3 A [7(-1): 6] NCA gene with a final esebssiwaago $T_{\mathrm{Q}}$ of $0.34(0.340)$ (Table 5, Additional file 6: Table S6).

Germline IGHJ5 gene for allele 1 (IGHM) allele 1 (IGHM) 1-step recombination

IGHJ5 is a $3 \mathrm{~A}$ (7) gene with a final esebssiwaago $T_{\mathrm{Q}}$ of 0.24 (0.235 (Table 2, Additional file 3: Table S3; Table 4).

Germline functional IGHD_-_ genes for allele 1 (IGHM) 1-step recombination

IGHD2-15 is a 3 episode, 7 initial SEB and final SEB gene that begins with a mesotropic SEB. IGHD2-15 has one instance of non-contributory anisotropy. IGHD2-15 is a $3 \mathrm{M}(7) \mathrm{NCA}$ gene with a final esebssiwaago $T_{\mathrm{Q}}$ of 0.29 (0.294).

IGHD1-14 is a 3 episode, 7 initial and final SEB gene that begins with an anisotropic SEB. IGHD1-14 has two instances of anisotropy converted-to-mesotropy, and one instance of non-contributory anisotropy. IGHD1-14 is a $3 \mathrm{~A}(7) \mathrm{ACM} \times 2 \mathrm{NCA}$ with a final esebssiwaago $T_{\mathrm{Q}}$ of 0.29 (0.292).

IGHD6-13 is a 3 episode, 7 initial and final SEB gene that begins with a mesotropic SEB. IGHD6-13 has one instance of non-contributory anisotropy, and one instance of non-contributory reverse stIsotropy. IGHD613 is a $3 \mathrm{M}(7)$ NCA NCstI gene with a final esebssiwaa$g o T_{\mathrm{Q}}$ of $0.29(0.286)$ (Table 3, Additional file 4: Table S4; Table 4).

IGHD_-_-IGHJ5 genes for allele 1 (IGHM) V-to-DJ after 1-step recombination

IGHD2-15-IGHJ5 is a 3 episode, 7 initial SEB and final SEB gene that begins with a mesotropic SEB. IGHD2$15-$ IGHJ5 is a $3 \mathrm{M}(7)$ gene with a final esebssiwaago $T_{\mathrm{Q}}$ of $0.25(0.251)$.

IGHD1-14-IGHJ5 is a 3 episode, 7 initial and 3 final SEB gene that begins with a mesotropic SEB. IGHD114-IGHJ5 has one instance of anisotropy convertedto-mesotropy, and one instance of non-contributory anisotropy. IGHD1-14-IGHJ5 is a $3 \mathrm{M}$ (7) ACM NCA gene with a final esebssiwaago $T_{\mathrm{Q}}$ of $0.15(0.149)$.

IGHD6-13-IGHJ5 is a 3 episode, 7 initial and 9 final SEB gene that begins with an anisotropic SEB. IGHD6-13-IGHJ5 has one instance of anisotropy converted-to-mesotropy, and one instance of noncontributory anisotropy. IGHD6-13- IGHJ5 is a 3 A [7(+2): 9] ACM NCA gene with a final esebssiwaago $T_{\mathrm{Q}}$ of 0.20 (0.205) (Table 4, Additional file 5: Table S5).

\section{Germline functional IGHJ3, IGHJ2 and IGHJ1 genes for allele 2 (IGHD) 1-step recombination}

IGHJ3 is a $3 \mathrm{M}(7)$ gene with a final esebssiwaago $T_{\mathrm{Q}}$ of 0.11 (0.112).

IGHJ2 is a $3 \mathrm{M}(7)$ gene with a final esebssiwaago $T_{\mathrm{Q}}$ of 0.11 (0.114).

IGHJ1 is a $3 \mathrm{M}$ (7) NCA gene with a final esebssiwaa$g o T_{\mathrm{Q}}$ of $0.12(0.116)$ (Table 2, Additional file 3: Table S3; Table 5).

Germline functional IGHD_-_ genes for allele 2 (IGHD) 1-step recombination

IGHD1-26 is a 3 episode, 7 initial and final SEB gene that begins with an anisotropic SEB. IGHD1-26 has two instances of non-contributory anisotropy. IGHD1-26 is a 3 A (7) $\mathrm{NCA} \times 2$ gene with a final esebssiwaago $T_{\mathrm{Q}}$ of $0.22(0.217)$. 
IGHD6-25 is a 3 episode, 7 initial and final SEB gene that begins with an anisotropic SEB. IGHD1-14 IGHD6-25 has one instance of anisotropy converted-tomesotropy, and two instances of non-contributory anisotropy. IGHD6-25 is a $3 \mathrm{~A}(7) \mathrm{ACM} \mathrm{NCA} \times 2$ with a final esebssiwaago $T_{\mathrm{Q}} 0.17$ (0.172).

IGHD5-24/IGHD4-23/IGHD3-22 is a 3 episode, 7 initial and 5 final SEB gene that begins with an anisotropic SEB. IGHD5-24/IGHD4-23/IGHD3-22 has one instance of anisotropy converted-to-mesotropy, and one instance of non-contributory anisotropy. IGHD5-24/ IGHD4-23/IGHD3-22 is a 3 A [(7(-2): 5] ACM NCA with a final esebssiwaago $T_{\mathrm{Q}}$ of $0.22(0.216)$.

IGHD2-21 is a 3 episode, 7 initial and final SEB gene that begins with a mesotropic SEB. IGHD2-21 has one instance of non-contributory anisotropy. IGHD2-21 is a $3 \mathrm{M}$ (7) NCA with a final esebssiwaago $T_{\mathrm{Q}}$ of $0.20(0.205)$.

IGHD5-12 is a 3 episode, 7 initial SEB and final SEB gene that begins with a mesotropic SEB. IGHJ3 IGHD512 is a $3 \mathrm{M}(7)$ gene with a final esebssiwaago $T_{\mathrm{Q}}$ of 0.22 (0.218) (Table 3, Additional file 4: Table S4; Table 5).

IGHD_-_-IGHJ_ genes for allele 2 (IGHD) V-to-DJ after 1-step recombination

IGHD5-24/IGHD4-23/IGHD3-22-IGHJ1 is a 3 episode, 7 initial and final SEB gene that begins with a mesotropic SEB. IGHD5-24/IGHD4-23/IGHD3-22-IGHJ1 has one instance of indirect stIsotropy for mesotropy. IGHD5-24/ IGHD4-23/IGHD3-22-IGHJ1 is a $3 \mathrm{M}$ (7) stIMfM gene with a final esebssiwaago $T_{\mathrm{Q}}$ of $0.16(0.161)$.

IGHD1-26-IGHJ1 is a 3 episode, 7 initial SEB and final SEB gene that begins with a mesotropic SEB. IGHD1-26-IGHJ1 is a $3 \mathrm{M}(7)$ gene with a final esebssiwaago $T_{\mathrm{Q}}$ of $0.13(0.130)$.

IGHD2-21-IGHJ1 is a 3 episode, 7 initial and 6 final SEB gene that begins with a mesotropic SEB. IGHD221-IGHJ1 has two instances of non-contributory anisotropy. IGHD2-21-IGHJ1 is a $3 \mathrm{M}$ [7(-1): 6] NCA $\times 2$ gene with a final esebssiwaago $T_{\mathrm{Q}}$ of $0.21(0.206)$.

IGHD6-25-IGHJ1 is a 3 episode, 7 initial SEB and final SEB gene that begins with a mesotropic SEB. IGHD6-25-IGHJ1 is a $3 \mathrm{M}$ (7) gene with a final esebssiwaago $T_{\mathrm{Q}}$ of $0.23(0.233)$.

IGHD5-12-IGHJ2 is a 3 episode, 7 initial and 5 final SEB gene that begins with a mesotropic SEB. IGHD512-IGHJ2 has one instance of non-contributory anisotropy. IGHD5-12-IGHJ2 is a $3 \mathrm{M}$ [7(-2): 5] NCA gene with a final esebssiwaago $T_{\mathrm{Q}}$ of $0.35(0.347)$.

IGHD5-24/IGHD4-23/IGHD3-22-IGHJ3 is a 3 episode, 7 initial SEB and final SEB gene that begins with a mesotropic SEB. IGHD5-24/IGHD4-23/IGHD3-22$I G H J 3$ is a $3 \mathrm{M}(7)$ gene with a final esebssiwaago $T_{\mathrm{Q}}$ of $0.15(0.147)$.
IGHD1-26-IGHJ3 is a 3 episode, 7 initial and 6 final SEB gene that begins with a mesotropic SEB. IGHD126-IGHJ3 has one instance of non-contributory anisotropy. IGHD1-14-IGHJ5 is a $3 \mathrm{M}$ [7(-1): 6] NCA gene with a final esebssiwaago $T_{\mathrm{Q}}$ of $0.11(0.114)$.

IGHD2-21-IGHJ3 is a 3 episode, 7 initial SEB and final SEB gene that begins with a mesotropic SEB. IGHD2-21-IGHJ3 is a $3 \mathrm{M}$ (7) gene with a final esebssiwaago $_{\mathrm{Q}}$ of $0.13(0.130)$.

IGHD6-25-IGHJ3 is a 3 episode, 7 initial and 5 final SEB gene that begins with an mesotropic SEB. IGHD625-IGHJ3 has one instance of anisotropy converted-tomesotropy. IGHD6-25-IGHJ3 is a $3 \mathrm{M}$ [7(-2): 5] ACM with a final esebssiwaago $T_{\mathrm{Q}}$ of $0.20(0.195)$ (Table 5, Additional file 6: Table S6).

\section{Germline IGHV_ genes}

IGHV1-3/IGHV4-4\$ with respect to IGHJ_-IGHD_-- is a 3 episode, 7 initial and variable final SEB gene that begins with either an anisotropic SEB or a mesotropic SEB. The final esebssiwaago $T_{\mathrm{Q}}$ is variable.

IGHV3-23 is a 3 episode, 7 initial SEB and final SEB gene that begins with an anisotropic SEB. IGHV3-23 is a $3 \mathrm{~A}$ (7) gene with a final esebssiwaago $T_{\mathrm{Q}}$ of 0.33 (0.332).

IGHV4-28 is a 3 episode, 7 initial and 5 final SEB gene that begins with a mesotropic SEB. IGHV4-28 has one instance of anisotropy converted-to-mesotropy, and one instance of indirect stIsotropy for mesotropy. IGHV4-28 is a 3 M [7 (-2): 5] ACM stIMfM gene with a final esebssiwaago $T_{\mathrm{Q}}$ of $0.41(0.415)$.

IGHV3-48 is a 3 episode, 7 initial SEB and final SEB gene that begins with an anisotropic SEB. IGHV3-48 is a 3 A (7) gene with a final esebssiwaago $T_{\mathrm{Q}}$ of 0.27 (0.274).

IGHV5-51/IGHV3-53 is a 2 episode, 5 initial SEB and final SEB gene that begins with an anisotropic SEB. IGHV5-51/IGHV3-53 has one instance of noncontributory anisotropy. IGHV5-51/IGHV3-53 is a 2 A (5) NCA gene with a final esebssiwaago $T_{\mathrm{Q}}$ of 0.25 (0.245).

IGHV4-59 is a 3 episode, 7 initial SEB and final SEB gene that begins with a mesotropic SEB. IGHV4-59 is a $3 \mathrm{M}(7)$ gene with a final esebssiwaago $T_{\mathrm{Q}}$ of. 0.34 (0.336).

IGHV4-61 is a 3 episode, 7 initial SEB and final SEB gene that begins with a mesotropic SEB. IGHV4-61 is a $3 \mathrm{M}(7)$ gene with a final esebssiwaago $T_{\mathrm{Q}}$ of. 0.26 (0.258) (Table 6, Additional file 7: Table S7).

Ig heavy chain genes before iCSR and homologous recombination after IGHV1-3-IGHD_-_-IGHJ6

IGHV1-3-IGHD_-_-IGHJ6 is a 3 episode, 7 initial and 9* final SEB gene that begins with a mesotropic SEB. IGHV1-3- IGHD_-_IGHJ6 has one instance of 
anisotropy converted-to-mesotropy, and one instance of indirect stIsotropy for anisotropy. IGHV1-3- IGHD_-IGHJ6 is a $3 \mathrm{M}\left[7(+2)(+1): 9^{*}\right]$ ACM stIMfA* gene with a final esebssiwaago $T_{\mathrm{Q}} 0.23(0.226)$.

MIR4537 with respect to V1-3- D_-_-J6 is a 3 episode, 7 initial and final SEB gene that begins with an anisotropic SEB. MIR4537 with respect to V1-3-D_-_-J6 is a 3 A (7) gene with a final esebssiwaago $T_{\mathrm{Q}} 0.25(0.251)$.

MIR4507/MIR4538 with respect to V1-3-D_-_-J6 is a 3 episode, 7 initial and final SEB gene that begins with a mesotropic SEB. MIR4507/MIR4538 with respect to $V 1-3-D_{-}--J 6$ is a $3 \mathrm{M}(7)$ gene with a final esebssiwaa$g o T_{\mathrm{Q}}$ of $0.26(0.260)$.

MIR4539 with respect to V1-3-D_-_-J6 is a 3 episode, 7 initial and final SEB gene that begins with a mesotropic SEB. MIR4539 with respect to V1-3-D_--J6 is a $3 \mathrm{M}(7)$ gene with a final esebssiwaago $T_{\mathrm{Q}}$ of $0.27(0.268)$.

IGHD with respect to V1-3- D_-_-J6 is a 3 episode, 7 initial and final SEB gene that begins with an anisotropic SEB. IGHD with respect to V1-3-D_-_-J6 is a 3 A (7) gene with a final esebssiwaago $T_{\mathrm{Q}}$ of 0.20 (0.198) (Table 7, Additional file 8: Table S8).

Ig heavy chain genes after iCSR, homologous recombination and further CSRs after IGHV1-3-IGHD_-_IGHJ6

V1-3-D__-J6-IGHM is a 2 episode, 5 initial and final SEB gene that begins with a mesotropic SEB. V1-3-D_-_-J6-IGHM is a $2 \mathrm{M}$ (5) gene with a final esebssiwaago $T_{\mathrm{Q}}$ of 0.27 (0.275).

V1-3-D_-_-IGHD is a 2 episode, 5 initial and final SEB gene that begins with a mesotropic SEB. V1-3D_-_IGHD is a $2 \mathrm{M}(5)$ gene with a final esebssiwaa$g o T_{\mathrm{Q}}$ of 0.32 (0.320).

V1-3-D_-_-IGHG3 is a 2 episode, 5 initial and 4 final SEB gene that begins with a mesotropic SEB.
V1-3-D_-_-IGHG3 has one instance of anisotropy converted-to-mesotropy. $V 1-3-D_{-}-{ }_{-}-I G H G 3$ is a $2 \mathrm{M}$ [5(-1): 4] ACM gene with a final esebssiwaago $T_{\mathrm{Q}}$ of 0.31 (0.306).

V1-3-D_-_-IGHG4 is a 2 episode, 5 initial and 5* final SEB gene that begins with an anisotropic SEB. V1-3-D_-_-IGHG4 has one instance of anisotropy converted-to-mesotropy. $V 1-3-D_{-}-I_{-I G G 4}$ is $2 \mathrm{~A}$ [5(+1): 5] ACM $^{*}$ gene with a final esebssiwaago $T_{\mathrm{Q}}$ of 0.24 (0.237).

V1-3-D__-IGHA2 is a 2 episode, 5 initial and 7 final SEB gene that begins with a mesotropic SEB. V1-3-D_-_-IGHA2 has one instance of anisotropy converted-to-mesotropy. V1-3-D_-- IGHA2 s a $3 \mathrm{M}$ [5(+2): 7] ACM gene with a final esebssiwaago $T_{\mathrm{Q}}$ of 0.18 (0.185) (Table 7, Additional file 8: Table S8).

See Table 7 and Additional file 8: Table S8 for with respect to V1-3-D_-_IGHM, with respect to V1-3D__-IGHD, with respect to V1-3-D__-IGHG3, with respect to V1-3-D_-_-IGHG4, and with respect to V13-D_-_-IGHA2 genes.

Ig heavy chain genes before iCSR and initial allelic exclusion after IGHV3-23-IGHD_-_-IGHJ6

IGHV3-23- IGHD_-_IGHJ6 is a 3 episode, 7 initial and final SEB gene that begins with a mesotropic SEB. IGHV3-23- IGHD_-_-IGHJ6 is a $3 \mathrm{M}$ (7) gene with a final esebssiwaago $T_{\mathrm{Q}}$ of 0.29 (0.285).

MIR4537 with respect to V3-23-D_-_J6 is a 3 episode, 7 initial and final SEB gene that begins with an anisotropic SEB. MIR4537 with respect to V3-23-D_-_-J6 is a $3 \mathrm{~A}(7)$ gene with a final esebssiwaago $T_{\mathrm{Q}}$ of 0.27 (0.272).

MIR4507/MIR4538 with respect to V3-23-D_-_-J6 is a 3 episode, 7 initial and final SEB gene that begins with

Table 6 Chromosome $14(-)$ strand chromatin Ig heavy chain locus variability gene esebssiwaagot $s$ for germline genes in native 5'- > 3' chronology

\begin{tabular}{|c|c|c|c|c|}
\hline Germline Gene $e^{1 a, b}$ & Germline gene locus ${ }^{1 a}$ & $\begin{array}{l}\text { Total no. of } \\
\text { transcribed bases } \\
\text { at gene locus or n/a } \\
\text { (episode category) }\end{array}$ & $\begin{array}{l}\text { Initial no. of } \\
\text { sub-episode blocks } \\
\text { (converted final no. } \\
\text { of sub-episode } \\
\text { blocks, or n/a) }\end{array}$ & $\begin{array}{l}\text { Germline gene } \\
\text { 2-digit (3-digit) } \\
\text { esebssiwaagoT, } \\
\text { or n/a }\end{array}$ \\
\hline $\begin{array}{l}\text { IGHV1-3 \& } \\
\text { IGHV4-4 }\end{array}$ & Inc-AL901608.1-10//GHV1-3/IGHV4-4 & $10,439(3)$ & 7 (varies) & varies \\
\hline IGHV3-23 & IGHV3-23 & $535(3)$ & 7 (n/a) & $0.33(0.332)$ \\
\hline IGHV4-28 & IGHV4-28 & $507(3)$ & $7(\mathrm{n} / \mathrm{a})$ & $0.41(0.415)$ \\
\hline IGHV3-48 & IGHV3-48 & $535(3)$ & $7(\mathrm{n} / \mathrm{a})$ & $0.27(0.274)$ \\
\hline $\begin{array}{l}\text { IGHV5-51\& } \\
\text { IGHV3-53 }\end{array}$ & $\begin{array}{l}\text { GC14M107956/Inc-AL901608.1-17 \{IGHV5-51/IGHVIII-51-1 (pseudo- } \\
\text { gene)/ IGHVII-51-2 (pseudogene)/ IGHV3-52 (pseudogene)/ IGHV3-53 }\end{array}$ & $23,464(2)$ & $7(5)$ & $0.25(0.245)$ \\
\hline IGHV4-59 & IGHV4-59 & $577(3)$ & $7(\mathrm{n} / \mathrm{a})$ & $0.34(0.336)$ \\
\hline IGHV4-61 & IGHV4-61 & $539(3)$ & 7 (n/a) & $0.26(0.258)$ \\
\hline
\end{tabular}

${ }^{1 a}$ only lg genes and gene loci included, non-coding RNA genes (processed pseudogenes) excluded ${ }^{\text {b }}$ sample set of variability genes. ${ }^{2}>11,864 \leq 265,005$ total transcribed bases, Episode category 2 gene; Episode category 3 gene bases, $\leq 11,864$ total transcribed bases, Episode category 3 gene. ${ }^{\S} / n c-A L 901608.1-10 / / G H V 1-$ 3/IGHV4-4 gene locus esebssiwaago $T_{\mathrm{Q}}$ is $D_{-}$-_ $_{-} \mathrm{J}_{-}$location dependent 


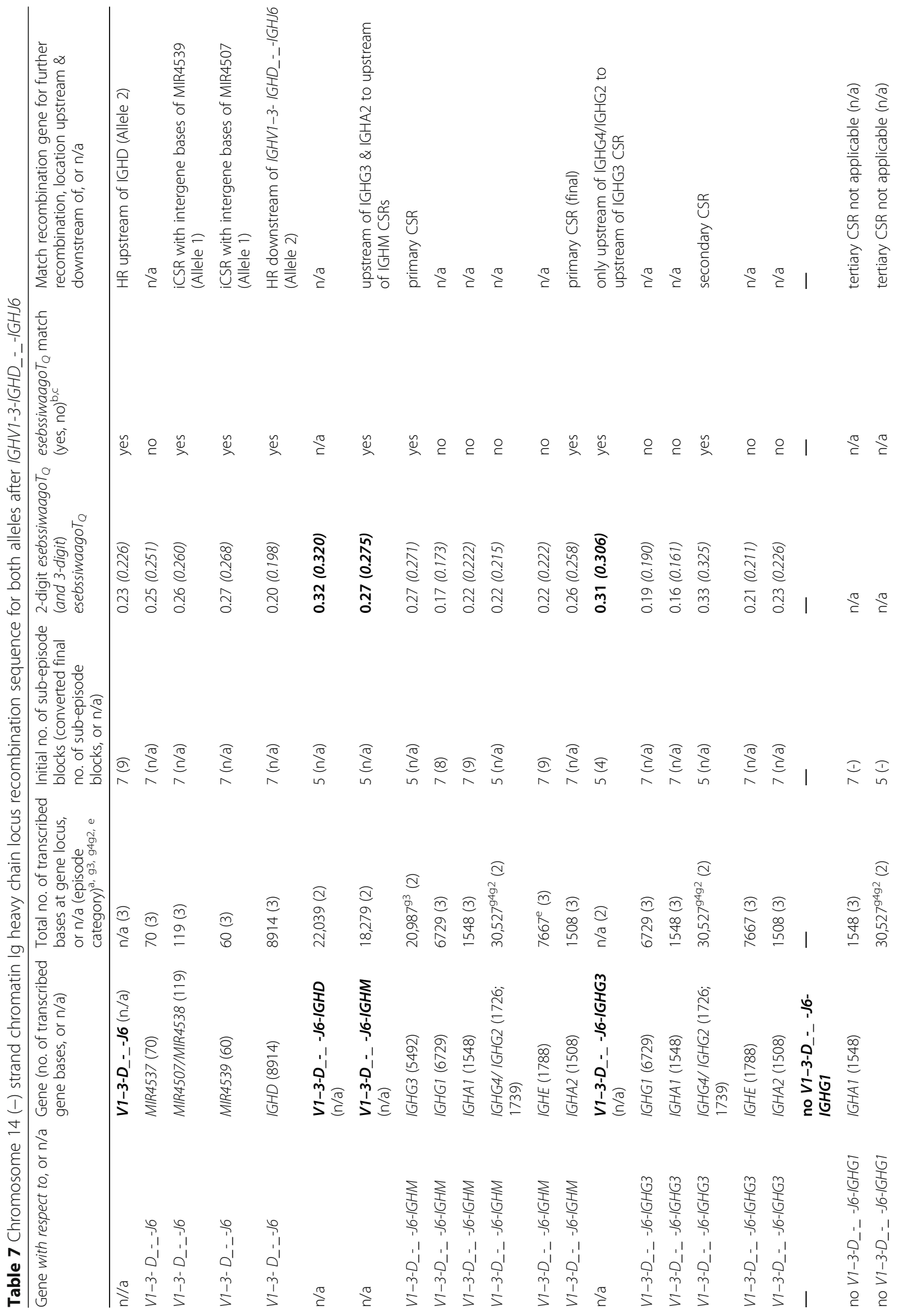




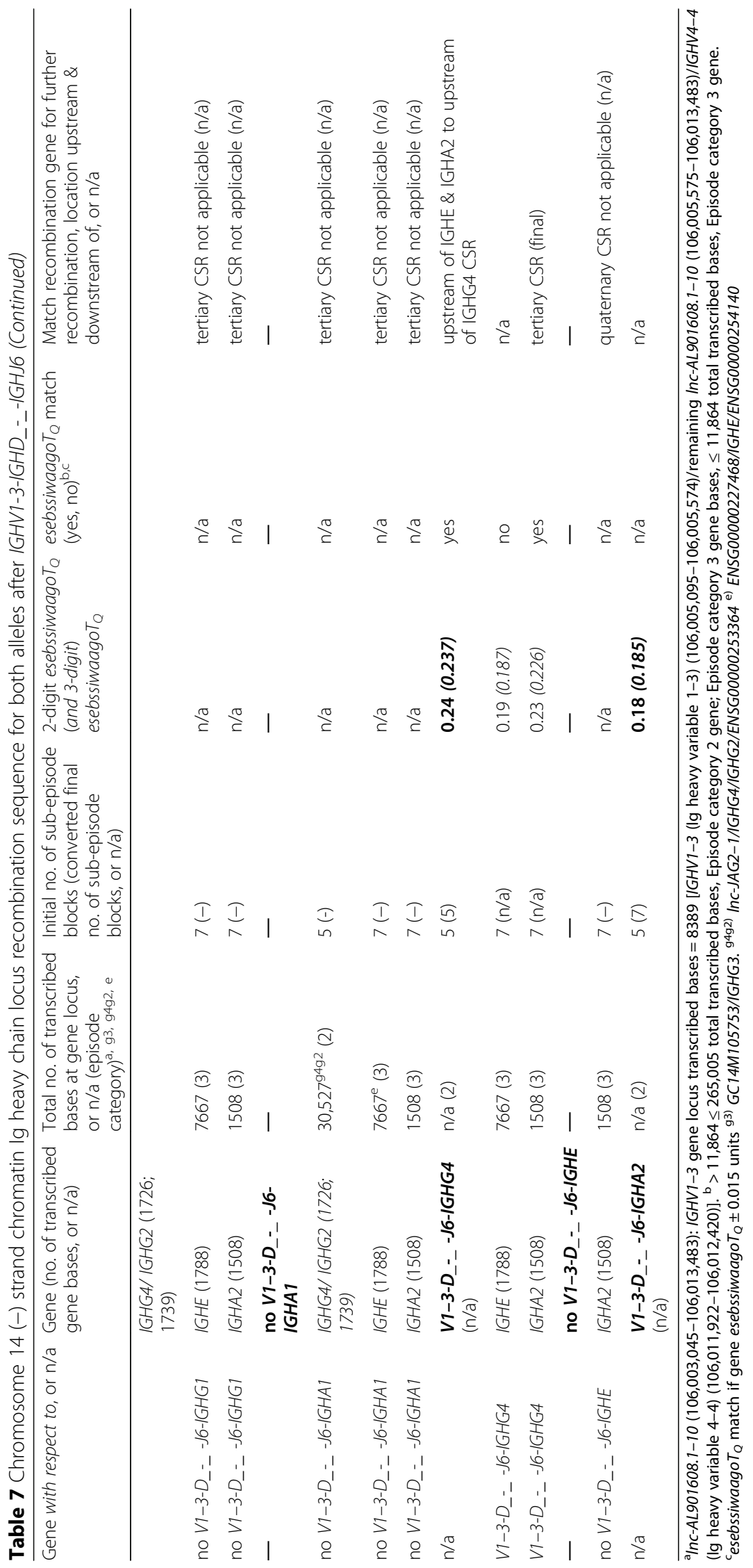


a mesotropic SEB. MIR4507/MIR4538 with respect to V3-23-D_--J6 is a $3 \mathrm{M}(7)$ gene with a final esebssiwaa$g o T_{\mathrm{Q}}$ of $0.28(0.277)$.

MIR4539 with respect to V3-23-D_-_-J6 is a 3 episode, 7 initial and 5 final SEB gene that begins with a mesotropic SEB. MIR4539 with respect to V3-23$D_{-}-$-J6 has one instance of anisotropy converted-tomesotropy. MIR4539 with respect to V3-23-D_-_-J6 is a $3 \mathrm{M}$ [7(-2): 5] ACM gene with a final esebssiwaago $_{\mathrm{Q}}$ of $0.23(0.232)$.

IGHD with respect to V3-23-D_-_-J6 is a 3 episode, 7 initial and 8 final SEB gene that begins with an anisotropic SEB. IGHD with respect to V3-23-D_-_J6 has one instance of anisotropy converted-to-mesotropy. IGHD with respect to V3-23-D_-_-J6 is a $3 \mathrm{~A}$ [7 (+1): 8] ACM 3 A (7) gene with a final esebssiwaago $T_{\mathrm{Q}}$ of 0.22 (0.218) (Table 8, Additional file 9: Table S9).

\section{Ig heavy chain genes after iCSR and further CSRs}

following IGHV3-23-IGHD_-_IGHJ6

V3-23-D_-_-J6-IGHM is a 3 episode, 7 initial and final SEB gene that begins with a mesotropic SEB. V3-23-D_-_-J6-IGHM is a $3 \mathrm{M}$ (7) gene with a final esebssiwaago $T_{\mathrm{Q}}$ of. 0.28 (0.277).

V3-23-D_-_-J6-IGHG1 is a 2 episode, 5 initial and final SEB gene that begins with a mesotropic SEB. V323-D_-_J6-IGHG1 has one instance of anisotropy converted-to-mesotropy. V3-23-D_-_-J6-IGHG1 is $2 \mathrm{M}$ (5) $\mathrm{ACM}$ gene with a final esebssiwaago $T_{\mathrm{Q}}$ of 0.26 (0.256).

V3-23-D_-_J6-IGHA1 is a 3 episode, 7 initial and final SEB gene that begins with a mesotropic SEB. V323-D_-_J6-IGHA1 has one instance of anisotropy converted-to-mesotropy. V3-23-D_-_-J6-IGHA1 is $3 \mathrm{M}$ (7) $\mathrm{ACM}$ gene with a final esebssiwaago $T_{\mathrm{Q}}$ of 0.17 (0.171).

V3-23-D_-_J6-IGHE is a 3 episode, 7 initial and final SEB gene that begins with a mesotropic SEB. V3-23-D_-_-J6-IGHE is a $3 \mathrm{M}(7))$ gene with a final esebssiwaago $T_{\mathrm{Q}}$ of. 0.21 (0.205).

V3-23-D_-_J6-IGHA2 is a 2 episode, 5 initial and 7 final SEB gene that begins with an anisotropic SEB. V3-23D_-_J6-IGHA2 s a $3 \mathrm{~A}$ (7) gene with a final esebssiwaa$g o T_{\mathrm{Q}}$ of 0.21 (0.208) (Table 8, Additional file 9: Table S9).

See Table 8 and Additional file 9: Table S9 for with respect to V3-23-D_-_J6-IGHM, with respect to V3-23D_-_J6-IGHG1, with respect to V3-23-D_-_J6-IGHA1, with respect to V3-23-D_-_J6-IGHE, and with respect to V3-23-D_-_J6-IGHA2 genes.

Ig heavy chain genes before iCSR and initial allelic exclusion after IGHV5-51-IGHD_--IGHJ6

IGHV5-51-IGHD_-_IGHJ6 is a 2 episode, 5 initial and final SEB gene that begins with a mesotropic SEB.
IGHV5-51-IGHD_-_-IGHJ6 is a $2 \mathrm{M}$ (5) gene with a final esebssiwaago $T_{\mathrm{Q}}$ of $0.23(0.233)$.

MIR4537 with respect to V5-51-D_-_-J6 is a 3 episode, 7 initial and final SEB gene that begins with a mesotropic SEB. MIR4537 with respect to V5-51$D_{-}--$J6 is a $3 \mathrm{M}(7)$ gene with a final esebssiwaago $T_{\mathrm{Q}}$ of 0.15 (0.152).

MIR4507/MIR4538 with respect to V5-51-D_-_-J6 is a 3 episode, 7 initial and final SEB gene that begins with an anisotropic SEB. MIR4507/MIR4538 with respect to V5-51-D_-_-J6 is a $3 \mathrm{~A}(7)$ gene with a final esebssiwaagot $T_{\mathrm{Q}}$ of $0.15(0.155)$.

MIR4539 with respect to V5-51-D_-_J6 is a 3 episode, 7 initial and final SEB gene that begins with a mesotropic SEB. MIR4539 with respect to V5-51-D_-_-J6 is a $3 \mathrm{M}$ (7) gene with a final esebssiwaago $T_{\mathrm{Q}}$ of $0.16(0.159)$.

IGHD with respect to V5-51-D_-_-J6 is a 3 episode, 7 initial and 9 final SEB gene that begins with an anisotropic SEB. IGHD with respect to V5-51-D_-_J6 has one instance of anisotropy converted-to-mesotropy. IGHD with respect to V3-51-D_-_-J6 is a 3 A [7(+2): 9] ACM gene with a final esebssiwaago $T_{\mathrm{Q}}$ of $0.17(0.169)$ (Table 9, Additional file 10: Table S10).

\section{Ig heavy chain genes after iCSR and further CSRs following IGHV5-51-IGHD_-_IGHJ6}

V5-51-D_-_-J6-IGHM is a 2 episode, 5 initial and final SEB gene that begins with an anisotropic SEB. V5-51D_-_-J6-IGHM is a 2 A (5) gene with a final esebssiwaa$g o T_{\mathrm{Q}}$ of. $0.16(0.165)$.

V5-51-D_-_J6-IGHG1 is a 2 episode, 5 initial and final SEB gene that begins with a mesotropic SEB. V551-D_-_-J6-IGHG1 has one instance of anisotropy converted-to-mesotropy. V5-51-D_-_-J6-IGHG1 is $2 \mathrm{M}$ (5) $\mathrm{ACM}$ gene with a final esebssiwaago $T_{\mathrm{Q}}$ of 0.15 (0.153).

V5-51-D_-_J6-IGHA1 is a is a 2 episode, 5 initial and final SEB gene that begins with a mesotropic SEB. V5-51-D__-J6-IGHA1 is $2 \mathrm{M}(5)$ gene with a final esebssiwaago $T_{\mathrm{Q}}$ of $0.13(0.134)$.

V5-51-D_-_-J6-IGHG4 is a is a 2 episode, 5 initial and final SEB gene that begins with a mesotropic SEB. V5-51-D_-_J6-IGHG4 is a $2 \mathrm{M}$ (5) gene with a final esebssiwaago $T_{\mathrm{Q}}$ of $0.18(0.184)$.

V5-51-D_-_J6-IGHE is a 2 episode, 5 initial and 6* final SEB gene that begins with an anisotropic SEB. V551-D_-_J6-IGHE has one instance of anisotropy converted-to-mesotropy. V5-51-D_-_J6-IGHE is $2 \mathrm{~A}$ $\left[5(+1): 6^{*}\right] \mathrm{ACM}^{*}$ gene with a final esebssiwaago $T_{\mathrm{Q}}$ of $0.15(0.152)$.

V5-51-D_-J6-IGHA2 is a 2 episode, 5 initial and final SEB gene that begins with a mesotropic SEB. V551-D_-_-J_-J6-IGHA2 has one instance of indirect stIsotropy for anisotropy. V5-51-D_-_-J6-IGHA2 s a $2 \mathrm{M}$ (5) 


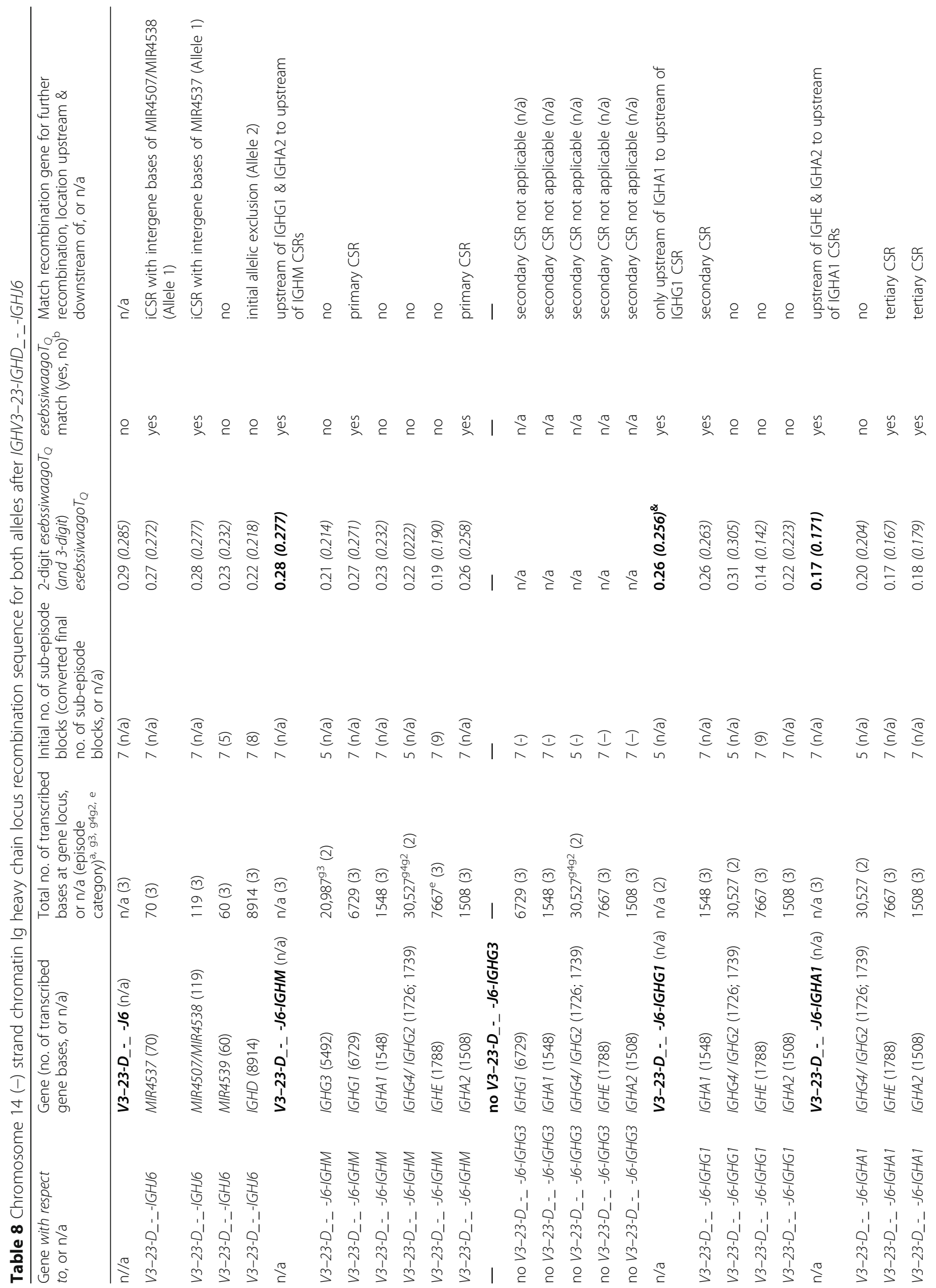




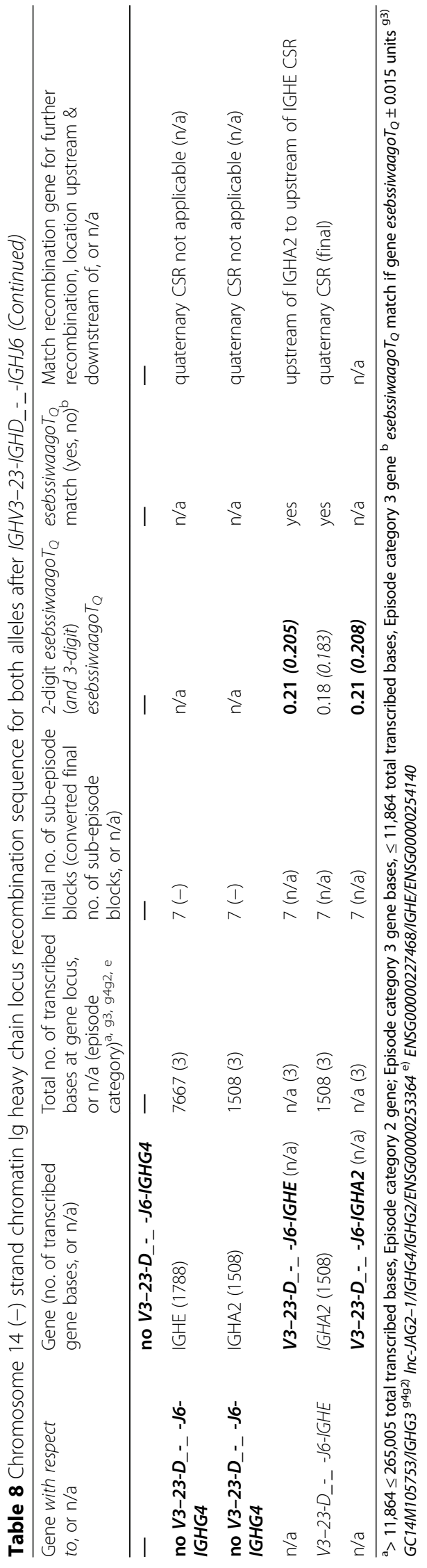




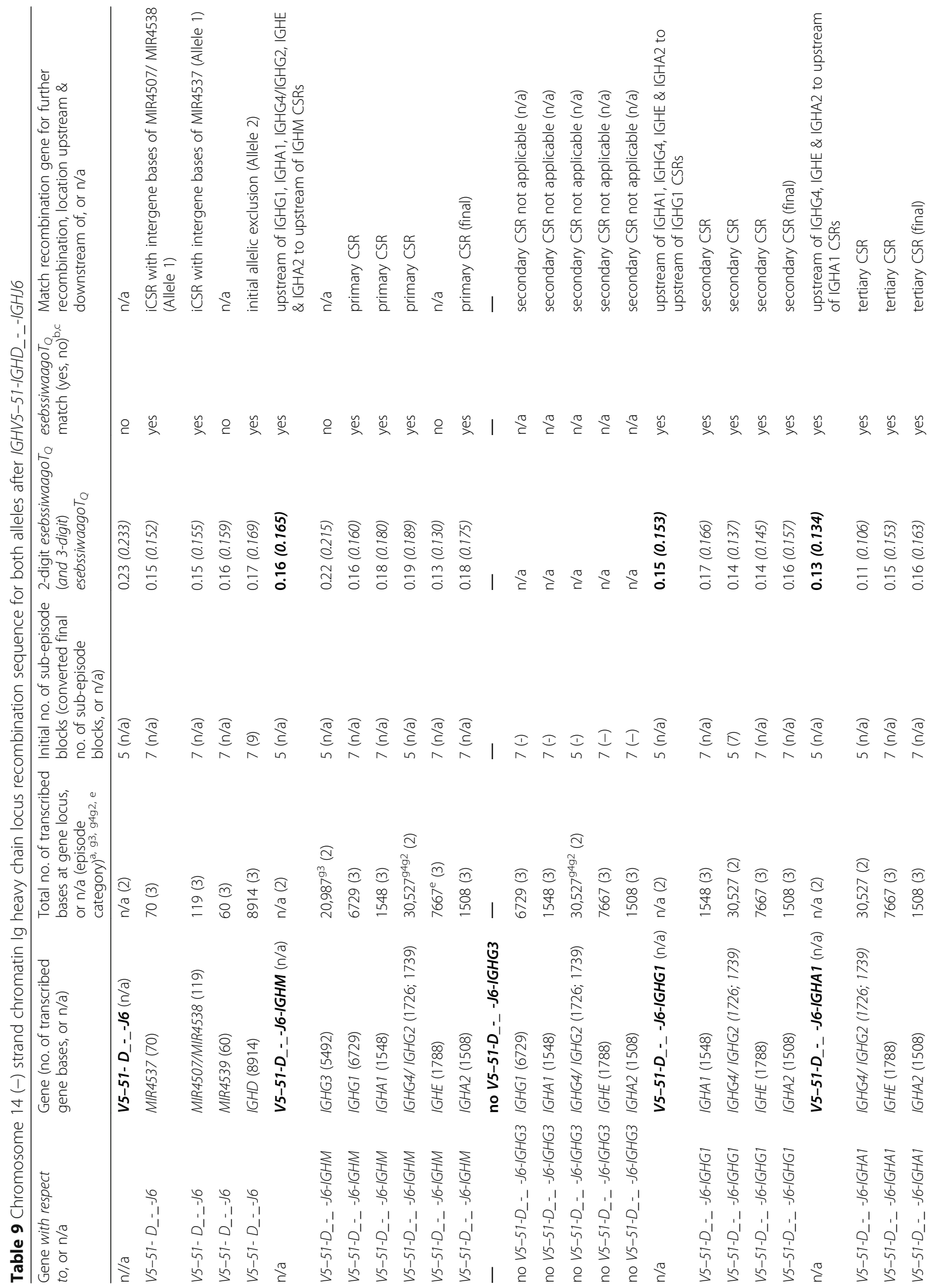




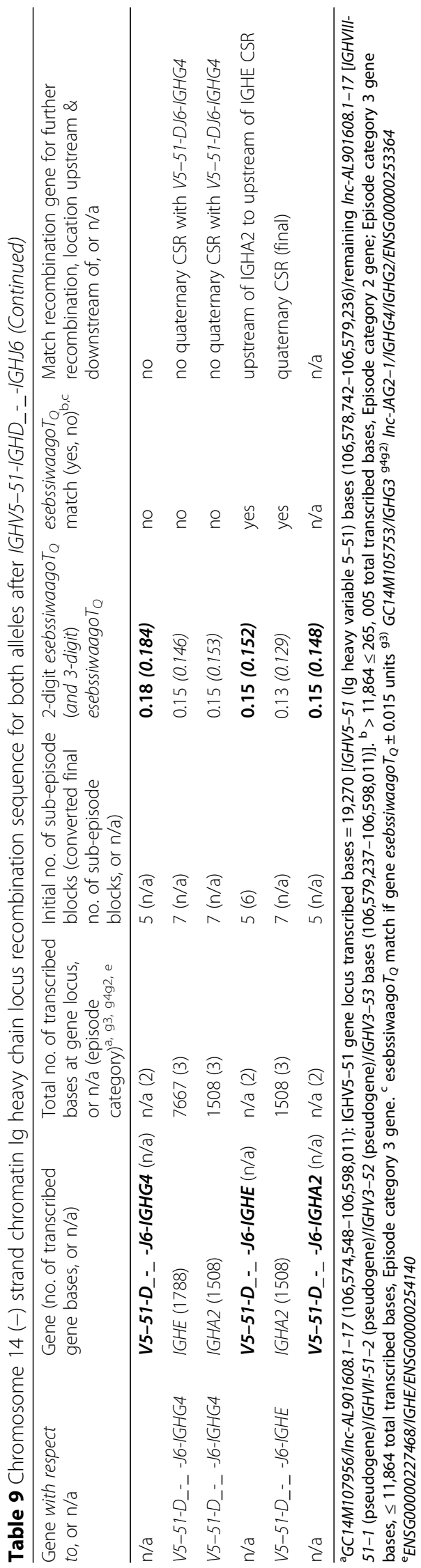


stIMfA gene with a final esebssiwaago $T_{\mathrm{Q}}$ of $0.15(0.148)$ (Table 9, Additional file 10: Table S10).

See Table 9 and Additional file 10: Table S10 for with respect to V5-51-D_-_-J6-IGHM, with respect to V5-51D_-_J6-IGHG1, with respect to V5-51-D_-_-J6IGHA1, with respect to V5-51-D_-_J6-IGHG4, with respect to V5-51-D_-_-J6-IGHE, and with respect to V5-51-D_-_-J6-IGHA2 genes.

\section{Discussion}

The intracellular pressure required to establish a horizontal reading frame for recombination of joining and diversity genes in native germline arrangement is the basis for predictable gene rearrangement

Variability-to-diversity-to-joining gene recombination is close to perfect when it completes in the pressuromodulated state in vivo [18]. As true allelic exclusion of the non-classical pathway in every case is due to failure of Allele 2 (IGHD) homologous recombination and not a failure of VDJ, Allele 1 (IGHM) accounts for $50 \%$ of VDJs while Allele 2 (IGHD) accounts for the other 50\% of VDJs in vivo. The frequencies of diversity (D), joining $(\mathrm{J})$ and variability $(\mathrm{V})$ gene distribution is known, that of IGHJ6 is $40 \%$ (esebssiwaago $T_{\mathrm{Q}}: 0.097$ ), that of IGHJ5 is $10 \%$ (esebssiwaago $T_{\mathrm{Q}}: 0.235$ ), that of IGHJ4 is $32 \%$ (esebssiwaago $T_{\mathrm{Q}}: 0.110$ ), that of $I G H J 3$ is $\sim 8.5 \%$ (esebssiwaago $T_{\mathrm{Q}}: 0.112$ ), that of $I G H J 2$ is $\sim 1.5 \%$ (esebssiwaa$\left.g o T_{\mathrm{Q}}: \quad 0.114\right)$ and IGHJ1 accounts for $\sim 8.5 \%$ (esebssiwaago $T_{\mathrm{Q}}$ : 0.116).

Allele 1 (IGHM) gene recombination begins first, when B-cell intracellular pressure is in the suprapressuromodulated gene expression range [4]. Only IGHJ5 that expresses at an esebssiwaago $T_{\mathrm{Q}}$ of 0.235 units can be the $1^{\text {st }}$ step candidate gene for Allele 1, which only leaves 1 other step for Allele $1 \mathrm{~J} \leftrightarrow \mathrm{D}$ recombination. Thus, IGHJ5 is a 1 -step J_ gene and recipient of $10 \%$ of Allele 1 one-step $\mathrm{D}_{-}$-_ genes $10 \%$ of the time, while it is a 2-step stepping stone $J_{\text {_ }}$ gene for Allele 1 non-functional (nf) gene IGHD1-20 (nf) (esebssiwaa$\left.g o T_{\mathrm{Q}}: 0.406\right)$ the rest of the time, making IGHJ6 the recipient gene for $40 \%$ of the $\mathrm{D}_{-}-$- genes involved in the 2-step of Allele 1 two-step.

Allele 2 (IGHD) gene recombination follows that of Allele 1, when B-cell intracellular pressure is in the infra-pressuromodulated range, IGHJ1 through IGHJ4 all express in the 0.110 to 0.116 esebssiwaago $T_{\mathrm{Q}}$ units range; of these, the first $\mathrm{J}_{-}$gene IGHJ1 and the third one IGHJ3 are each present at a frequency of $\sim 8.5 \%$ and are Allele 2 1-step J_ genes. IGHJ2 is present at a frequency of $\sim 1.5 \%$ and is also an Allele 2 1-step J_ gene. As IGHJ2 is present at the lowest frequency, this implies that it is the 2-step stepping stone $J_{-}$gene for Allele 2 non-functional genes IGHD4-11 (nf) (esebssiwaago $T_{\mathrm{Q}}$ : 0.293 ) and IGHD5-18 (nf) (esebssiwaago $T_{\mathrm{Q}}: 0.254$ ) the rest of the time, making IGHJ2 the recipient of $32 \%$ of Allele 2 two-step D_-_ genes.

Germline functional diversity genes in their native configuration and destined to participate in 2-step Allele 1 (IGHM) recombination have esebssiwaago $T_{\mathrm{Q}} \mathrm{S}$ in the 0.342 to 0.295 units range, which is approximately $40 \%$ of $\mathrm{D}_{-}$-- genes, while those germline functional diversity genes that participate in 1-step Allele 1 recombination have esebssiwaago $T_{\mathrm{Q}} \mathrm{S}$ in the 0.294 to 0.286 units range, which is the remaining $10 \%$ of $\mathrm{D}_{-}-$- genes.

Germline functional diversity genes in their native configuration and destined to participate in 2-step Allele 2 (IGHD) recombination have esebssiwaago $T_{Q} \mathrm{~S}$ in the 0.276 to 0.233 units range, which is approximately $32 \%$ of $\mathrm{D}_{\text {_-_ }}$ genes, while those germline functional diversity genes that participate in 1-step Allele 2 recombination have esebssiwaago $T_{\mathrm{Q}}$ in the 0.218 to 0.172 units range, which is the remaining $18 \%$ of $\mathrm{D}_{-}-{ }_{-}$genes.

Germline non-functional diversity gene IGHD7-27 that does not participate in $\mathrm{D}$ to $\mathrm{J}$ recombination has an esebssiwaago $T_{\mathrm{Q}}$ of 0.165 .

Therefore, the intracellular pressure required to establish a horizontal reading frame for efficient RAG1 and RAG2 recombinase activity [4] is the basis for predictable $\mathrm{B}$-cell joining and diversity gene recombination in the pressuromodulated state in vivo, of which the gene esebssiwaagot $_{Q}$ is the measure as it is a property of the gene.

The 2-step and 1-step $D$ to $\mathrm{J}$ recombination processes are mutually exclusive

The 2-step Allele 1 (IGHM) gene recombination process involves a more primed CD4R+ CD40LG T-cell-mediated CD40R B-cell CM polarization pressuromodulation effect as an uphill intracellular pressure of 0.41 esebssiwaago $T_{\mathrm{Q}}$ units is required for IGHJ5 (esebssiwaago $T_{\mathrm{Q}}: 0.235$ ) to IGHD1-20 (nf) (esebssiwaago $\left.T_{\mathrm{Q}}: 0.406\right)$ gene recombination $(\mathrm{J5} \longrightarrow \mathrm{D1}-20(\mathrm{nf}))$.

The 1-step Allele 1 recombination process involves a less primed CD4R+ CD40LG T-cell-mediated CD40R B-cell $\mathrm{CM}$ polarization pressuromodulation effect when an intracellular pressure of 0.36 units is achieved, which is sufficient for PRDM1 expression at 0.36 units and B-cell cyclic pressure oscillation, however Allele 1 two-step does not take place. Instead, 1-step Allele 1 recombination takes place as an intracellular pressure of 0.30 esebssiwaago $T_{\mathrm{Q}}$ units is achieved when IGHJ5 (esebssiwaago $T_{\mathrm{Q}}: 0.235$ ) to IGHD2-1 (esebssiwagoo $T_{\mathrm{Q}}$ : 0.296) recombination takes place $(J 5 \longrightarrow D 2-1)$. The 2-step Allele 1 (IGHD) gene recombination process can also take place as it requires a maximum intracellular pressure of 0.293 esebssiwaago $T_{\mathrm{Q}}$ units to engage IGHD4-11 (nf) for recombination with IGHJ2 (esebssiwaago $\left.T_{\mathrm{Q}}: 0.114\right)(D 4-11(\mathrm{nf}) \longrightarrow J 2)$.

Therefore, when the Allele 1 (IGHM) recombination process is a 2-step process $[\mathrm{J} \rightarrow \mathrm{D}(\mathrm{D} \rightarrow \mathrm{J})]$, then the 
Allele 2 (IGHD) recombination process is a 1-step process $(\mathrm{D} \rightarrow \mathrm{J})$; and when the Allele 1 (IGHM) recombination process is a 1-step process $(J \rightarrow D)$, then the Allele 2 (IGHD) recombination process is a 2-step process $[\mathrm{D} \rightarrow \mathrm{J}(\mathrm{D} \rightarrow \mathrm{J})]$.

The above deductions are supported by the literature as D_-_ gene, IGHD4-17, must be the Allele 1 (IGHM) Step $2 \mathrm{~b}$ of 2 recombination gene in which case the $\mathrm{D}_{-}-$ gene, IGHD1-26, must be the Allele 2 (IGHD) Step 1 of 1 recombination gene [20].

Allele 1 (IGHM) 2-step recombination involves germline IGHJ5 and IGHD1-20 (nf) recombination (step 1), IGHD_-_ and IGHJ6 gene with respect to D1-20 (nf)-J5 recombination (step $2 \mathrm{a}$ ) that results in IGHD_-_-IGHJ6 (step 2b) and then IGHV_-_ to IGHD_-_IGHJ6 recombination that results in $V_{-}-{ }_{-}-D J 6$

Step 1 of the Allele 1 two-step recombination process begins after PRDM1 expression at 0.36 esebssiwaago $T_{\mathrm{Q}}$ units, when B-cell intracellular pressure decreases to just below the $C D 40$ gene expression intracellular pressure of 0.26 esebssiwaago $T_{\mathrm{Q}}$ units to around 0.24 units and RAG2 engages the upstream handle of IGHJ5 at an esebssiwaago $T_{\mathrm{Q}}$ of 0.235 units. At around 0.24 units there is an increase in cell pressure back to around 0.26 units as the PRDM1 effect wanes, when the 1st maximum polarization period begins with increasing cell pressure to 0.41 units when RAG2 engages the downstream handle of IGHD1-20 (nf), which results in IGHD1-20 (nf)-IGHJ5 (Fig. 1).

The step 2a of the process is downhill and begins after RAG2 engages IGHD3-10 with respect to D1-20 (nf)-J5, which is the upper limit $\mathrm{D}_{-}-$- gene with respect to D120 (nf)-J5 with an esebssiwaago $T_{\mathrm{Q}}$ of 0.402 units. Then, cell pressure decreases back down into the PRDM1 maximum expression range at 0.36 units, and thereafter, at a sufficient enough rate through the CD40 expression cell pressure of 0.26 units into peri-nadir at 0.15 plus minus 0.05 units, which defines four fifths of one limb of $1^{\text {st }}$ fully refractory period. In the downhill limb of the fully refractory period, RAG1 engages IGHD4-17 with respect to D1-20 (nf)-J5 at 0.15 (0.150) units, which is lower limit for D_-_ genes with respect to D1-20 (nf)-J5. The step 2a stage completes after RAG1 association with IGHJ6 with respect to D1-20-J5 at 0.101 units of the period nadir, when the two free ends of DNA come together to result in IGHD___-IGHJ6 (step 2b) (Fig. 1).

The IGHD__-IGHJ6 recombined gene esebssiwaago $T_{\mathrm{Q}}$ range is $0.13(0.133)$ to $0.30(0.297)$ units and the $I G H V_{-}$- gene esebssiwaago $T_{\mathrm{Q}}$ range with respect to IGHD__-IGHJ6 is an unestablished lower limit to 0.41 (0.415) units. The VDJ step of the process completes between the $1^{\text {st }}$ fully refractory pre-nadir period and the uphill limb of $2^{\text {nd }}$ maximum polarization period. The lower limit of the $I G H V_{-}$gene esebssiwaago $T_{\mathrm{Q}}$ range is unestablished as more variability genes need to be sampled (Fig. 1).

Allele 1 recombination is a 2 -step process when a more primed CD4R+ T-cell is involved and the greater magnitude of the B-cell polarization pressuromodulation effect. This implies that during a more robust pressuromodulation effect:

(1) there is deceleration during the 2 -step Allele 1 step 1 downhill limb around an intracellular pressure of 0.25 esebssiwaago $T_{\mathrm{Q}}$ units because it is a function of PRDM1 expression that results in full expression of $C D 40$ at 0.26 units, after which there is maximum B-cell polarization and acceleration in the opposite direction with an uphill increase in cell pressure back to 0.41 units, then down into the PRDM1 expression pressure of 0.36 units;

and (2) there is acceleration during the step 2a downhill limb through the CD40 expression intracellular pressure into the fully refractory period because it is a function of preceding maximum CD40 expression followed by PRDM1 expression in series that then results in nonexpression of $C D 40$ at 0.26 units, after which there is maintained acceleration downhill into the peri-nadir due to the PRDM1 C-MYC gene antagonism effect.

\section{Allele 2 (IGHD) 1-step recombination step involves germline IGHD_-_ and IGHJ1, IGHJ2 or IGHJ3 recombin- ation that results in IGHD_-_-IGHJ1 -J2 or -J3 (step1) and then IGHV_-_ to IGHD_-_J1-J4, IGHD_-_-J2-J4 or IGHD_- _-J3-J4 recombination that results in $V_{-}-_{-}-D J 6$}

Step 1 of the Allele 2 one-step recombination process begins during the downhill limb of the $1^{\text {st }}$ refractory period in between $0.22(0.218)$ and 0.17 (0.172) esebssiwaggo $_{\mathrm{Q}}$ units at which the $I G H D_{-}$-_ genes are substrates for RAG. Step 1 completes to the point of $I G H D_{-}-I_{-} G H J 1,-J 2$ or -J3 during peri-nadir before the uphill limb in between $0.12(0.116)$ and $0.11(0.112)$ units at which the 1-step IGHJ_ genes, IGHJ1 ( 8.5\%), IGHJ2 $(\sim 1.5 \%)$ or IGHJ3 $(\sim 8.5 \%)$ are substrates for the same (Fig. 2).

The step 1 IGHD__-IGHJ1, -J2 or -J3 recombined gene esebssiwaago $T_{\mathrm{Q}}$ range is $0.11(0.114)$ to $0.35(0.347)$ units and the IGHV_-_ gene with respect to IGHD_-_IGHJ12, $-J$ and $-J 3$ esebssiwaago $T_{\mathrm{Q}}$ range is an unestablished lower limit to $0.41(0.415)$ units. The VDJ step of the Allele 2 one-step process completes during the uphill limb of the $1^{\text {st }}$ fully refractory period into the $2^{\text {nd }}$ maximum polarization period (Fig. 2).

The Allele 2 recombination process is a 1-step process during the more primed CD4R+ T-cell-mediated polarization effect and follows Allele 2 two-step after CD40 non-expression as Allele 1 one-step begins during the downhill limb of the $1^{\text {st }}$ fully refractory period. 

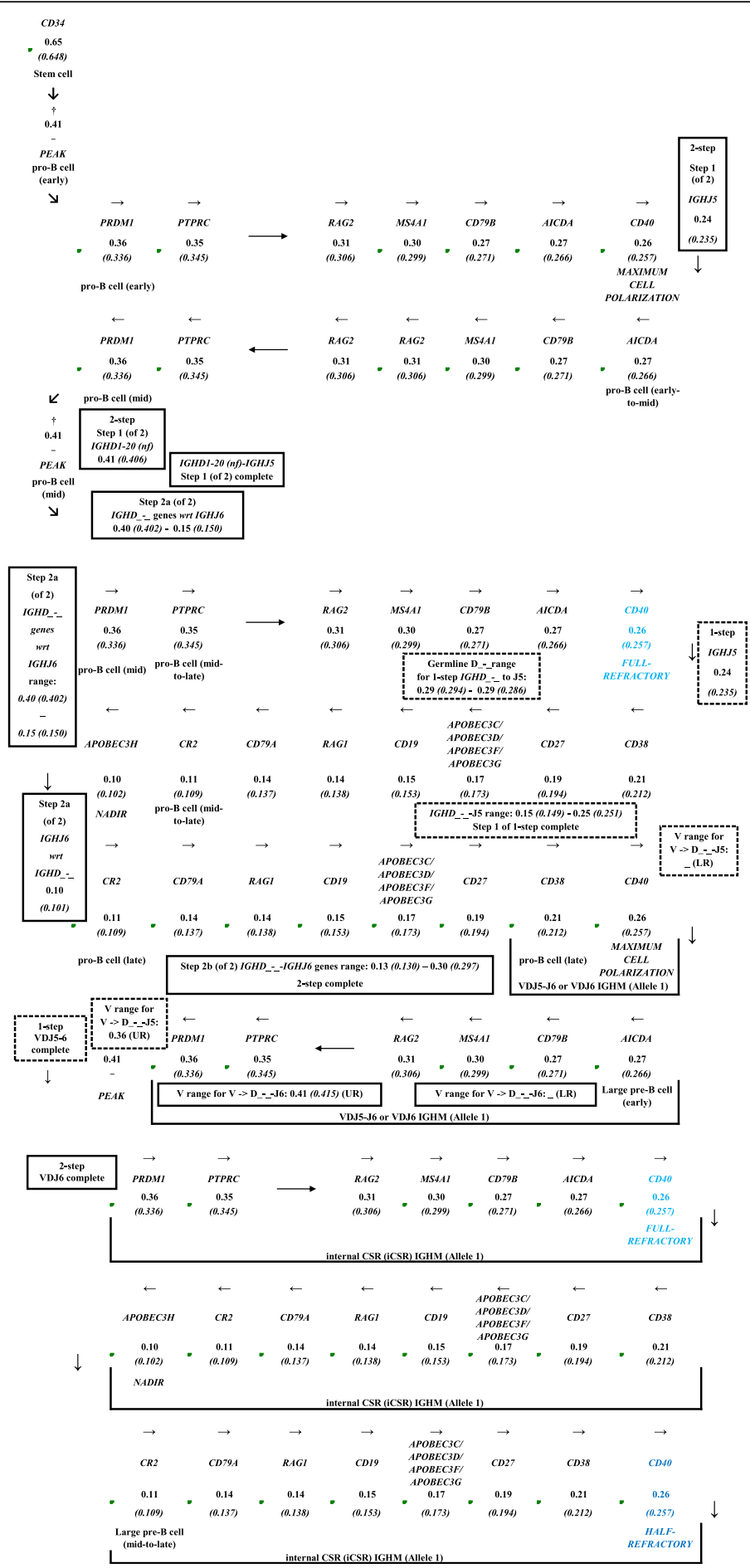

Fig. 1 Allele 1 (IGHM) 2-step and 1-step Ig heavy chain locus gene rearrangement recombination steps superimposed on the pressuromodulation map of B-cell differentiation stages. The Allele 1 two-step recombination steps are IGHD1-20 (nf) and IGHJ5 recombination (step 1 of 2), IGHD_-genes with respect to D1-20(nf)-J5 (step 2a of 2) and IGHJ6 with respect to D1-20(nf)-J5 (step 2a of 2) recombination that results that results in

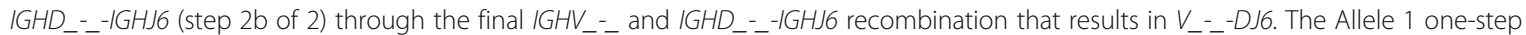
recombination step is IGHD__- and IGHJ5 recombination that results in IGHD_-_-IGHJ5 (step 1 of 1) through the final IGHV_-_ and IGHD_-_-J5 recombination that results in $V_{-}{ }_{-}-D J 6$. Note: Allele 1 locus rearrangement recombination steps complete to the point of the CM IgM+ B-cell before Allele 2 VDJ completes. †, upper esebssiwaago T $T_{Q}$ units range, 0.41-0.36. Black, CD40 at maximum cell polarization potential. Dark blue, CD40 at half-refractory. Light blue, CD40 at full-refractory. Text boxes with complete borders, 2-step. Text boxes with dashed borders, 1-step. Large rectangular box with complete borders, extra-nodal secretory antibody phase 


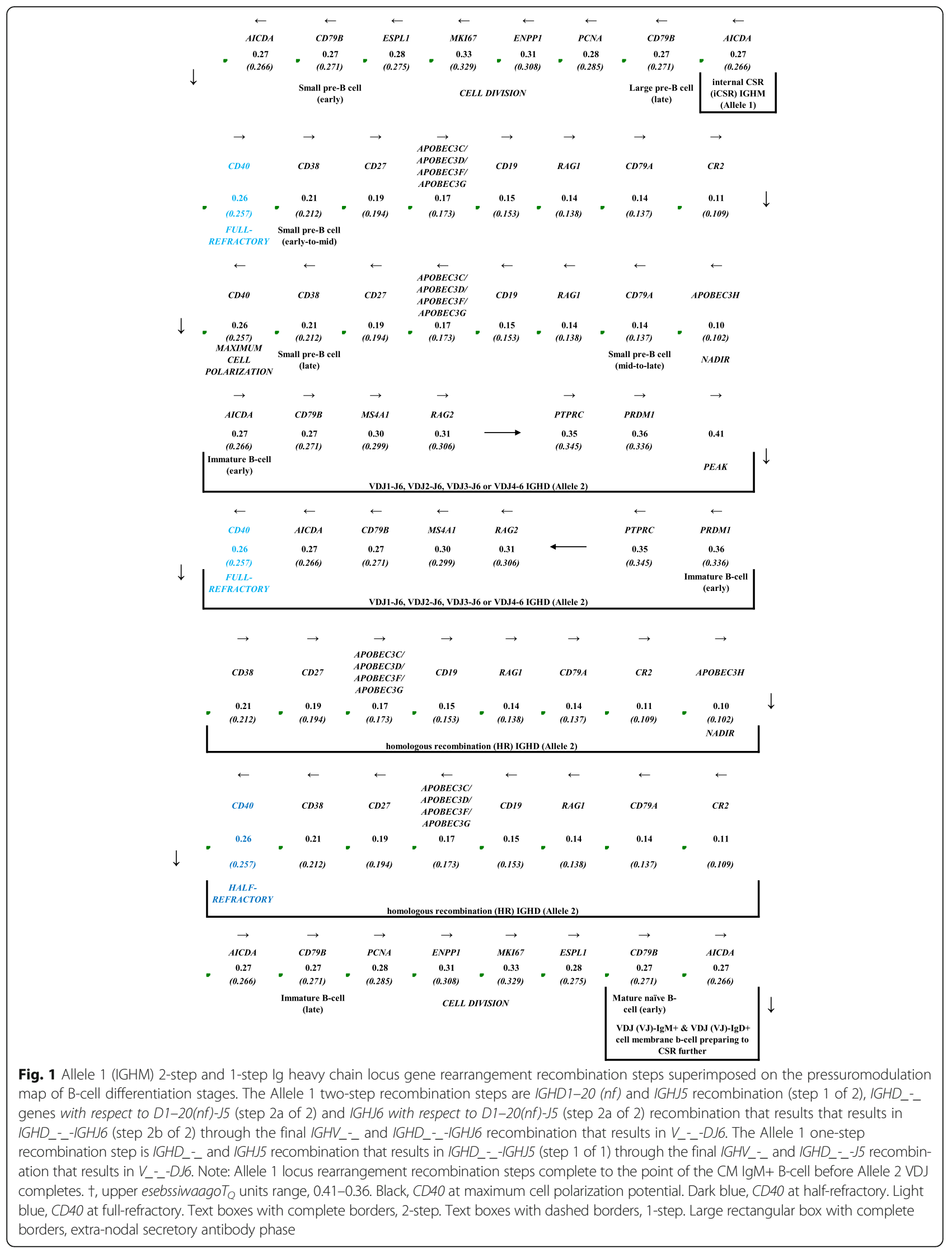




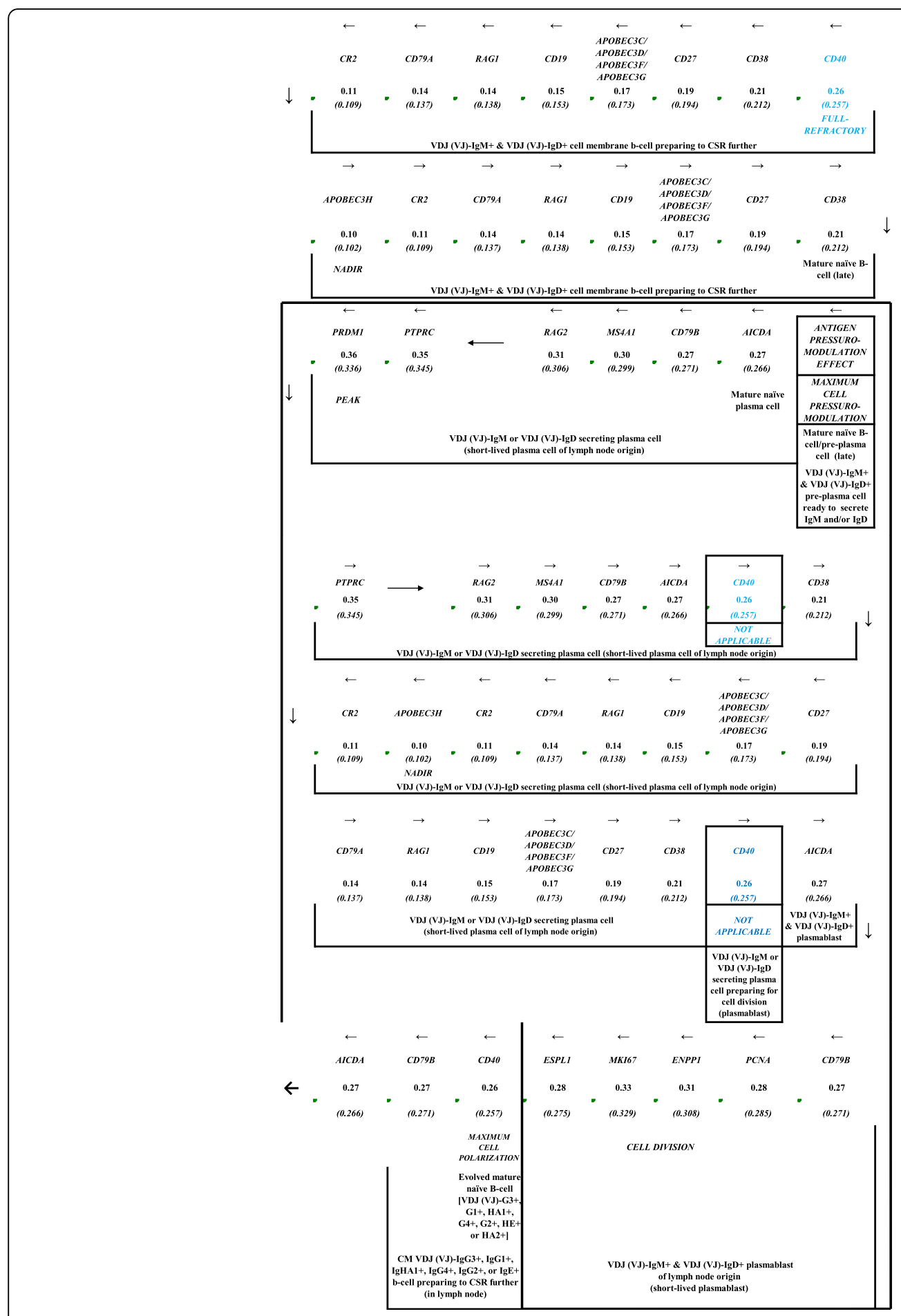

Fig. 1 Allele 1 (IGHM) 2-step and 1-step Ig heavy chain locus gene rearrangement recombination steps superimposed on the pressuromodulation map of B-cell differentiation stages. The Allele 1 two-step recombination steps are IGHD1-20 (nf) and IGHJ5 recombination (step 1 of 2), IGHD_-genes with respect to D1-20(nf)-J5 (step 2a of 2) and IGHJ6 with respect to D1-20(nf)-J5 (step 2a of 2) recombination that results that results in IGHD_-_-IGHJ6 (step 2b of 2) through the final IGHV_-_ and IGHD_-_-IGHJ6 recombination that results in $V_{-}$- $_{-} D J 6$. The Allele 1 one-step recombination step is /GHD_-_ and IGHJ5 recombination that results in IGHD_-_-IGHJ5 (step 1 of 1 ) through the final /GHV_-- and IGHD_-_J5 recombination that results in $V_{-}-_{-}-D J 6$. Note: Allele 1 locus rearrangement recombination steps complete to the point of the CM IgM+ B-cell before Allele 2 VDJ completes. $t$, upper esebssiwaago $T_{Q}$ units range, $0.41-0.36$. Black, CD40 at maximum cell polarization potential. Dark blue, CD40 at half-refractory. Light blue, CD40 at full-refractory. Text boxes with complete borders, 2-step. Text boxes with dashed borders, 1-step. Large rectangular box with complete borders, extra-nodal secretory antibody phase 


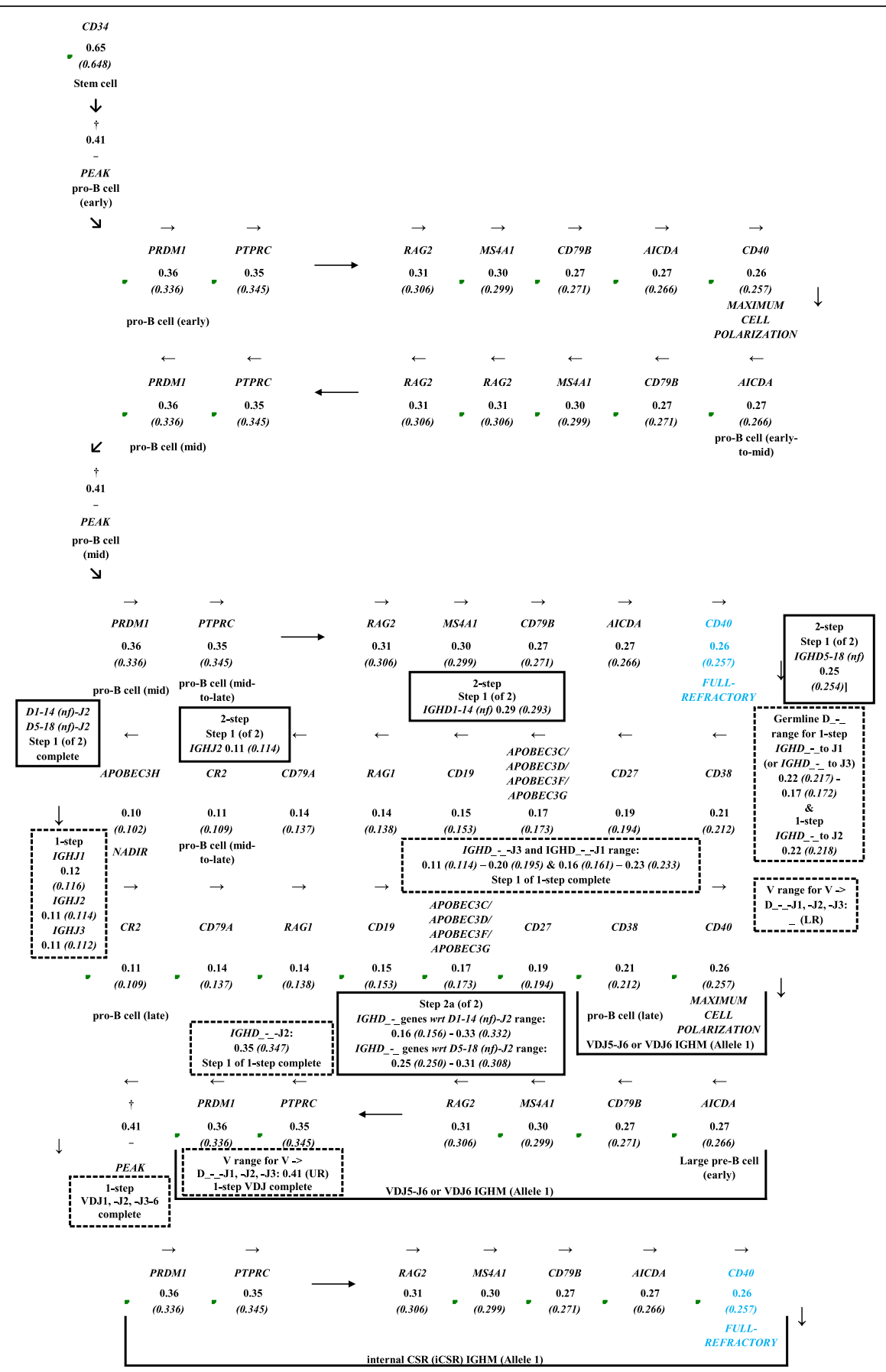

Fig. 2 Allele 2 (IGHD) 1-step and 2-step Ig heavy chain locus gene rearrangement recombination steps superimposed on the pressuromodulation map of B-cell differentiation stages. The Allele 2 one-step recombination step is IGHD_-_ and IGHJI, IGHJ2 or IGHJ3 recombination that results in IGHD_-_-IGHJ1 -J2 or -J3 (step 1 of 1) through the final IGHV_-- and IGHD_-_-IGHJ1, IGHD_-_-IGHJ2 or IGHD_-_-IGHJ3 recombination that results in $V_{-}--$DJ6. The Allele 2 two-step recombination steps are IGHD1-14 (nf) or IGHD5-18 (nf) and IGHJ2 recombination (step 1 of 2), the IGHD_-- genes with respect to D1-14 (nf)-J2 or D5-18 (nf)-J2 (step 2a of 2) and the IGHJ4 with respect to D1-14 (nf)-J2 or D5-18 (nf)-J2 (step 2a of 2) recombination that results in IGHD_-_-IGHJ4 (step 2b of 2) through the final IGHV_-_ and IGHD_-_-IGHJ4 recombination that results in $V_{-}$-_- $_{-}$JJ6. Note: Allele $2 \mathrm{lg}$ locus rearrangement recombination follows that of Allele 1 and always completes to the point of VDJ rearrangement in the marrow. In the classical pathway, homologous recombination or initial allelic exclusion followed by delayed Allele 2 iCSR (i.e. CM lgM+ IgD+) are the rule in the lymph node. In the non-classical pathway, Allele 2 locus recombination completes to the point of VDJ rearrangement (i.e CM IgM+ only). + , upper esebssiwaago $T_{Q}$ units range, $0.41-0.36$. Black, CD40 at maximum cell polarization potential. Dark blue, CD40 at half-refractory. Light blue, CD40 at full-refractory. Text boxes with complete borders, 2-step. Text boxes with dashed borders, 1-step. Large rectangular box with complete borders, extra-nodal secretory antibody phase 


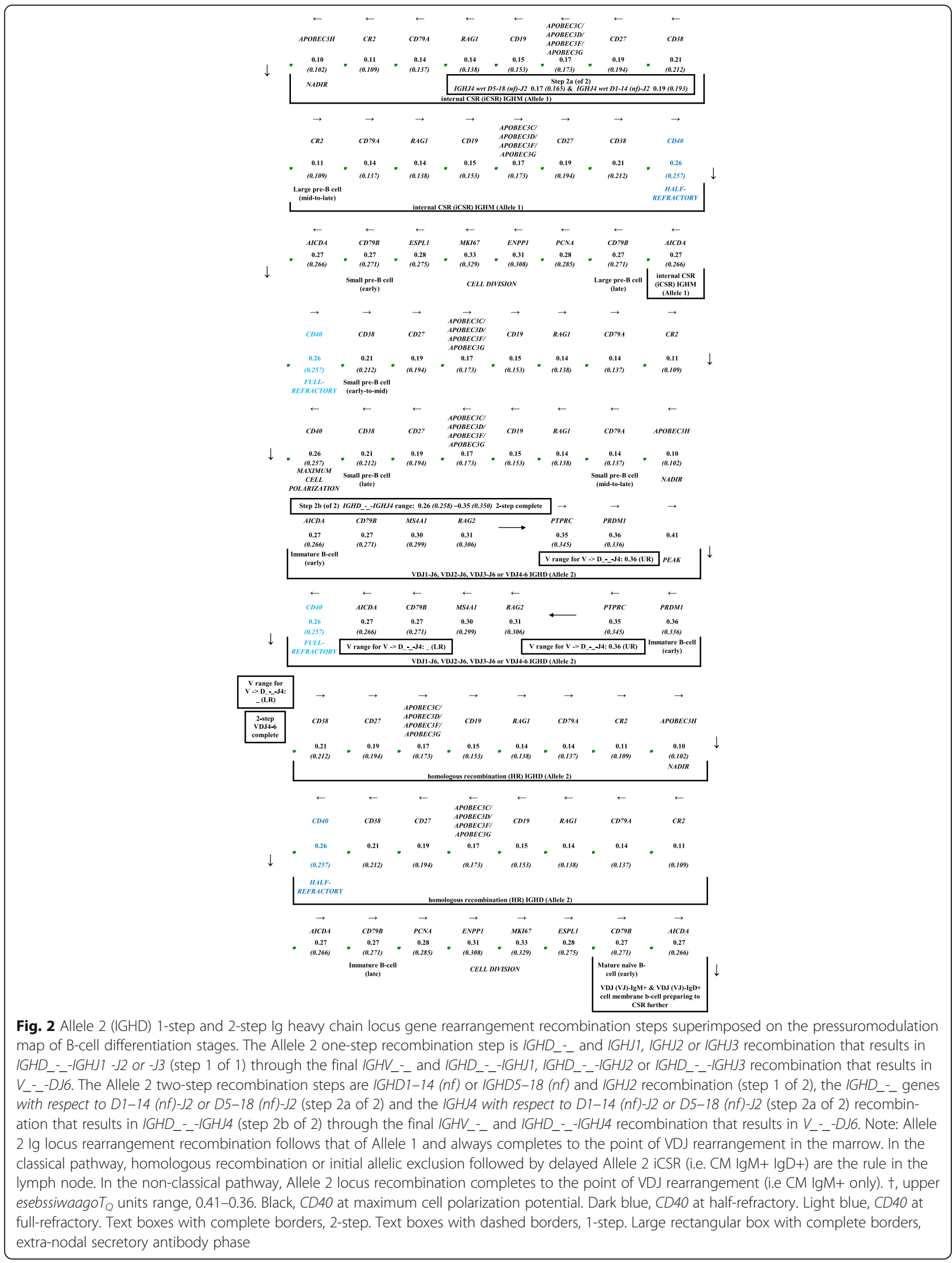




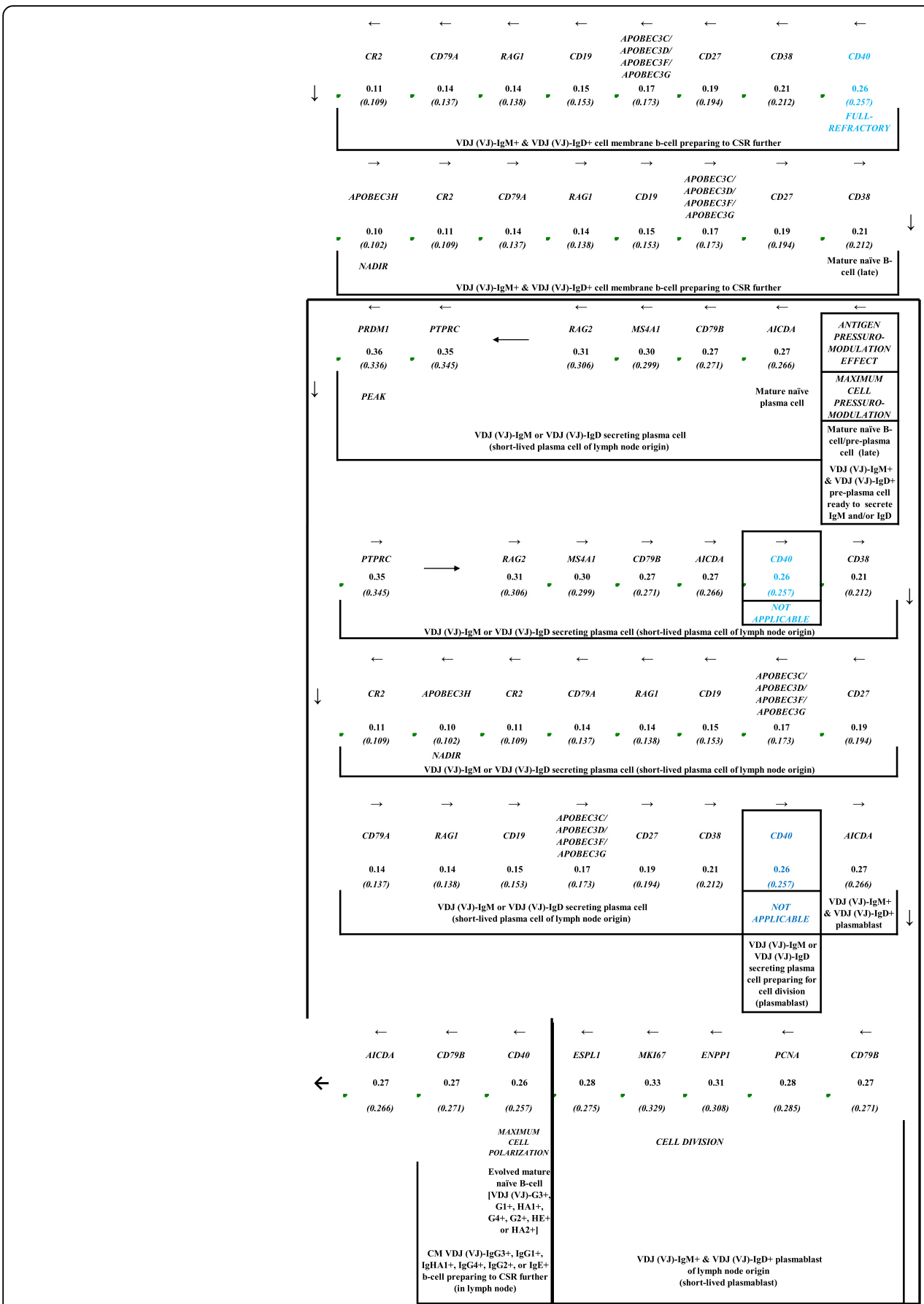

Fig. 2 Allele 2 (IGHD) 1-step and 2-step Ig heavy chain locus gene rearrangement recombination steps superimposed on the pressuromodulation map of B-cell differentiation stages. The Allele 2 one-step recombination step is IGHD_-_ and IGHJI, IGHJ2 or IGHJ3 recombination that results in IGHD_-_-IGHJ1 -J2 or -J3 (step 1 of 1) through the final IGHV_-- and IGHD_-_-IGHJ1, IGHD_-_-IGHJ2 or IGHD_-_-IGHJ3 recombination that results in $V_{-}--D J 6$. The Allele 2 two-step recombination steps are IGHD1-14 (nf) or IGHD5-18 (nf) and IGHJ2 recombination (step 1 of 2), the IGHD_-- genes with respect to D1-14 (nf)-J2 or D5-18 (nf)-J2 (step 2a of 2) and the IGHJ4 with respect to D1-14 (nf)-J2 or D5-18 (nf)-J2 (step 2a of 2) recombination that results in IGHD_-_-IGHJ4 (step 2b of 2) through the final IGHV_-_ and IGHD_-_-IGHJ4 recombination that results in V_-_-DJ6. Note: Allele $2 \mathrm{lg}$ locus rearrangement recombination follows that of Allele 1 and always completes to the point of VDJ rearrangement in the marrow. In the classical pathway, homologous recombination or initial allelic exclusion followed by delayed Allele 2 iCSR (i.e. CM lgM+ lgD+) are the rule in the lymph node. In the non-classical pathway, Allele 2 locus recombination completes to the point of VDJ rearrangement (i.e CM IgM+ only). + , upper esebssiwaago $T_{Q}$ units range, $0.41-0.36$. Black, CD40 at maximum cell polarization potential. Dark blue, CD40 at half-refractory. Light blue, CD40 at full-refractory. Text boxes with complete borders, 2-step. Text boxes with dashed borders, 1-step. Large rectangular box with complete borders, extra-nodal secretory antibody phase 
Allele 2 (IGHD) 2-step recombination involves germline IGHJ2 and IGHD4-11 (nf) or IGHD5-18 (nf) recombination (step 1), IGHD_-_ and IGHJ4 with respect to D411(nf)-J2 and D5-18(nf)-J2 gene recombination (step 2a) that results in IGHD_-_-IGHJ4 (step 2b) and then IGHV_-_ to IGHD_-_-IGHJ4 recombination that results in V_-_-DJ6

Step 1 of the Allele 2 two-step recombination process begins during the second downhill limb at an intracellular pressure of 0.29 esebssiwaago $T_{\mathrm{Q}}$ units with RAG association with either IGHD4-11 ( $n f$ ) (esebssiwaago $_{\mathrm{Q}}:$ 0.293) or IGHD5-18 (nf) (esebssiwaago $T_{\mathrm{Q}}$ : 0.254 ) followed by $I G H J 2$ association at 0.114 units during the $1^{\text {st }}$ peri-nadir, which results in IGHD4-11 (nf)-IGHJ2 or in IGHD5-18 (nf)-IGHJ2 (Fig. 2).

The step $2 \mathrm{a}$ of 2 of the process begins in the perinadir at $0.16(0.156)$ esebssiwaago $T_{Q}$ units and continues units into the uphill limb of the $2^{\text {nd }}$ threefourths maximum polarization period when intracellular pressure increases to 0.33 (0.332) units, which is the cell pressure range for $I G H D_{-}$- genes with respect to D1-14 (nf)-J2 and D5-18 (nf)-J2. Then, IGHD_-_ with respect to D1-14 (nf)-J2 or D5-18 (nf)-J2, and IGHJ4 with respect to D1-14 (nf)-J2 or IGHJ4 with respect to D5-18 ( $n f)-J 2$ gene recombination follows in the $3^{\text {rd }}$ three-fourths full-refractory period when the B-cell pressure is in the $0.19(0.193)$ to $0.17(0.165)$ esebssiwaago $T_{Q}$ units range, and ends in IGHD__-IGHJ4 when step $2 \mathrm{~b}$ is complete. There is no $I G H D_{-}$- to IGHJ4 gene recombination during the first $3 / 8^{\text {ths }}$ refractory period in between the $2^{\text {nd }}$ three-fourths maximum polarization period and the $3^{\text {rd }}$ three-fourths full-refractory period, when cell division takes place (Fig. 2).

The 2-step Allele 2 VDJ step begins with the 3rd threefourths maximum polarization period in the IGHD__-IGHJ4 recombined gene intracellular pressure range between 0.26 (0.258) and $0.35(0.350)$ esebssiwaago $T_{\mathrm{Q}}$ units, and completes in the $I G H V_{--}$gene with respect to IGHD_-_-IGHJ4 cell pressure range between an unestablished lower limit and the PRDM1 expression pressure of 0.36 units as the upper limit (Fig. 2) due to a less pressuromodulated B-cell.

Allele 2 recombination is a 2 -step process when $\mathrm{Al}$ lele 1 recombination is a 1 -step process as a less primed CD4R+ T-cell is involved, which results in lesser grade pressuromodulation of the B-cell. This implies that during a less robust pressuromodulation effect: (1) there is $34^{\text {ths }}$ of maximum polarization during the maximum polarization periods but sufficient enough for full PRDM1 expression at 0.36 esebssiwaa$g o T_{\mathrm{Q}}$ units, and (2) there is $34^{\text {ths }}$ of full refractoriness during the fully refractory periods during which there is only transient CD40 expression comparable to nonexpression.
Allele 1 (IGHM) 1-step recombination step involves germline IGHD_-_ and IGHJ5 recombination that results in IGHD_-_-IGHJ5 (step1) and then IGHV_-_ and IGHD_-_-J5 recombination that results in $V_{-}-{ }_{-} D J 6$

The Allele 1 one-step recombination process begins during the downhill limb after the 1st three-fourths maximal polarization period in between 0.29 (0.294) and $0.29(0.286)$ esebssiwaago $T_{\mathrm{Q}}$ units range when the genes destined for Allele 1 one-step are horizontal, and completes to the point of IGHD_-_-IGHJ5 at $0.24(0.235)$ esebssiwaago $T_{\mathrm{Q}}$ units just as the $1^{\text {st }}$ refractory period begins at which the 1-step IGHJ_ gene, IGHJ5 expresses (Fig. 1).

The step 1 IGHD_-_-IGHJ5 recombined gene B-cell pressure range is $0.15(0.149)$ to $0.25(0.251)$ and the $I G H V_{-}$-_ gene with respect to IGHD_-_-IGHJ5 esebssiwaago $_{\mathrm{Q}}$ range is the unestablished lower limit to 0.36 esebssiwaago $T_{\mathrm{Q}}$ units. The 1-step Allele $1 \mathrm{VDJ}$ begins in the peri-nadir and completes during the $2^{\text {nd }}$ threefourths maximum polarization period (Fig. 1).

The Allele 1 recombination process is a 1-step process during the less primed CD4R+ T-cell-mediated B-cell polarization effect. The Allele 1 one-step process can begin after the $1^{\text {st }}$ three-fourths maximal polarization period during the downhill limb as the intracellular pressure decreases at a slower rate compared to the Allele 2 two-step process into the three-fourths refractory CD4O expression period.

Variability gene esebssiwaago $T_{\mathrm{Q}} \mathrm{s}$ can be constant or variable The variability genes that were sampled from $5^{\prime}$ to $3^{\prime}$ include, IGHV4-61, IGHV4-59, IGHV5-51, IGHV3-48, $I G H V 4-28, I G H V 3-23$ and IGHV1-3. All of these except $I G H V 1-3$ have constant esebssiwaago $T_{\mathrm{Q}} \mathrm{s}$ with respect to germline $I G H D_{-}$-_ genes.

The esebssiwaago $T_{\mathrm{Q}}$ for $I G H V 1-3$ varies depending on the downstream location of IGHD_-_IGHJ6 in Allele 1 two-step or IGHD__-IGHJ4, IGHJ5 and IGHJ6 in series in Allele 2 two-step for example since the split integration includes the intergene distances downstream of these genes with respect to IGHVI-3.

\section{IGHV_-_ to IGHDJ_recombination results in VDJ6 for} both alleles

Variability gene to DJ_ gene recombination results in VDJ6 for both Allele 1 (IGHM) 1-step D_-_-J5 genes and for Allele 2 (IGHD) 1-step D_-_-J1, -J2, -J3 and 2step $D_{-}$-_-J4 genes.

After $I G H V_{-}$- $_{-}$to $I G H D_{-}$-_$_{-} I G H J 1$ for example, the $V_{-}$_-D__-J1 becomes a $V$ _-_-D___-J1-J6 gene as the VDJ promoters offer sufficient transcription factor-bound anchoring stability for RNA polymerase to be able to transcribe the entire V-to-J6 complex. The VDJ promoters however do not offer enough stability for transcription further 
downstream to immunoglobulin heavy chain M (IGHM), which requires internal consensus sequence recognition (iCSR) excision of intervening bases around the MIR genes for VDJ6-IGHM. Analogously, homologous recombination displacement removal of intervening bases results in VDJ6$I G H D$, which transcribes in toto as one gene.

This observation forms the basis for determining the gene esebssiwaago $T_{\mathrm{Q}}$ with respect to the correct VDJ, which is VDJ6.

\section{CD4R+ T-cells are positively pressuromodulated}

Antigen presenting cells (APC) scavenge endocytic antigens and re-present endocytic antigen fragments to the T-cell receptor (TCR) $[21,22]$ and other T-cell receptors [22]. Therefore, APC-mediated positive pressuromodulator antigen presentation to CD4R+ T-cells results in endocytic positive pressuromodulation of the CD4R+ T-cell.

More primed CD4R+ T-cells are subject to higher grades of positive pressuromodulation that results in maximal CD4OLG expression and CD40LG R+ T-cellmediated $\mathrm{CD} 40 \mathrm{R}+\mathrm{B}$-cell polarization effect that increases B-cell pressure to 0.41 esebssiwaago $T_{Q}$ units (Allele 1 two-step/Allele 2 one-step), while less primed T-cells are subject to lower grades of positive pressuromodulation, which only increases B-cell pressure into the PRDM1 expression range of 0.36 units (Allele 2 twostep/Allele 1 one-step).

Antigen presenting cell-dependent T-cell-mediated Bcell polarization is the primary mode of increasing B-cell pressure in the classical maturation pathway, where vaccines serve the purpose of boosting the response [23].

\section{B-cells are subject to the effect of both positive and negative forms of antigen pressuromodulation}

The effects of positive pressuromodulation out-weigh those of mixed or negative forms during B-cell maturation from the VDJ pro-B-cell stage to the consensus sequence recognition (CSR) isotype switching Evolved mature B-cell stage, as it is during oscillating positive pressuromodulation [4].

In the classical pathway the dominant form of pressuromodulation is T-cell-mediated B-cell polarization via CD40R, while in the non-classical pathway the dominant form is positive pressuromodulator antigen-mediated Bcell pressuromodulation such as toll-like receptor (TLR) [24]-mediated endocytic via high isoelectric point basic peptides such as profilin II [25] during acute infection for example.

The B-cell to plasma cell transformation takes place in the presence of an antigen load. The majority of antigens such as virus capsid peptide and microbe cell wall or membrane fragments [26] are positive pressuromodulators of cell surface receptors [1], which increase and maintain B-plasma cell pressure in the supra- pressuromodulated range ( $\geq 0.25$ esebssiwaago $T_{Q}$ units) [2]. A minority of antigens such as phospholipases and proteases [27] are negative pressuromodulators of cell membranes [1], which decrease and maintain B-plasma cell pressure in the infra-pressuromodulated range $(<0.25$ esebssiwaago $T_{Q}$ units) [2].

\section{IGHV1-3 antibody heavy chain recombination sequence after IGHV1-3-IGHD_-_-IGHJ6 is iCSR followed by further CSRs for allele 1 (IGHM) and homologous recombination for allele 2 (IGHD)}

For Allele 1 (IGHM), there is internal CSR (iCSR) [11] between the switch sequence region intergene bases of MIR4507 with respect to V1-3DJ6 (esebssiwaago $T_{Q}$ : 0.260 ) and the sequence intergene bases of MIR4539 with respect to V1-3DJ6 (esebssiwaago $T_{Q}: 0.268$ ), which is the closest esebssiwaago $T_{Q}$ match between the candidate $M I R_{-}$genes of the IGHM switch region. The resultant recombined gene is V1-3-D_-_J6-remaining MIR/ MIRs-IGHM with an esebssiwaago $T_{Q}$ of 0.275 (Table 7), and the cell a CM IgM+ Mature naïve B-cell.

For Allele 2 (IGHD), there is homologous recombination displacement replacement of 442 intergene bases downstream of IGHJ6 of V1-3-D_-_-IGHJ6 (esebssiwaagoT $\left.T_{Q}: 0.226\right)$ by 443 intergene bases upstream of $I G H D$ with respect to V1-3-D_-_-IGHJ6 (esebssiwaago $T_{Q}$ : 0.198) [14] due to an esebssiwaago $T_{Q}$ match between the two genes, at minus 0.014 units for the former (IGHD) and plus 0.014 units for the latter $(V 1-2-D J 6)$, which meets the esebssiwaago $T_{Q}$ match criterion of \pm 0.015 units with reference to the gene as it is the point of convergence. The resultant gene is V1-3D_-_J6-IGHD with an esebssiwaago $_{Q}$ of 0.320 (Table 7).

For Allele 1 (IGHM), the downstream genes for further CSR to V1-3DJ6-IGHM (esebssiwaago $T_{Q}:$ 0.275) are IGHG3 (esebssiwaagoT $T_{Q}: 0.271$ ) and IGHA2 (esebssiwaa$\left.g o T_{Q}: 0.258\right)$, but not IGHG1, IGHA1, IGHG4/IGHG2 and IGHE. The esebssiwaago $T_{Q}$ match for IGHG3 with respect to $V 1-3 D J-I G H M$ is plus minus 0.002 units and that for IGHA2 is plus minus 0.0085 units. The former will be a primary IGHG3 switch-to-IGHM switch region CSR resulting in V1-3DJ6-IGHG3 with an esebssiwaago $T_{Q}$ of 0.306 , and the latter will be a direct primary IGHA2 switch-to-IGHM switch region CSR resulting in V1-3DJ6IGHA2 with an esebssiwaago $T_{Q}$ of 0.258 (Table 7).

After primary CSR, the CD4R+ T-cell polarized $1^{\text {st }}$ generation Evolved mature naïve nodal B-cell will express both V1-3DJ6-IGHG3 (esebssiwaago $T_{Q}$ : 0.306) and V1-3DJ6-IGHD (esebssiwaago $T_{Q}:$ 0.320) simultaneously when the B-cell intracellular pressure oscillates to 0.313 esebssiwaago $T_{Q}$ units as V1-3DJ6-IGHG3 (+ $0.007)$ and V1-3DJ6-IGHD $(-0.007)$ are within 0.014 units of each other. Likewise, an antigen stimulated extra-nodal B-plasma cell will secrete both IgG3 
and IgD (see Fig. 1 or Fig. 2 periphery/tissue nidus secretory phase).

The only secondary CSR for a CM IgG3+/IgD+ $1^{\text {st }}$ generation Evolved mature naïve nodal B-cell is IGHG4 with respect to V1-3DJ6-IGHG3 (esebssiwaago $T_{Q}$ : 0.325) at plus minus 0.0095 units, which will result in V13DJ6-IGHG4 with an esebssiwaagoT $T_{Q}$ of 0.237 (Table 7).

The further CSR for the CM IgG4+ $2^{\text {nd }}$ generation nodal cell will be a tertiary CSR to IGHA2 with respect to V1-3DJ6-IGHG4 (esebssiwaago $T_{Q}$ of 0.226), where there is a match at plus minus 0.0055 units, which results in $\mathrm{CM}$ IgHA $+3^{\text {rd }}$ generation nodal cell. After both direct primary CSR between IGHA2 and V1-3DJ6-IGHM and indirect tertiary CSR between IGHA2 and V1-3DJ6IGHG4, the resultant gene is the same, V1-3DJ6-IGHA2 expressed at 0.185 units, one expressed by a $1^{\text {st }}$ generation cell and the other by a $3^{\text {rd }}$ generation cell.

\section{IGHV3-23 antibody heavy chain recombination sequence} after IGHV3-23-IGHD_-_-IGHJ_-J6 is iCSR followed by further CSRs for allele 1 (IGHM) and initial allelic exclusion for allele 2 (IGHD)

For Allele 1 (IGHM), there is internal CSR between the sequence intergene bases of MIR4537 with respect to V3-23DJ6 (esebssiwaago $T_{Q}:$ 0.272) and the intergene bases of MIR4539 with respect to V3-23DJ6 (esebssiwaa$\left.g o T_{Q}: 0.277\right)$, which is the closest esebssiwaago $T_{Q}$ match and results in V3-23-D_-_J6-remaining MIR/MIRsIGHM with an esebssiwaago $T_{Q}$ of 0.277 (Table 8).

For Allele 2 (IGHD), homologous recombination does not take place between IGHD (esebssiwaago $T_{Q}: 0.208$ ) and V3-23-D_-_-J6 (esebssiwaago $T_{Q}: 0.285$ ) as there is no esebssiwaago $T_{Q}$ match (Table 8). Instead, delayed internal CSR will follow, which will result in Allele $2 \mathrm{V3}-$ 23-D_-_J6-remaining MIR/MIRs-IGHM and in further CSRs analogous to Allele 1.

For both alleles, the primary CSR esebssiwaago $T_{Q}$ match genes for V3-23DJ6-IGHM (esebssiwaago $T_{Q}$ : 0.277 ) are IGHG1 (esebssiwaagoT $T_{\mathrm{Q}}: 0.271$ ) and IGHA2 (esebssiwaago $T_{\mathrm{Q}}: 0.258$ ), respectively. A primary direct IGHG1-to-IGHM CSR at plus minus 0.003 units results in V3-23DJ6-IGHG1 that expresses at an esebssiwaa$g o T_{Q}$ of 0.256 , and a primary direct IGHA2-to-IGHM CSR at plus minus 0.0095 units results in V3-23DJ6IGHA2 that expresses at an esebssiwaago $T_{Q}$ of 0.208 (Table 8).

The CM IgG1+/IgG1+ $1^{\text {st }}$ generation Evolved mature naïve B-cell secondary CSRs with IGHA1 with respect to V3-23DJ6-IGHG1 with an esebssiwaago $T_{Q}$ of 0.263 that is sufficiently horizontal at minus 0.0035 units in reference to V3-23DJ6-IGHG1, which is the only potential CSR for V3-23DJ6-IGHG1 with a downstream gene and results in V3-23DJ6-IGHA1 with an esebssiwaago $T_{Q}$ of 0.171 (Table 8).
For the population of isotype switching B-cells, there are two potential tertiary CSRs for a CM IgA1+/IgA1+ $2^{\text {nd }}$ generation-Evolved mature naïve $B$-cell with an esebssiwaago $T_{Q}$ of 0.171 units, one with IGHE with respect to V3-23DJ6-IGHA1 at 0.167 units $( \pm 0.002)$, and the other with IGHA2 with respect to V3-23DJ6-IGHA1 at 0.179 units $( \pm 0.004)$. The further quaternary CSR for $\mathrm{CM} \operatorname{IgE}+/ \mathrm{IgE}+3^{\text {rd }}$ generation-Evolved mature naïve $\mathrm{B}$ cell at 0.205 esebssiwaago $T_{Q}$ units is with IGHA2 with respect to V3-23DJ6-IGHE at an esebssiwaago $T_{Q}$ of 0.183 units $( \pm 0.011)$ (Table 8).

The higher generation-Evolved mature naïve cells in reference to V3-23DJ6-IGHM are more somatically mutated B-cells to the point of B-cell to B-plasma cell transformation as they are the product of multiple CSRs [28], particularly when CSRing around the intracellular pressures at which the somatic hypermutation (SHM) cytidine deaminases (CDA) are maximally expressed and available [4].

IGHV5-51 antibody heavy chain recombination sequence after IGHV5-51-IGHD_-_-IGHJ_-J6 is iCSR followed by further CSRs for allele 1 (IGHM) and initial allelic exclusion for allele 2 (IGHD)

For Allele 1 (IGHM), there is internal CSR between the sequence intergene bases of MIR4537 with respect to V5-51DJ6 (esebssiwaagoT $T_{Q}$ : 0.152) and those of MIR4507/MIR4538 with respect to V5-51DJ6 (esebssiwaago $_{Q}:$ 0.155), which is the closest esebssiwaago $T_{Q}$ match and results in V5-51-D_-_-J6-remaining MIR/ MIRs-IGHM with an esebssiwaago $T_{Q}$ of 0.165 (Table 9).

For Allele 2 (IGHD), homologous recombination does not take place between IGHD (esebssiwaago $T_{Q}: 0.169$ ) and V5-51-D_-_-J6 (esebssiwaago $T_{Q}:$ 0.233), internal CSR takes place on both alleles (Table 9).

There are four potential primary CSR esebssiwaago $T_{Q}$ match genes for V5-51DJ6-IGHM (esebssiwaago $T_{Q}$ : 0.165), IGHG1 that is horizontal at 0.160 units $( \pm 0.0025), I G H A 1$ that is horizontal at 0.180 units $( \pm 0.0075)$, IGHG4 that is horizontal at 0.189 units $( \pm 0.012)$, and IGHA2 that is horizontal at 0.175 units $( \pm 0.005)$, where the choice of further CSR depends on the B-cell pressure as it oscillates with cyclic periodicity in context of the local milieu. Primary CSR with V551DJ6-IGHM will result in either V5-51DJ6-IGHG1 (esebssiwaago $T_{Q}$ : 0.153), V5-51DJ6-IGHA1 (esebssiwaagoT $_{Q}$ : 0.134), V5-51DJ6-IGHG4 (esebssiwaago $T_{Q}: 0.184$ ) or V5-51DJ6-IGHA2 (esebssiwaago $T_{Q}: 0.148$ ), and in a $1^{\text {st }}$ generation evolved B-cell (Table 9).

There are also four potential downstream secondary CSR esebssiwaago $T_{Q}$ match genes for V5-51DJ6-IGHG1 (esebssiwaago $T_{Q}$ : 0.153$), I G H A 1$ at 0.166 units $( \pm 0.0065)$, IGHG4 at 0.137 units $( \pm 0.008), I G H E$ at 0.145 units $( \pm 0.004)$, and $I G H A 2$ at 0.157 units $( \pm 0.002)$. Secondary 
CSR with V5-51DJ6-IGHG1 will result in the recombined genes as before expressing at the their respective esebssiwaago $T_{Q} \mathrm{~s}$, in either V5-51DJ6-IGHA1, V5-51DJ6IGHG4, or V5-51DJ6-IGHA2 and in addition, V551DJ6-IGHE that expresses at 0.152 units, and in a $2^{\text {nd }}$ generation evolved B-cell (Table 9).

There are three potential downstream tertiary CSR esebssiwaago $T_{Q}$ match genes for V5-51DJ6-IGHA1 (esebssiwaago $T_{Q}$ : 0.134), IGHG4 at 0.106 units $( \pm 0.014)$, IGHE at 0.153 units $( \pm 0.0095)$, and IGHA2 at 0.163 units $( \pm 0.00145)$. Tertiary CSR with V5-51DJ6-IGHA1 will result in the recombined genes as after the prior isotype switch, in either V5-51DJ6-IGHG4, V5-51DJ6-IGHE or V5-51DJ6-IGHA2, and in a $3^{\text {rd }}$ generation evolved B-cell (Table 9). Thus, tertiary CSRed $3^{\text {rd }}$ generation evolved B-cell plasma cells will express and secrete antibody that is somatically hypermutated, for example IgE that is the product of three sequential CSRs (V5-51DJ6-IGHM $\longrightarrow$ V5-51DJ6-IGHA1 $\longrightarrow$ V5-51DJ6-IGHG1 $\longrightarrow$ V5-51DJ6IGHE) [29].

There is no potential for a further quaternary CSR from V5-51DJ6-IGHG4 (esebssiwaago $T_{Q}$ : 0.184), as there is no match with downstream genes, IGHE has an esebssiwaago $T_{Q}$ of 0.146 , while IGHA2 has an esebssiwaago $T_{Q}$ of 0.153 , both being outside of the match range, IGHE at plus minus 0.019 units and IGHA2 at plus minus 0.0155 units when neither DNA segment is horizontal enough for stable CSR by cytidine deaminases [4] (Table 9).

There is the potential for a quaternary CSR between IGHA2 with respect to V5-51DJ6-IGHE (esebssiwaagoT $T_{\mathrm{Q}}$ : 0.129) and upstream V5-51DJ6-IGHE (esebssiwaa$\left.g o T_{\mathrm{Q}}: 0.152\right)$ as its plus minus 0.0115 units at the point of gene esebssiwaago $T_{\mathrm{Q}}$ convergence (Table 9),

During the secretory phase, B-plasma cells with recombined V5-51 antibody heavy chain genes will express the V5-51DJ6-IGH antibody gene in response to negative pressuromodulation antigens of the cell membrane such as lipases and proteases.

\section{Clinical correlation with allergen-induced immunogenicity} Allergic disease states such as rhinitis, asthma and venom sensitivity are subsets of IgE-mediated Type I hypersensitivity, the former two caused by mucosal exposure to plant pollen (i.e. Amb a series) and arthropod excreta $(\operatorname{Der} p)$ for example $[27,30]$, and the latter one caused by intravascular exposure to hymenoptera venoms (bee, Api m; jacket, Ves g; wasp, Pol a) [31].

Pollen and mite excreta are contaminated with endotoxins such as bacterial lipopolysaccharide (LPS) [32] and fungal profilin II $[24,25]$ and proteases [33]; while, honey bee venom apitoxin contains gland melittin (Api $m$ III) [34], mast cell degranulating peptide 401 [35] and enzymes hyaluronidase (Api $m$ II), phospholipase A2 (Api $m$ 1), acid phosphatase (Api $m$ IV) and dipeptidyl peptidase 4 (Api $m \mathrm{~V})[31,36,37]$.

In the classical pathway, antigen presenting cells (APC) scavenge and endocytose agglomerated nanoparticulates such as pollen-coated with lipopolysaccharide [38, 39] and melittin peptide lysed-cell membrane complexes [34] and re-present previously encountered endocytic positive pressuromodulator antigen fragments to CD4R+ T-cells for subsequent $\mathrm{T}$-cell-mediated B-cell polarization pressuromodulation; while, in the non-classical pathway, dissoluted monomeric forms of LPS and profilin II positively pressuromodulate B-cells via endocytosis [40], as does melittin [41] however in concentrated form it is cell lytic as it is a very basic peptide [42].

In atopic allergic disease, antigen-specific serum IgE antibody is present; it is a product of multiple sequential indirect CSRs at the intracellular pressures at which there is maximal expression of somatic hypermutation (SHM) enzymes [4], as is the case in atopic rhinitis [20, 43], asthma [44, 45] and venom sensitivity [46, 47].

In bee venom sensitization and asthma for example, over time there is a shift from somatically hypermutated specific IgE to specific IgG4 [45-47]. The IgG4 is less mutated than IgE which CSR equivalently, probably since the PRDM1-induced drop in B-cell pressure to between 0.10 and 0.12 units is transient during the perinadir when SHM enzyme APOBEC3H is expressed [4]. The basis for the shift from IgE to IgG4 is probably a shift in B-cell pressure from a higher pressure at around 0.144 esebssiwaago $T_{\mathrm{Q}}$ units required for CSR to IGHE to a lower pressure at around 0.124 esebssiwaago $T_{\mathrm{Q}}$ units required for CSR to IGHG4 (i.e. V5-51), due to the exposure of local lymph node B-cells to similar concentrations of negative antigen pressuromodulator enzymes such as phospholipase A2 during each successive sting, but to decreasing concentrations of positive antigen pressuromodulators (i.e. LPS) due to increasing endocytic efficiency of scavenging cells.

\section{Conclusions}

In this study, the recently developed gene esebssiwaago $T_{\mathrm{Q}}$-based $\mathrm{B}$-cell maturation stage gene overexpression pressuromodulation map [4] has been utilized as a template to stimulate B-cell immunoglobulin locus recombination events that take place in the pressuromodulated state in vivo. Germline joining-to-diversity gene rearrangements have been performed with respect to the germline followed by variability-to-diversityjoining gene recombinations through further consensus sequence recognition (CSR) isotype switching recombinations with respect to their recombined position.

Based on the findings of this study the following inferences can be made: (1) the esebssiwaago $T_{\mathrm{Q}}$ of a joining 
$\left(U_{-}\right)$and diversity $\left(D_{-}-\right)^{-}$gene in its native germline configuration is the basis for predictable subsequent gene rearrangement; (2) $D_{-}-$to $J_{-}$gene recombination events are bi-allelic and mutually exclusive; (3) the entire process from beginning to end depends on the grade of the pressuromodulation effect, and as per the classical pathway it is an antigen presenting cell (APC)dependent $\mathrm{CD} 4 \mathrm{R}+\mathrm{T}$-cell-mediated B-cell polarization process; (4) CD4R+ T-cells are positively pressuromodulated, while B-cells are subject to the effect of both positive and negative forms of antigen pressuromodulation; and (5) the B-cell to plasma cell transformation and the extra-nodal periphery/tissue nidus phase take place in the presence of antigen load and either positive or negative pressuromodulation of the cell to its recombined antibody gene expression intracellular pressure.

B-cell gene recombination rearrangement events can be predicted with a reasonable degree of certainty. It is envisioned that further esebssiwaago $T_{\mathrm{Q}}$-based study of the remaining B-cell variability gene recombinations isotype switching events will further our understanding of pressuromodulated basis for antigen selection including the evolutionary underpinnings of.

\section{Additional files}

Additional file 1: Table S1. Chromosome 14 lg heavy chain locus mined location data. (PDF $375 \mathrm{~kb}$ )

Additional file 2: Table S2. Chromosome 14 (-) strand chromatin lg heavy chain locus immunoglobulin gene esebssiwaago $T_{Q} S$ to final 2-digit (and 3-digit) esebssiwaago $T_{Q}$ for germline genes in native $5^{\prime}->3^{\prime}$ chronology before gene rearrangement ${ }^{1(\mathrm{a})}$ Episodes beginning with an anisotropic (A) or mesotropic (M) SEB; ${ }^{1(b)}$ non-contributory anisotropic subepisode block (NCA); ${ }^{1(c)}$ non-contributory single or multiple stabilizing isotropy points or reverse stabilizing isotropy point(s), NCstl; ${ }^{1(d)}$ anisotropy converted-to-mesotropy, ACM; and ${ }^{1(\mathrm{e})}$ indirect reverse stlsotropy and/or stlsotropy for anisotropy or for mesotropy, stMfA or stMfM. (DOC 51 kb)

Additional file 3: Table S3. Chromosome $14(-)$ strand chromatin lg heavy chain locus joining gene esebssiwaago $T_{Q} S$ to final 2-digit (and 3-digit) esebssiwaago $T_{Q}$ for germline genes in native $5^{\prime}->3^{\prime}$ chronology before gene rearrangement ${ }^{1(a)}$ Episodes beginning with an anisotropic (A) or mesotropic (M) SEB; ${ }^{1 \text { (b) }}$ non-contributory anisotropic sub-episode block (NCA); ${ }^{1(\mathrm{C})}$ non-contributory single or multiple stabilizing isotropy points or reverse stabilizing isotropy point(s), NCstl; ${ }^{1(d)}$ anisotropy converted-to-mesotropy, ACM; and ${ }^{1(\mathrm{e})}$ indirect reverse stlsotropy and/or stlsotropy for anisotropy or for mesotropy, stMfA or stMfM. (DOC $44 \mathrm{~kb}$ )

Additional file 4: Table S4. Chromosome 14 (-) strand chromatin lg heavy chain locus diversity gene esebssiwaago $T_{Q} S$ to final 2-digit (and 3-digit) esebssiwaago $T_{Q}$ for germline genes in native $5^{\prime}$ - > 3' chronology before gene rearrangement ${ }^{1(a)}$ Episodes beginning with an anisotropic (A) or mesotropic (M) SEB; ${ }^{1(b)}$ non-contributory anisotropic sub-episode block $(\mathrm{NCA}){ }^{1(\mathrm{c})}$ non-contributory single or multiple stabilizing isotropy points or reverse stabilizing isotropy point(s), NCstl; ${ }^{1(\mathrm{~d})}$ anisotropy converted-tomesotropy, ACM; and ${ }^{1(\mathrm{e})}$ indirect reverse stlsotropy and/or stlsotropy for anisotropy or for mesotropy, stMfA or stMfM. ${ }^{*}$ For IGHD1-20 nf , NCA of SEB no. 7 due to reverse anisotropy preceding original ending confirmation mesotropic SEB (no. 8) which sums into SEB no. 6 with inclusion of anisotropic SEB no. 9 as the ending SEB, which precedes the new ending confirmation mesotropic SEB (no. 10) (final SEB count is $7^{*}$ ) ${ }^{*}$ For IGHD1-1, ACM of initial anisotropic ending confirmation SEB no. 8 due to 0.25 -factor-adjusted reverse stlsotropy preceding SEB (no. 8), which sums into initial mesotropic
SEB no. 7, and the new ending confirmation anisotropic SEB is no. 9 (final SEB count is $\left.7^{*}\right)$. (DOC $71 \mathrm{~kb}$ )

Additional file 5: Table S5. Chromosome $14(-)$ strand chromatin lg heavy chain locus diversity (D)-to-joining $(J)$ recombination sequence esebssiwaago $T_{Q}$ s to final 2-digit (and 3-digit) esebssiwaago $T_{\mathrm{Q}}$ before VDJ for allele $1(\mathrm{IGHM})^{1(\mathrm{a})}$ Episodes beginning with an anisotropic (A) or mesotropic (M) SEB; ${ }^{1(\mathrm{~b})}$ non-contributory anisotropic sub-episode block (NCA); ${ }^{1(c)}$ noncontributory single or multiple stabilizing isotropy points or reverse stabilizing isotropy point(s), NCstl; ${ }^{1(\mathrm{~d})}$ anisotropy converted-to-mesotropy, ACM; and ${ }^{1(\mathrm{e})}$ indirect reverse stlsotropy and/or stlsotropy for anisotropy or for mesotropy, stMfA or stMfM. (DOC 66 kb)

Additional file 6: Table S6. Chromosome 14 (-) strand chromatin lg heavy chain locus diversity (D)-to-joining $(J)$ recombination sequence esebssiwaago $T_{Q} S$ to final 2-digit (and 3-digit) esebssiwaagoto before VDJ for allele $2(\mathrm{IGHD})^{1(\mathrm{a})}$ Episodes beginning with an anisotropic (A) or mesotropic (M) SEB; ${ }^{1(\mathrm{~b})}$ noncontributory anisotropic sub-episode block (NCA); ${ }^{1(c)}$ non-contributory single or multiple stabilizing isotropy points or reverse stabilizing isotropy point(s), $\mathrm{NCstl}$; ${ }^{1(\mathrm{~d})}$ anisotropy converted-to-mesotropy, ACM; and ${ }^{1(\mathrm{e})}$ indirect reverse stlsotropy and/or stlsotropy for anisotropy or for mesotropy, stMfA or stMfM. (DOC 76 kb)

Additional file 7: Table S7. Chromosome 14 (-) strand chromatin lg heavy chain locus variability gene esebssiwaago $T_{Q} S$ to final 2-digit (and 3-digit) esebssiwaago $T_{Q}$ for germline genes in native $5^{\prime}->3^{\prime}$ chronology ${ }^{1(a)}$ Episodes beginning with an anisotropic (A) or mesotropic (M) SEB; ${ }^{1(b)}$ non-contributory anisotropic sub-episode block (NCA); ${ }^{1(c)}$ noncontributory single or multiple stabilizing isotropy points or reverse stabilizing isotropy point(s), NCstl; ${ }^{1(d)}$ anisotropy converted-to-mesotropy, $\mathrm{ACM}$; and ${ }^{1(\mathrm{e})}$ indirect reverse stlsotropy and/or stlsotropy for anisotropy or for mesotropy, stMfA or stMfM. ${ }^{\S} / n c-A L 901608.1-10 /$ IGHV1-3/IGHV4-4

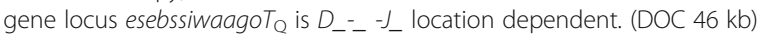

Additional file 8: Table S8. Chromosome 14 (-) strand chromatin Ig heavy chain locus gene recombination sequence esebssiwaago $T_{Q} S$ to final 2-digit (and 3-digit) esebssiwaago T for both alleles after IGHV1-3-IGHD_$-I G H J 6^{1(\mathrm{a})}$ Episodes beginning with an anisotropic (A) or mesotropic (M) SEB; ${ }^{1(b)}$ non-contributory anisotropic sub-episode block (NCA); ${ }^{1(c)}$ noncontributory single or multiple stabilizing isotropy points or reverse stabilizing isotropy point(s), NCstl; ${ }^{1(\mathrm{~d})}$ anisotropy converted-to-mesotropy, $\mathrm{ACM}$; and ${ }^{1(\mathrm{e})}$ indirect reverse stlsotropy and/or stlsotropy for anisotropy or for mesotropy, stMfA or stMfM. For IGHV1-3-IGHD_-_-IGHJ6, *ACM within initial anisotropic SEB no. 4, which results in a final SEB count of 9 $[7(+2): 9]$, and then stIMfA of initial anisotropic ending confirmation SEB no. 8 (final SEB no. 10) due to 0.25 -factor-adjusted reverse stlsotropy preceding SEB (no. 10), which sums into initial mesotropic SEB no. 9, and the new ending confirmation anisotropic SEB is no. 11 (final SEB count is $9^{*}$ ). For V1-3-D_-_-J_-J6-IGHG4, *ACM of initial anisotropic ending confirmation SEB no. 5 due to 0.25-factor-adjusted reverse stlsotropy preceding SEB (no. 5), which sums into $f$ mesotropic SEB no. 4, and the new ending confirmation anisotropic SEB is no. 7 (final SEB count is $5^{*}$ ). (DOC $74 \mathrm{~kb}$ )

Additional file 9: Table S9. Chromosome 14 (-) strand chromatin lg heavy chain locus gene recombination sequence esebssiwaago $T_{\mathrm{Q}} \mathrm{S}$ to final 2-digit (and 3-digit) esebssiwaago $T_{\mathrm{Q}}$ for both alleles after IGHV3-23IGHD_-_IGHJ6 ${ }^{1(\mathrm{a})}$ Episodes beginning with an anisotropic (A) or mesotropic (M) SEB; ${ }^{1(\mathrm{~b})}$ non-contributory anisotropic sub-episode block $(\mathrm{NCA}){ }^{1(\mathrm{c})}$ non-contributory single or multiple stabilizing isotropy points or reverse stabilizing isotropy point(s), NCstl; ${ }^{1(\mathrm{~d})}$ anisotropy converted-tomesotropy, ACM; and ${ }^{1(\mathrm{e})}$ indirect reverse stlsotropy and/or stlsotropy for anisotropy or for mesotropy, stMfA or stMfM. (DOC 74 kb)

Additional file 10: Table S10. Chromosome 14 (-) strand chromatin lg heavy chain locus gene recombination sequence esebssiwaago $T_{Q} S$ to final 2digit (and 3-digit) esebssiwaago T for both alleles after IGHV5-51-IGHD__IGHJ6 ${ }^{1(\mathrm{a})}$ Episodes beginning with an anisotropic (A) or mesotropic (M) SEB; ${ }^{1(b)}$ non-contributory anisotropic sub-episode block (NCA); ${ }^{1(c)}$ non-contributory single or multiple stabilizing isotropy points or reverse stabilizing isotropy point(s), NCstl; ${ }^{1(d)}$ anisotropy converted-to-mesotropy, ACM; and ${ }^{1(\mathrm{e})}$ indirect reverse stlsotropy and/or stlsotropy for anisotropy or for mesotropy, stMfA or stMfM. *ACM of initial SEB no. 5 due to 0.25 -factor-adjusted reverse stlsotropy preceding SEB (no. 5), which sums into ending confirmation mesotropic SEB no. 6, and the new ending confirmation anisotropic SEB is no. 7 (final SEB count is $6^{*}$ ). (DOC $77 \mathrm{~kb}$ ) 


\section{Abbreviations}

ACM: Anisotropy converted-to-mesotropy; esebssiwaagoT : Episodic sub-episode sums split-integrated weighted average-averaged gene overex pression tropy quotient; HR: Homologous recombination; iCSR: Internal consensus sequence recognition CSR; NCA: Non-contributory anisotropic sub-episode block; NCstl: Non-contributory single or multiple stabilizing isotropy points or reverse stabilizing isotropy point(s); $p r p T_{\mathrm{Q}}$ : Paired point tropy quotient; SEB: Sub-episode block; SHM: Somatic hypermutation; stMfA: Indirect reverse stlsotropy and/or stlsotropy for anisotropy; stMfM: Indirect reverse stlsotropy and/or stlsotropy for mesotropy

\section{Acknowledgements}

Not applicable.

\section{Funding}

No funding was applied for this research.

\section{Availability of data and materials}

The mined data utilized in this study is publicly available at the GeneCards database (https://www.genecards.org/) genomic neighborhood GeneLoc genome locator (https://genecards.weizmann.ac.il/) and the LNCipedia.org database (http://www.Incipedia.org/). All data analysed this study are included in the supplementary information files of this article.

\section{Authors' contributions}

HS conceptualized the research, developed the methodology, analyzed the data, and wrote the manuscript.

\section{Ethics approval and consent to participate} Not applicable.

\section{Consent for publication}

Not applicable.

\section{Competing interests}

The authors declare that they have no competing interests.

\section{Publisher's Note}

Springer Nature remains neutral with regard to jurisdictional claims in published maps and institutional affiliations.

\section{Received: 4 December 2017 Accepted: 26 January 2018}

\section{Published online: 02 March 2018}

\section{References}

1. Sarin H. Pressuromodulation at the cell membrane as the basis for small molecule hormone and peptide regulation of cellular and nuclear function. J Transl Med. 2015;13(372).

2. Sarin $\mathrm{H}$. Horizontal alignment of $5^{\prime}->3^{\prime}$ intergene distance segment tropy with respect to the gene as the conserved basis for DNA transcription. Future Sci OA. 2017:3(1):FSO1610.

3. Sarin H. Mechanism underlying pressuromodulation-mediated horizontal alignment of a gene for maximal transcription as predicted by the

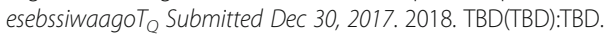

4. Sarin H. B-cell differentiation is pressuromodulated as determined by pressuromodulation mapping: Part I, cell differentiation. Transl Med Commun. In press.

5. Brinkmann V, Heusser CH. T cell-dependent differentiation of human B cells into IgM, IgG, IgA, or IgE plasma cells: high rate of antibody production by IgE plasma cells, but limited clonal expansion of IgE precursors. Cell Immunol. 1993;152(2):323-32.

6. Ho F, Lortan JE, MacLennan IC, Khan M. Distinct short-lived and long-lived antibody-producing cell populations. Eur J Immunol. 1986;16(10):1297-301.

7. Bohannon C, Powers R, Satyabhama L, Cui A, Tipton C, Michaeli M, Skountzou I, Mittler RS, Kleinstein SH, Mehr R, et al. Long-lived antigeninduced IgM plasma cells demonstrate somatic mutations and contribute to long-term protection. Nat Commun. 2016;7:11826.

8. Klein U, Kuppers R, Rajewsky K. Evidence for a large compartment of IgM-expressing memory B cells in humans. Blood. 1997;89(4):1288-98.
9. Muralidharan S, Mandrekar P. Cellular stress response and innate immune signaling: integrating pathways in host defense and inflammation. J Leukoc Biol. 2013;94(6):1167-84.

10. Dudley DD, Chaudhuri J, Bassing CH, Alt FW. Mechanism and control of V(D)J recombination versus class switch recombination: similarities and differences. Adv Immunol. 2005;86:43-112.

11. Dudley DD, Manis JP, Zarrin AA, Kaylor L, Tian M, Alt FW. Internal IgH class switch region deletions are position-independent and enhanced by AID expression. Proc Natl Acad Sci U S A. 2002:99(15):9984-9.

12. Dunnick W, Hertz GZ, Scappino L, Gritzmacher C. DNA sequences at immunoglobulin switch region recombination sites. Nucleic Acids Res. 1993 21(3):365-72.

13. Mills FC, Brooker JS, Camerini-Otero RD. Sequences of human immunoglobulin switch regions: implications for recombination and transcription. Nucleic Acids Res. 1990:18(24):7305-16.

14. White MB, Word CJ, Humphries CG, Blattner FR, Tucker PW. Immunoglobulin D switching can occur through homologous recombination in human $B$ cells. Mol Cell Biol. 1990;10(7):3690-9.

15. Alt FW, Yancopoulos GD, Blackwell TK, Wood C, Thomas E, Boss M, Coffman R, Rosenberg N, Tonegawa S, Baltimore D. Ordered rearrangement of immunoglobulin heavy chain variable region segments. EMBO J. 1984;3(6):1209-19.

16. Alt FW, Reth MG, Blackwell TK, Yancopoulos GD. Regulation of immunoglobulin variable-region gene assembly. Mt Sinai J Med. 1986;53(3):166-9.

17. Alt FW, Ferrier $P$, Malynn B, Lutzker S, Rothman P, Berman J, Blackwell K, Mellis S, Pollock R, Furley A, et al. Control of recombination events during lymphocyte differentiation. Heavy chain variable region gene assembly and heavy chain class switching. Ann N Y Acad Sci. 1988;546:9-24.

18. Arnaout R, Lee W, Cahill P, Honan T, Sparrow T, Weiand M, Nusbaum C, Rajewsky K, Koralov SB. High-resolution description of antibody heavy-chain repertoires in humans. PLoS One. 2011;6(8):e22365

19. Matsuda F, Ishii K, Bourvagnet P, Kuma K, Hayashida H, Miyata T, Honjo T. The complete nucleotide sequence of the human immunoglobulin heavy chain variable region locus. J Exp Med. 1998;188(11):2151-62.

20. Coker HA, Durham SR, Gould HJ. Local somatic hypermutation and class switch recombination in the nasal mucosa of allergic rhinitis patients. J Immunol. 2003;171(10):5602-10.

21. Shinkai Y, Koyasu S, Nakayama K, Murphy KM, Loh DY, Reinherz EL, Alt FW. Restoration of T cell development in RAG-2-deficient mice by functional TCR transgenes. Science. 1993;259(5096):822-5.

22. Hwang I, Huang JF, Kishimoto H, Brunmark A, Peterson PA, Jackson MR, Surh CD, Cai Z, Sprent J. T cells can use either T cell receptor or CD28 receptors to absorb and internalize cell surface molecules derived from antigen-presenting cells. J Exp Med. 2000;191(7):1137-48.

23. Talwar GP, Diwan M, Razvi F, Malhotra R. The impact of new technologies on vaccines. Natl Med J India. 1999;12(6):274-80

24. Takeda K, Kaisho T, Akira S. Toll-like receptors. Annu Rev Immunol. 2003;21(1):335-76

25. Kaiser DA, Sato M, Ebert RF, Pollard TD. Purification and characterization of two isoforms of Acanthamoeba profilin. J Cell Biol. 1986;102(1):221-6.

26. Proft T, Fraser JD. Bacterial superantigens. Clin Exp Immunol. 2003;133(3):299-306

27. Jeong KY, Kim C, Yong T-S. Enzymatic activities of allergen extracts from three species of dust mites and cockroaches commonly found in Korean home. The Korean Journal of Parasitology. 2010:48(2):151-5.

28. Jackson KJ, Wang Y, Collins AM. Human immunoglobulin classes and subclasses show variability in VDJ gene mutation levels. Immunol Cell Biol. 2014;92(8):729-33.

29. Xiong H, Dolpady J, Wabl M, Curotto de Lafaille MA, Lafaille JJ. Sequential class switching is required for the generation of high affinity $\lg E$ antibodies. J Exp Med. 2012;209(2):353-64.

30. Tovey ER, Chapman MD, Platts-Mills TA. Mite faeces are a major source of house dust allergens. Nature. 1981;289(5798):592-3.

31. Marsh DG, Goodfriend L, King TP, Lowenstein H, Platts-Mills TA. Allergen nomenclature. Bull World Health Organ. 1986;64(5):767-74.

32. Williams LK, Ownby DR, Maliarik MJ, Johnson CC. The role of endotoxin and its receptors in allergic disease. Annals of allergy, asthma \& immunology : official publication of the American College of Allergy, Asthma, \& Immunology. 2005;94(3):323-32.

33. Widmer F, Hayes PJ, Whittaker RG, Kumar RK Substrate preference profiles of proteases released by allergenic pollens. Clinical and experimental allergy 
journal of the British Society for Allergy and Clinical Immunology. 2000:30(4):571-6.

34. Vogel H, Jähnig F. The structure of melittin in membranes. Biophys J. 1986; 50(4):573-82.

35. Buku A. Mast cell degranulating (MCD) peptide: a prototypic peptide in allergy and inflammation. Peptides. 1999;20(3):415-20.

36. Blank S, Seismann H, Bockisch B, Braren I, Cifuentes L, Mclntyre M, Ruhl D, Ring J, Bredehorst R, Ollert MW, et al. Identification, recombinant expression, and characterization of the $100 \mathrm{kDa}$ high molecular weight hymenoptera venom allergens Api m 5 and Ves v 3. J Immunol. 2010;184(9):5403-13.

37. Bogdanov S. Bee venom: composition, health, medicine: a review. Bee Product Science; 2015. http://www.bee-hexagon.net.

38. Husebye $H$, Halaas $\varnothing$, Stenmark H, Tunheim G, Sandanger O, Bogen B, Brech A, Latz E, Espevik T: Endocytic pathways regulate Toll-like receptor 4 signaling and link innate and adaptive immunity, vol. 25; 2006.

39. Baranova IN, Vishnyakova TG, Bocharov AV, Leelahavanichkul A, Kurlander R, Chen Z, Souza AC, Yuen PS, Star RA, Csako G, et al. Class B scavenger receptor types I and II and CD36 mediate bacterial recognition and proinflammatory signaling induced by Escherichia Coli, lipopolysaccharide, and cytosolic chaperonin 60. J Immunol. 2012;188(3):1371-80.

40. Minguet S, Dopfer EP, Pollmer C, Freudenberg MA, Galanos C, Reth M, Huber M, Schamel WW. Enhanced B-cell activation mediated by TLR4 and BCR crosstalk. Eur J Immunol. 2008;38(9):2475-87.

41. Kohno M, Horibe T, Ohara K, Ito S, Kawakami K. The membrane-lytic peptides K8L9 and Melittin enter cancer cells via receptor endocytosis following subcytotoxic exposure. Chem Biol. 2014;21(11):1522-32.

42. Martín-Sánchez F, Martínez-García JJ, Muñoz-García M, Martínez-Villanueva M, Noguera-Velasco JA, Andreu D, Rivas L, Pelegrín P. Lytic cell death induced by melittin bypasses pyroptosis but induces NLRP3 inflammasome activation and IL-1 $\beta$ release. Cell Death \&Amp; Disease. 2017;8:e2984.

43. Coker HA, Harries HE, Banfield GK, Carr VA, Durham SR, Chevretton E, Hobby P, Sutton BJ, Gould HJ. Biased use of VH5 IgE-positive B cells in the nasal mucosa in allergic rhinitis. J Allergy Clin Immunol. 2005;116(2):445-52.

44. Snow RE, Chapman CJ, Frew AJ, Holgate ST, Stevenson FK. Pattern of usage and somatic hypermutation in the $\mathrm{V}(\mathrm{H}) 5$ gene segments of a patient with asthma: implications for IgE. Eur J Immunol. 1997;27(1):162-70.

45. Rogosch T, Kerzel S, Dey F, Wagner JJ, Zhang Z, Maier RF, Zemlin M. IgG4 and IgE transcripts in childhood allergic asthma reflect divergent antigendriven selection. J Immunol. 2014;193(12):5801-8.

46. Varga EM, Kausar F, Aberer W, Zach M, Eber E, Durham SR, Shamji MH. Tolerant beekeepers display venom-specific functional lgG4 antibodies in the absence of specific lgE. J Allergy Clin Immunol. 2013;131(5):1419-21.

47. Matysiak J, Matysiak J, Bręborowicz A, Kycler Z, Dereziński P, Kokot Z. Immune and clinical response to honeybee venom in beekeepers, vol. 23 . 2016.

\section{Submit your next manuscript to BioMed Central and we will help you at every step:}

- We accept pre-submission inquiries

- Our selector tool helps you to find the most relevant journal

- We provide round the clock customer support

- Convenient online submission

- Thorough peer review

- Inclusion in PubMed and all major indexing services

- Maximum visibility for your research

Submit your manuscript at www.biomedcentral.com/submit

) Biomed Central 